\title{
Scattering amplitudes and BCFW recursion in twistor space
}

\author{
Lionel Mason and David Skinner \\ The Mathematical Institute, University of Oxford, \\ 24-29 St. Giles, Oxford OX1 3LP, U.K. \\ Institut des Hautes Études Scientifiques, Le Bois Marie, \\ 35, Route de Chartres, 91440 Bures-sur-Yvette, France \\ E-mail: lmason@maths.ox.ac.uk, dskinner@perimeterinstitute.ca
}

ABSTRACT: Twistor ideas have led to a number of recent advances in our understanding of scattering amplitudes. Much of this work has been indirect, determining the twistor space support of scattering amplitudes by examining the amplitudes in momentum space. In this paper, we construct the actual twistor scattering amplitudes themselves. We show that the recursion relations of Britto, Cachazo, Feng and Witten have a natural twistor formulation that, together with the three-point seed amplitudes, allows us to recursively construct general tree amplitudes in twistor space. We obtain explicit formulae for $n$ particle MHV and NMHV super-amplitudes, their CPT conjugates (whose representations are distinct in our chiral framework), and the eight particle $\mathrm{N}^{2} \mathrm{MHV}$ super-amplitude. We also give simple closed form formulae for the $\mathcal{N}=8$ supergravity recursion and the MHV and $\overline{\mathrm{MHV}}$ amplitudes. This gives a formulation of scattering amplitudes in maximally supersymmetric theories in which superconformal symmetry and its breaking is manifest.

For $\mathrm{N}^{k} \mathrm{MHV}$, the amplitudes are given by $2 n-4$ integrals in the form of Hilbert transforms of a product of $n-k-2$ purely geometric, superconformally invariant twistor delta functions, dressed by certain sign operators. These sign operators subtly violate conformal invariance, even for tree-level amplitudes in $\mathcal{N}=4$ super Yang-Mills, and we trace their origin to a topological property of split signature space-time. We develop the twistor transform to relate our work to the ambidextrous twistor diagram approach of Hodges and of Arkani-Hamed, Cachazo, Cheung and Kaplan.

KEYwords: Supersymmetric gauge theory, Duality in Gauge Field Theories

ArXiv ePrint: 0903.2083 


\section{Contents}

1 Introduction 1

2 The momentum shift on twistor space $\quad 6$

3 Twistor amplitudes and conformal invariance $\quad 7$

$\begin{array}{lll}3.1 & \text { Distributions on projective twistor space } & 8\end{array}$

3.1.1 The Hilbert transform and the sgn function 11

$\begin{array}{ll}3.2 \text { Seed amplitudes in } \mathcal{N}=4 \mathrm{SYM} & 12\end{array}$

$\begin{array}{lll}3.3 & \text { On conformal invariance } & 14\end{array}$

4 BCFW recursion in twistor space $\quad 16$

$\begin{array}{ll}4.1 \text { The } \mathrm{i} \epsilon \text {-prescription on } \mathbb{R}^{2,2} & 18\end{array}$

$\begin{array}{lll}4.2 & \text { Transform to twistor space } & 19\end{array}$

5 SYM twistor amplitudes from BCFW recursion 21

5.1 On the general structure of $\mathcal{N}=4$ amplitudes $\quad 21$

$\begin{array}{ll}5.2 & \text { Solving the recursion relations } \\ 5.2 .1 & 22\end{array}$

5.2.1 The homogeneous term and MHV amplitudes 23

5.2.2 The conjugate homogeneous term and the $\overline{\mathrm{MHV}}$ amplitudes 24

$\begin{array}{lll}5.3 & \text { NMHV amplitudes } & 25\end{array}$

$\begin{array}{ll}5.4 & \text { Conjugate NMHV amplitudes }\end{array}$

5.5 Further tree examples 28

$\begin{array}{lll}5.6 & \text { General tree amplitudes } & 29\end{array}$

$\begin{array}{lll}5.7 & \text { Some elementary loop amplitudes } & 30\end{array}$

$6 \quad$ Twistor supergravity amplitudes 33

6.1 The homogeneous term and the MHV amplitudes 34

6.2 The conjugate homogeneous term and $\overline{\mathrm{MHV}}$ amplitudes 35

$\begin{array}{lll}7 & \text { An ambidextrous approach } & \mathbf{3 5}\end{array}$

$\begin{array}{lll}7.1 & \text { Fourier transforms and twistor transforms } & 36\end{array}$

$\begin{array}{lll}\text { 7.1.1 Supersymmetric twistor transforms } & 38\end{array}$

$\begin{array}{lll}7.2 & \text { The inner product } & 38\end{array}$

$\begin{array}{lll}\text { 7.3 } & \text { BCFW recursion in ambitwistor space } & 39\end{array}$

7.4 Ambidextrous form of the 3 point amplitudes 41

8 Conclusions and outlook $\quad 42$

$\begin{array}{lll}8.1 & \text { Signature signs and loops } & 45\end{array}$

$\begin{array}{ll}\text { A Conventions, notation and background } & 47\end{array}$ 
B The X-ray and half Fourier transforms

B.1 Scalar fields 49

B.2 Generalisation to other helicities 51

B.3 Supersymmetry 52

B.3.1 Superfields in twistor space and on-shell momentum space $\quad 53$

C The half Fourier transform of seed amplitudes $\quad 55$

$\begin{array}{lll}\text { C.1 The 3-point MHV amplitude } & 55\end{array}$

$\begin{array}{ll}\text { C.2 The 3-point } \overline{\mathrm{MHV}} \text { amplitude } & 57\end{array}$

$\begin{array}{lll}\text { C.3 The } n \text {-point MHV amplitude } & 58\end{array}$

\section{Introduction}

In his development of twistor-string theory [1], Witten showed that gauge theory scattering amplitudes have remarkable properties when analyzed in twistor space. The subsequent activity led to substantial progress in our understanding of amplitudes including the construction of the tree-level Yang-Mills S-matrix via the connected prescription of twistorstrings [2], the MHV diagram formalism [3], the BCFW recursion relations [4, 5] and the generalized unitarity and leading singularity methods [6-14]. Despite there having been much work examining the support of amplitudes in twistor space (see e.g. [1, 3, 15-21]), until this point there has been no systematic study of explicit formulae for the actual twistor space amplitudes themselves.

There are many reasons why it is interesting to investigate the twistor representation of scattering amplitudes more closely. Firstly, such an analysis should make the conformal properties of scattering amplitudes manifest; these can be difficult to see on momentum space. Secondly, twistor theory brings out the complete integrability of the anti self-dual sectors of both Yang-Mills and General Relativity [22-25]; in the language of twistor actions [26-29], there exist gauges for which the anti-selfdual sector is free [30]. Furthermore, Drummond, Henn \& Plefka [31] have recently shown that the generators of the dual superconformal algebra $[32,33]$ - one of the main tools in the construction of multi-particle [34, 35] and multi-loop [7-9, 14, 36, 37] scattering amplitudes in $\mathcal{N}=4 \mathrm{SYM}$ - have a simple (though second-order) representation on twistor space.

Additional motivation comes from Penrose's twistor programme [38], which seeks to reformulate fundamental physics on twistor space as a route towards quantum gravity. Indeed, there has been a long-standing programme to understand scattering amplitudes in twistor theory via twistor diagrams [39, 40], but this has proved technically difficult for two reasons. Firstly, the standard form of the Penrose transform of on-shell states requires the use of cohomology: this builds in extra gauge freedom, and requires one to understand multi-dimensional contour integrals in large complex manifolds. In this paper, cohomology will be sidestepped at the expense of working in $(++--)$ space-time signature. With this signature, twistor space has a real slice $\mathbb{R P}^{3}$ and massless fields on split signature space-time 
correspond to homogenous functions on real twistor space, rather than cohomology classes. The Penrose transform then becomes the closely related 'X-ray transform' of Fritz John [41] (see also [42]) and, as we show in appendix B, the Fourier transform of the X-ray transform is precisely Witten's half Fourier transform [1] to on-shell momentum space. We will base our analysis on this half Fourier transform. The expectation is that the twistorial structures underlying amplitudes in all signatures will be visible in this signature also, although we will see that complications that seem to be specific to split signature also arise in this approach.

A second technical problem one encounters when trying to describe twistor scattering amplitudes is that off-shell states (arising in the internal legs of a Feynman diagram) cannot be encoded holomorphically on twistor space alone. In momentum space, such off-shell states are easily incorporated by relaxing the condition that the wavefunction is supported only on the mass shell. In the twistor diagram approach [39, 40], one instead relaxes the condition that the wavefunction be holomorphic, and then proceeds to complexify $f(Z, \bar{Z}) \rightarrow f(Z, W)$. This procedure leads to a description in terms of both ${ }^{1}$ twistors and dual twistors and hence provides a six-dimensional description of four-dimensional objects, implying still more redundancy. However, the recursion relations discovered by Britto, Cachazo \& Feng [4] and proved by Britto, Cachazo, Feng \& Witten [5] involve only on-shell, gauge invariant objects, thereby eliminating much of the redundancy (even on momentum space) of the Feynman diagram approach to scattering amplitudes. The BCFW relations generate the full perturbative content of Yang-Mills and gravity starting from only the three-particle amplitudes for $\langle++-\rangle$ and $\langle--+\rangle$ helicity configurations. Basing a twistor scattering theory on the BCFW relations allows us to avoid this second difficulty.

The first twistor formulation of the $\mathrm{BCFW}$ recursion procedure was given in terms of twistor diagrams by Hodges [43-45]. Hodges' construction uses both twistors and dual twistors, and has recently been re-derived by Arkani-Hamed, Cachazo, Cheung \& Kaplan [46] in work that is parallel to this paper, and similarly uses Witten's half Fourier transform. By contrast, in this article we will work with a chiral formulation in which all external states are represented on $\left(\mathrm{dual}^{2}\right)$ twistor space. The twistor diagram representation of [43-46] is related to ours by a (complete) Fourier transform on non-projective twistor space. In section 7 we discuss the relation of this Fourier transform to the twistor transform between projective twistor and dual twistor spaces, and use this to explain the detailed relation between the work of Arkani-Hamed et al. [46] and the current paper.

If only dual twistor variables $\mathrm{W}$ are used, the (super-)momentum shift that is the first step in the BCFW procedure corresponds to the simple shift

$$
\mathcal{A}\left(\mathrm{W}_{1}, \ldots, \mathrm{W}_{n}\right) \rightarrow \mathcal{A}\left(\mathrm{W}_{1}, \ldots, \mathrm{W}_{n}-t \mathrm{~W}_{1}\right)
$$

\footnotetext{
${ }^{1}$ Twistor diagrams are ambidextrous, and one must choose arbitrarily whether to represent a given external state on twistor or dual twistor space. While there is no problem with working with these two representations simultaneously (analogous to describing some particles on momentum space and others on space-time), for a basic description in terms of an action, one needs to specify the basic fields and the space on which they live.

${ }^{2}$ For better agreement with the perturbative scattering theory literature, we work on dual twistor space, where Yang-Mills amplitudes with 2 negative and $n-2$ positive helicity gluons are supported on a line. A full summary of our conventions may be found in appendix A.
} 
in the twistor amplitude $\mathcal{A}$, where $t$ is the shift parameter. This formula is proved in section 2 and makes manifest the (super-)conformal invariance of the BCFW shift. The original use of the shift was to introduce a complex parameter in which propagators within the amplitude generate poles, so that contour integration yields the recursion relation as a residue formula. Here, our aim is not to prove the recursion relation (for which see $[5,34,47,48])$. Instead, $t$ will be a real parameter that provides the one degree of freedom required to go off-shell in twistor space. This is the key idea from the point of view of a twistor theorist seeking to study perturbative scattering theory: rather than representing an off-shell state by both a twistor and a dual twistor as in twistor diagrams, one can describe it using a single twistor together with a BCFW shift. (See also [49] for a hybrid formulation in which off-shell states are treated on momentum space while on-shell states are treated on twistor space.)

To begin the recursion procedure, one must seed the BCFW relations with the threeparticle amplitudes. In $(++--)$ space-time, these can be obtained directly by taking the half Fourier transform of the momentum space expressions. Doing so leads in the first instance to formulae ${ }^{3}$ whose superconformal properties remain somewhat obscure, in contrast to our aim of making such behaviour transparent. To remedy this, in section 3.1 we introduce distributions on twistor space that are manifestly conformally invariant. These are the basic objects out of which we construct the seed amplitudes in section 3.2.

One of the surprises of our analysis is that these basic three-particle amplitudes are not conformally invariant, even in $\mathcal{N}=4 \mathrm{SYM}$. The failure of conformal invariance is rather subtle and is discussed in section 3.3 and further in the conclusions. In some sense, it is merely the problem of choosing an overall sign for the amplitude; nevertheless, there is a topological obstruction to doing this in a way that is consistent with conformal invariance. The obstruction is analogous to the impossibility of choosing a holomorphic branch for $\sqrt{z}$ on the punctured complex plane; one must choose a cut across which the function will not be holomorphic. Similarly, to make the sign factor in the amplitude well-defined requires the choice of a light-cone at infinity. Moreover, although the BCFW shift is superconformally invariant, the recursion relations themselves are not. Once again, the violation of conformal invariance is rather subtle; for example we explicitly show that when $n$ is odd, the $n$-particle MHV and googly MHV super-amplitudes in $\mathcal{N}=4$ SYM break conformal invariance even at tree-level - in the same way as the three-particle amplitudes. However, when $n$ is even the conformal breaking of the seed amplitudes and the recursion relations cancel each other out, so that these MHV (and hence googly MHV) amplitudes are genuinely conformally invariant. We argue in the conclusion that the origin of the failure of conformal invariance in our formalism is likely to be associated with our resorting to split signature in order to side-step cohomology. The topological obstruction is only present in split signature. Furthermore, in the twistor actions for gauge theory $[1,26,27]$ the relevant sign factors are essentially determined by the differential forms used in the Dolbeault cohomology description, but these signs are lost when reducing the forms to functions so as to reach a split signature description.

\footnotetext{
${ }^{3}$ See appendix $\mathrm{C}$ for a detailed derivation of the half Fourier transforms of various momentum space amplitudes
} 
In section 4 we translate the BCFW recursion relation itself onto twistor space, obtaining a recursion formula that decomposes arbitrary tree-level twistor amplitudes into more elementary ones. We focus on maximally supersymmetric Yang-Mills and gravity, and so only discuss the twistor form of the supersymmetric BCFW relations [34, 48]. Despite their extra field content, these theories are much simpler than their non-supersymmetric counterparts, even at tree-level (where the non-supersymmetric theories are contained as a subset). This is because there are $2^{n} n$-particle scattering amplitudes in non-supersymmetric YangMills or gravity, corresponding to the different choices of helicity for the external particles, whereas there are only $(n+1)$-distinct $n$-particle tree amplitudes in $\mathcal{N}=4 \mathrm{SYM}$ or $\mathcal{N}=8$ $\mathrm{SG}$ - the $\mathrm{N}^{k} \mathrm{MHV}$ amplitudes, associated with a polynomial of degree $(k+2) \mathcal{N}$ in the on-shell Grassmann momenta. This exponential simplification was a crucial ingredient in the recent solutions of the classical Yang-Mills S-matrix by Drummond \& Henn [35], and the classical gravitational S-matrix by Drummond, Spradlin, Volovich \& Wen [53] using the supersymmetric version of BCFW recursion.

Armed with the twistor form of both the seed amplitudes and the BCFW recursion relation, in section 5 we proceed to construct twistor space versions of various tree amplitudes in $\mathcal{N}=4 \mathrm{SYM}$. A simple consequence of the twistor representation of the recursion relations and the tree amplitudes is that, modulo some sign factors, the $\mathrm{N}^{k} \mathrm{MHV}$ components of the complete classical S-matrix of $\mathcal{N}=4 \mathrm{SYM}$ can written as an integral over $2 n-4$ 'shift' parameters of $n-k+2$ super-conformally invariant delta functions $\delta^{4 \mid 4}(W)$. In more detail, when an amplitude is expressed via recursion as a combination of two subamplitudes with one being a three-point MHV or $\overline{\mathrm{MHV}}$ amplitude, the integrals can be performed explicitly leading to an action of operators $\mathcal{H}_{i-1, i+1}^{i} \delta^{4 \mid 4}\left(W_{i}\right)$ or $\widetilde{\mathcal{H}}_{i}^{i-1, i+1}$ respectively. These insert a new particle at point $i$ in between particles $i-1$ and $i+1$ in the other subamplitude, the first preserving MHV degree and the second raising it. The $\mathcal{H}$ and $\widetilde{\mathcal{H}}$ operators are each a pair of integral operators (Hilbert transforms) integrating shift parameters in the amplitudes on which they act. These Hilbert transforms can formally be expressed as the sign functions of certain first order differential operators. These two operations seem to be sufficient to generate the general amplitude (at least as far as the examples we have calculated are concerned) as a sum of terms consisting of $n-3$ such operators acting on a three point amplitude. These two operators can be identified with the inverse soft limits of [46]. We explicitly perform the recursion for the $n$-particle MHV and NMHV super-amplitudes, their CPT conjugates, and the 8-particle $\mathrm{N}^{2} \mathrm{MHV}$ superamplitude. We also give an algorithm for computing some more general tree amplitude from the BCFW recursion relations.

We consider $\mathcal{N}=8$ supergravity in section 6 . The structure of the BCFW recursion relation is unchanged compared to Yang-Mills except that the sum is over all partitions, the seed amplitudes are different and we work with $\mathcal{N}=8$. We solve the recurrence in this case to give the MHV and $\overline{\mathrm{MHV}}$ amplitudes. For gravity the general shape of the result is essentially the same as for Yang-Mills (i.e., $2 n-4$ bosonic integrals of $n-k-2$ delta functions) except that certain derivatives and additional factors are introduced at each stage. A partial solution of the BCFW recursion for gravity has recently been constructed in [53], using the interplay of the KLT relations [62] (which motivate a particular form 
for the MHV amplitude [63]) with the properties of the earlier solution of the $\mathcal{N}=4$ SYM classical S-matrix [35]. It is clear that there is a very close relationship between the twistor $\mathcal{N}=8 \mathrm{SG}$ and $\mathcal{N}=4 \mathrm{SYM}$ amplitudes for the twistor amplitudes also in the sense that the underlying structure of the Hilbert transforms and corresponding support of the amplitudes is the same in both cases. The distinction between the two sets of amplitudes that there are extra factors and derivatives in the Gravitational case.

We go on to give a preliminary discussion of loops. We first give the half Fourier transform of the dimensionally regularised 4 particle 1-loop amplitude. The analytic continuation of such an amplitude to split signature is ambiguous as, for example, we are taking logs or fractional powers of quantities that have a definite sign in Lorentz signature, but which have no fixed sign in split signature. We can nevertheless find formulae for both the finite and IR divergent parts of the amplitude by choosing an analytic continuation to split signature and performing the half-Fourier transform. We show that gives a straightforward answer although of course it depends on the choice of analytic continuation we started with. We discover that the finite part is superconformally invariant. The non-supersymmetric (finite) all plus loop amplitude is rather easier as it is a rational function on momentum space with unique analytic continuation, and we give this also. It is also to be hoped that the generalised unitarity and leading singularity methods [6-14] have a natural formulation on twistor space, ${ }^{4}$ there is no analytic continuation issue for leading singularities.

We do of course intend that eventually there will be a systematic method for obtaining loop amplitudes on twistor space. We give a further discussion on the prosepects for this in section 8.1 in the conclusions. The main conclusion is that in order to translate from the formalism obtained here to one appropriate to Lorentz signature, we must re-interpret our twistor functions as representatives of Cech cohomology classes, and re-interpret all our integrals as contour integrals. In that re-interpretation, the sign factors can be dropped from the formulae, but must then be incorporated as part of the definition of the Cech cocycles dictating which sets in the cover the Cech cocycles should be attached to.

The twistor space support of amplitudes has previously been analysed and fruitfully exploited by a number of authors [1, 3, 15-21]. However, this was done implicitly, e.g. by use of differential operators acting on the momentum space amplitudes, or by integral representations. Our explicit representation of the twistor amplitudes is in fact smeared out by certain non-local operators that also break conformal symmetry. It is reasonable to regard these as an artifact of the use of split signature ${ }^{5}$ and it is therefore reasonable to ignore them if one is interested in the structures valid in the complex or other signatures; this is also the conclusion of the discussion in section 8.1. If the non-local sign operators are ignored, we find that the MHV amplitudes are indeed localized along lines (indeed they are simply products of delta-functions that restrict the corresponding twistors to lines). Howver, the NMHV amplitudes are a sum of terms that are supported on three lines in

\footnotetext{
${ }^{4}$ See $[54,56-59]$ for subsequent work that realizes this aim.

${ }^{5}$ These operators are signs on momentum space, and so are not visible to the differential operators that were used there to determine the twistor support of the amplitudes. Similarly, they are not visible when momentum space conformal generators are used to test for conformal invariance, unless the detailed behaviour of the amplitudes across its singularities is examined.
} 
contradiction to the expectations raised by the MHV formalism [1, 3, 15-21]. It is clear that some decomposition and re-summation is needed for agreement with the expectations of the MHV formalism. However, this picture is in agreement with the NMHV 1-loop formulae [51]. At higher $\mathrm{N}^{k} \mathrm{MHV}$ degree, the Hilbert transforms lead to an additional two lines for each $k$. Subsequent to version one of this paper, this picture has been re-expressed more concretely and much extended [52, 58].

At present, although the twistor form of the BCFW recursion relation and scattering amplitudes have many remarkable properties, this work does not constitute a complete theory in twistor space, because both the BCFW relation itself and the three-point seed amplitudes currently need to be imported from momentum space by half Fourier transform. We conclude in section 8 with a discussion of a possible way to relate our results to the twistor action of [27] (and its ambitwistor cousin [64]), which goes some way towards making the twistor theory self-contained. Our approach is complementary to the momentum space picture Drummond \& Henn [35], and we discuss the relation of the dual superconformal invariants of $[14,33,35]$ to the twistor space structures we find. ${ }^{6}$

Note that one can similarly transform Risager's momentum shift [65] and its multiline extensions $[66,67]$ into twistor space, obtaining a twistor space version of the MHV diagram formalism.

In appendix A we summarise our conventions and the basic background structures and formulae. In appendix B we derive the half Fourier transform from the X-ray transform. In appendix $\mathrm{C}$ we give the basic calculations of the half Fourier transform of the seed amplitudes.

\section{The momentum shift on twistor space}

The amplitude $A(1, \ldots, n)$ for a process with $n$ massless particles is a function of $n$ null momenta $p_{1}, \ldots, p_{n}$. Decomposing these null momenta into their spinor factors $\left.p_{i}=\mid i\right]\langle i|$ (where $\mid i]$ and $|i\rangle$ are spinor-helicity notation for left and right spinors $\tilde{\lambda}_{i A^{\prime}}$ and $\lambda_{i A}$, respectively) the BCFW procedure starts by shifting two of them:

$$
\mid i] \rightarrow \mid \hat{i}]:=\mid i]+t \mid j], \quad|j\rangle \rightarrow|\hat{j}\rangle:=|j\rangle-t|i\rangle .
$$

This shift apparently treats left- and right-handed spinors symmetrically. However, there is some chirality in the BCFW shift (2.1) because the 'permissible shifts' - whether one should translate a given state's primed spinor or unprimed spinor - are correlated with the helicities of the states being shifted [5]. This chirality is more apparent in the maximally supersymmetric extensions of the BCFW procedure [34, 48]: any (irreducible) representation of an $\mathcal{N}=4$ YM supermultiplet or an $\mathcal{N}=8$ gravity supermultiplet with maximal on-shell supersymmetry is necessarily chiral, as either the positive or negative helicity state must be chosen to sit at the top of the supermultiplet. In particular, all external supermultiplets have the same helicity, so (2.1) together with the 'permissible shift' rule are replaced by

\footnotetext{
${ }^{6}$ Subsequent to version 1 of this paper, the relationships betweeen twistor amplitudes and dual conformal invariance are now much better understood [55-57]
} 
the chiral super-shift

$$
\|i \rrbracket \rightarrow\| \hat{i} \rrbracket:=\|i \rrbracket+t\| j \rrbracket, \quad|j\rangle \rightarrow|\hat{j}\rangle:=|j\rangle-t|i\rangle,
$$

where $\| i \rrbracket=\left(\tilde{\lambda}_{i}, \eta_{i}\right)$ denotes both the primed spinor momenta and the Grassmann coordinate of the on-shell momentum superspace of the $i^{\text {th }}$ state; $\eta_{j}$ itself is not shifted.

In $(++--)$ space-time signature, Witten showed in [1] that the (dual) twistor and on-shell momentum space superfields are related by the half Fourier transforms

$$
f(\lambda, \mu, \chi)=\int \mathrm{d}^{2 \mid \mathcal{N}} \tilde{\lambda} \mathrm{e}^{\mathrm{i} \llbracket \mu \tilde{\lambda} \rrbracket} \Phi(\lambda, \tilde{\lambda}, \eta) ; \quad \Phi(\lambda, \tilde{\lambda}, \eta)=\frac{1}{(2 \pi)^{2}} \int \mathrm{d}^{2} \mu \mathrm{e}^{-\mathrm{i} \llbracket \mu \tilde{\lambda} \rrbracket} f(\mu, \lambda, \chi),
$$

where $\left(\lambda_{A}, \mu^{A^{\prime}}\right)$ and $\chi_{a}$ are the bosonic and fermionic components of a (dual) supertwistor $\mathrm{W}$, and

$$
\llbracket \mu \tilde{\lambda} \rrbracket:=\mu^{A^{\prime}} \tilde{\lambda}_{A^{\prime}}+\chi_{a} \eta^{a}
$$

is the natural pairing between $(\mu, \chi)$ and the on-shell momentum space co-ordinates $(\tilde{\lambda}, \eta)$. Under the momentum supershift (2.2) (choosing $i=1$ and $j=n$ for simplicity), the twistor super-amplitude transforms as

$$
\begin{aligned}
\widehat{\mathcal{A}}\left(\mathrm{W}_{1}, \ldots, \mathrm{W}_{n}\right) & =\int \prod_{i=1}^{n} \mathrm{~d}^{2 \mid \mathcal{N}} \tilde{\lambda}_{i} \mathrm{e}^{\mathrm{i} \llbracket \mu_{i} \tilde{\lambda}_{i} \rrbracket} A(\hat{1}, \ldots, \hat{n}) \\
& =\int \mathrm{d}^{2 \mid \mathcal{N}} \hat{\tilde{\lambda}}_{1} \mathrm{~d}^{2 \mid \mathcal{N}} \tilde{\lambda}_{n} \mathrm{e}^{\mathrm{i} \llbracket \mu_{1} \hat{\tilde{\lambda}}_{1} \rrbracket} \mathrm{e}^{\mathrm{i} \llbracket\left(\mu_{n}-t \mu_{1}\right) \tilde{\lambda}_{n} \rrbracket} \prod_{j=2}^{n-1} \mathrm{~d}^{2 \mid \mathcal{N}} \tilde{\lambda}_{j} \mathrm{e}^{\mathrm{i} \llbracket \mu_{j} \tilde{\lambda}_{j} \rrbracket} A(\hat{1}, \ldots, \hat{n}) \\
& =\mathcal{A}\left(\mathrm{W}_{1}, \ldots, \mathrm{W}_{n}-t \mathrm{~W}_{1}\right) ;
\end{aligned}
$$

i.e. the half Fourier transform combines with the shift $|n\rangle \rightarrow|n\rangle-t|1\rangle$ in the unprimed spinor to result in a shift of the entire (super)twistor ${ }^{7} \mathrm{~W}_{n}$ along the line joining it to $\mathrm{W}_{1}$. Equation (2.5) provides a key motivation for the rest of this paper. It shows that the BCFW shift is (super)conformally invariant and may be simply expressed on twistor space.

\section{Twistor amplitudes and conformal invariance}

The BCFW recursion procedure is seeded by the three-point MHV and $\overline{\mathrm{MHV}}$ amplitudes. For $\mathcal{N}=4 \mathrm{SYM}$, twistor space versions of these may be obtained by directly taking the half Fourier transform of the momentum space expressions

$$
\begin{aligned}
A_{\mathrm{MHV}}(1,2,3) & =\frac{\delta^{(4 \mid 8)}\left(\sum_{i=1}^{3}|i\rangle \llbracket i \|\right)}{\langle 12\rangle\langle 23\rangle\langle 31\rangle} \\
A_{\overline{\mathrm{MHV}}}(1,2,3) & =\frac{\delta^{(4)}\left(\sum_{i=1}^{3}|i\rangle[i \mid) \delta^{(0 \mid 4)}\left(\eta_{1}[23]+\eta_{2}[31]+\eta_{3}[12]\right)\right.}{[12][23][31]} .
\end{aligned}
$$

${ }^{7}$ That only $\mathrm{W}_{n}$ is shifted should not be surprising: (2.2) is generated by $\tilde{\lambda}_{n} \partial / \partial \tilde{\lambda}_{1}, \eta_{n} \partial / \partial \eta_{1}$ and $-\lambda_{1} \partial / \partial \lambda_{n}$. Under the half Fourier transform (2.3) one replaces $\tilde{\lambda} \rightarrow \partial / \partial \mu, \partial / \partial \tilde{\lambda} \rightarrow-\mu, \eta \rightarrow \partial / \partial \chi$ and $\partial / \partial \eta \rightarrow-\chi$, so these generators combine to form $-\mathrm{W}_{1} \partial_{\mathrm{W}_{n}}$. 
For example, for the 3-particle MHV amplitude one finds in the first instance ${ }^{8}$

$$
\mathcal{A}_{\mathrm{MHV}}\left(\mathrm{W}_{1}, \mathrm{~W}_{2}, \mathrm{~W}_{3}\right)=\frac{\delta^{(2 \mid 4)}\left(\mu_{1}\langle 23\rangle+\mu_{2}\langle 31\rangle+\mu_{3}\langle 12\rangle\right)}{\langle 12\rangle\langle 23\rangle\langle 31\rangle},
$$

where the $\delta$-functions run over the supertwistor components $\left(\mu^{A^{\prime}}, \chi_{a}\right) . \mathcal{A}_{\mathrm{MHV}}\left(\mathrm{W}_{1}, \mathrm{~W}_{2}, \mathrm{~W}_{3}\right)$ has support precisely where $\mathrm{W}_{1}, \mathrm{~W}_{2}$ and $\mathrm{W}_{3}$ are collinear, and has the standard 'current correlator' denominator [68] that inspired twistor-string theory [1].

While (3.2) is manifestly (super)Poincaré invariant, its conformal properties are still not transparent, since $\left(\mu^{A^{\prime}}, \chi_{a}\right)$ appear in (3.2) on a rather different footing to $\lambda_{A}$. As indicated in the introduction, the conformal properties of scattering amplitudes are exhibited most clearly by writing them in terms of manifestly $\operatorname{SL}(4 \mid \mathcal{N} ; \mathbb{R})$ invariant $^{9}$ distributions on real projective twistor space. In section 3.1 we discuss the twistor distributions that in section 3.2 turn out to be relevant for describing twistor space scattering amplitudes.

\subsection{Distributions on projective twistor space}

The most elementary distribution is the delta function supported at a point $Y \in \mathbb{R} \mathbb{P}^{3}$, We write this as $\delta_{-n-4}^{(3)}(W, Y)$ and it has the defining property

$$
f(Y)=\int_{\mathbb{R}^{3}} f(W) \delta_{-n-4}^{(3)}(W, Y) D^{3} W
$$

for $f$ a function of homogeneity degree $n$ and where $D^{3} W:=\frac{1}{4} \epsilon^{\alpha \beta \gamma \delta} W_{\alpha} \mathrm{d} W_{\beta} \wedge \mathrm{d} W_{\gamma} \wedge \mathrm{d} W_{\delta}$ is the canonical top form of homogeneity +4 . This $\mathbb{R P}^{3}$ delta function can be described using an elementary integral of the standard, non-projective $\delta$-function on $\mathbb{R}^{4}$ :

$$
\delta_{-n-4}^{(3)}(W, Y)=\int_{-\infty}^{\infty} \frac{\mathrm{d} t}{t^{1+n}} \operatorname{sgn}(t) \delta^{(4)}(W-t Y) .
$$

Equation (3.4) has the right support because $W_{\alpha}-t Y_{\alpha}=0$ only if $W$ and $Y$ lie on the same line through the origin in $\mathbb{R}^{4}$ and hence define the same point in the projective space. It is also easy to check that under the scalings $W_{\alpha} \rightarrow a W_{\alpha}$ and $Y_{\alpha} \rightarrow b Y_{\alpha}$, we have (at least for $a / b>0$ )

$$
\delta_{-n-4}^{(3)}(a W, b Y)=\frac{b^{n}}{a^{n+4}} \delta_{-n-4}^{(3)}(W, Y),
$$

so that the subscript labels the homogeneity of the first entry. As $\mathbb{R}-\{0\}$ is not connected, the behaviour under sign reversal must be considered separately. Scaling $\left(W_{\alpha}, Y_{\alpha}\right) \rightarrow$ $\left(a W_{\alpha}, b Y_{\alpha}\right)$ with $a / b<0$ induces the scaling

$$
\frac{\mathrm{d} t}{t^{1+n}} \operatorname{sgn}(t) \rightarrow(-1) \times \frac{b^{n}}{a^{n}} \frac{\mathrm{d} t}{t^{1+n}} \operatorname{sgn}(t)
$$

where the extra sign change comes from the factor of $\operatorname{sgn}(t)$. However, under this scaling the limits of the $t$ integral also change sign, so that $\delta_{-n-4}^{(3)}(W, Y)$ itself has no extra signs. These properties ensure that (3.3) is well-defined projectively whenever $f(W)$ is.

\footnotetext{
${ }^{8}$ See appendix $\mathrm{C}$ for a detailed derivation of the half Fourier transforms of various momentum space amplitudes.

${ }^{9}$ The superconformal group in $(++--)$ signature space-time is $\operatorname{SL}(4 \mid \mathcal{N} ; \mathbb{R})$ or $\operatorname{PSL}(4 \mid 4 ; \mathbb{R})$ when $\mathcal{N}=4$.
} 
Perhaps surprisingly, we actually do want to consider twistor functions and distributions that have wrong sign behaviour, in the sense that

$$
\tilde{f}(a W)= \begin{cases}a^{n} \tilde{f}(W) & a \in \mathbb{R}^{+} \\ -a^{n} \tilde{f}(W) & a \in \mathbb{R}^{-}\end{cases}
$$

so that they scale with an extra minus sign when the scaling parameter is negative. ${ }^{10}$ For these we can define a tilded $\delta$-function $\tilde{\delta}_{-n-4}^{(3)}(W, Y)$ such that

$$
\tilde{f}(Y)=\int_{\mathbb{R P}^{3}} \tilde{f}(W) \tilde{\delta}_{-n-4}^{(3)}(W, Y) D^{3} W
$$

For (3.8) to be well-defined, $\tilde{\delta}_{-n-4}^{(3)}(W, Y)$ must have 'wrong sign' behaviour in both $W$ and $Y$, so it is related to (3.4) by

$$
\tilde{\delta}_{-n-4}^{(3)}(W, Y)=\operatorname{sgn}\left(\frac{W}{Y}\right) \delta_{-n-4}^{(3)}(W, Y)=\int_{-\infty}^{\infty} \frac{\mathrm{d} t}{t^{1+n}} \delta^{(4)}(W-t Y),
$$

where the second equality follows because $t=W / Y$ on the support of the $\delta$-function.

In concrete calculations, the $\mathrm{d} t$ integrals are easily performed explicitly using one of the $\delta$-functions, say the component of the $|\lambda\rangle$ spinor in the direction of some fixed spinor $|\alpha\rangle$. On the support of $\delta^{(4)}\left(W_{1}-t W_{2}\right), t=\langle\alpha 1\rangle /\langle\alpha 2\rangle$, so we can reduce to three $\delta$-functions. However this breaks conformal invariance. Both the $\delta^{(3)}(W, Y)$ and $\tilde{\delta}^{(3)}(W, Y)$ enforce the conformally invariant condition that $W$ and $Y$ coincide projectively, but the only ways for us to express this condition invariantly is via the formal definition (3.3) or the integral formulae (3.4) \& (3.9). This is because it is not possible to impose $W_{\alpha} \propto Y_{\alpha}$ with an invariant set of irreducible equations: The three conditions $W_{\alpha} \propto Y_{\alpha}$ are given by the six equations

$$
W_{\alpha} Y_{\beta}-W_{\beta} Y_{\alpha}=0
$$

but are subject to three relations. Choosing any three of the equations breaks conformal invariance and will also admit spurious solutions for which $W_{\alpha}$ is not proportional to $Y_{\alpha}$. The integrals over $\mathrm{d} t$ above are the easiest way to express the full projective invariance.

The projective delta functions combine naturally to form the supersymmetric delta functions. For example, consider the 'wrong sign' $\tilde{\delta}$-function

$$
\begin{aligned}
\tilde{\delta}_{\mathcal{N}-4-n}^{(3 \mid \mathcal{N})}(\mathrm{W}, \mathrm{Y}) & =\int_{-\infty}^{\infty} \frac{\mathrm{d} t}{t^{1+n}} \delta^{(4 \mid \mathcal{N})}(\mathrm{W}-t \mathrm{Y}) \\
& :=\int_{-\infty}^{\infty} \frac{\mathrm{d} t}{t^{1+n}} \delta^{(4)}(W-t Y) \prod_{i=1}^{\mathcal{N}}\left(\chi_{i}-t \psi_{i}\right)
\end{aligned}
$$

where $\mathrm{W}=\left(W_{\alpha}, \chi_{i}\right)$ and $\mathrm{Y}=\left(Y_{\beta}, \psi_{j}\right)$. By including a factor of $\operatorname{sgn}(t)$ in the measure, we can also define a supersymmetric $\delta$-function with the correct sign behaviour, but because

\footnotetext{
${ }^{10}$ Just as ordinary homogeneous functions can be thought of as sections of a line bundle $\mathcal{O}(n)$ on the projective space, such 'wrong sign' functions correspond to sections of another family of invariantly defined line bundles $\widetilde{\mathcal{O}}(n)$ on the projective space.
} 
of the twistor structure of the BCFW recursion relations, we will find that (3.11) is more directly useful. When $\mathcal{N}=4$ and $n=0$, (3.11) has homogeneity zero in both its arguments (as appropriate for $\mathcal{N}=4$ twistor supermultiplets), whereas for $\mathcal{N}=8 \mathrm{SG}$ we will most frequently set $n=1$ so that $\tilde{\delta}^{(3 \mid 8)}(\mathrm{W}, \mathrm{Y})$ has homogeneities $(3,1)$ in $(\mathrm{W}, \mathrm{Y})$. In each of these cases, we omit the subscript.

One can also define a family of descendant $\delta$-functions and $\tilde{\delta}$-functions that enforce collinearity and coplanarity etc. of more twistors, rather than just coincidence. In particular, we will make use of the $\mathcal{N}=4$ and $\mathcal{N}=8$ collinear $\tilde{\delta}$-functions

$$
\begin{aligned}
& \tilde{\delta}^{(2 \mid 4)}\left(\mathrm{W}_{1} ; \mathrm{W}_{2}, \mathrm{~W}_{3}\right):=\int_{\mathbb{R}^{2}} \frac{\mathrm{d} s}{s} \frac{\mathrm{d} t}{t} \delta^{(4 \mid 4)}\left(\mathrm{W}_{1}-s \mathrm{~W}_{2}-t \mathrm{~W}_{3}\right) \\
& \tilde{\delta}^{(2 \mid 8)}\left(\mathrm{W}_{1} ; \mathrm{W}_{2}, \mathrm{~W}_{3}\right):=\int_{\mathbb{R}^{2}} \frac{\mathrm{d} s}{s^{2}} \frac{\mathrm{d} t}{t^{2}} \delta^{(4 \mid 8)}\left(\mathrm{W}_{1}-s \mathrm{~W}_{2}-t \mathrm{~W}_{3}\right) .
\end{aligned}
$$

which are again superconformally invariant by construction. These collinear $\tilde{\delta}$-functions have correct sign behaviour for $\mathrm{W}_{1}$, but wrong sign behaviour for $\mathrm{W}_{2}$ and $\mathrm{W}_{3}$. The completely right sign untilded collinear $\delta$-function is (for $\mathcal{N}=4$ )

$$
\begin{aligned}
\delta^{(2 \mid 4)}\left(\mathrm{W}_{1}, \mathrm{~W}_{2}, \mathrm{~W}_{3}\right) & :=\int_{\mathbb{R}^{2}} \frac{\mathrm{d} s}{|s|} \frac{\mathrm{d} t}{|t|} \delta^{(4 \mid 4)}\left(\mathrm{W}_{1}-s \mathrm{~W}_{2}-t \mathrm{~W}_{3}\right) \\
& =\operatorname{sgn}\left(\left\langle\mathrm{W}_{1} \mathrm{~W}_{2}\right\rangle\left\langle\mathrm{W}_{3} \mathrm{~W}_{1}\right\rangle\right) \tilde{\delta}^{(2 \mid 4)}\left(\mathrm{W}_{1} ; \mathrm{W}_{2}, \mathrm{~W}_{3}\right),
\end{aligned}
$$

where the second line follows from using the delta functions in the $\lambda_{A}$ co-ordinates to evaluate $s=\langle 13\rangle /\langle 23\rangle$ and $t=\langle 12\rangle /\langle 32\rangle$ so that

$$
\operatorname{sgn}(s t)=\operatorname{sgn}\left(\left\langle\mathrm{W}_{1} \mathrm{~W}_{2}\right\rangle\left\langle\mathrm{W}_{3} \mathrm{~W}_{1}\right\rangle\right) .
$$

For $\mathcal{N}=4,(3.13)$ is invariant under scaling of each of its arguments, and performing elementary substitutions shows that in fact it is totally symmetric under exchange.

Non-projectively $\mathrm{W}_{1}-s \mathrm{~W}_{2}-t \mathrm{~W}_{3}$ can only vanish when $\mathrm{W}_{1}$ lies in the two-plane containing the origin that is spanned by $\mathrm{W}_{2}$ and $\mathrm{W}_{3}$. Therefore $\tilde{\delta}^{(2 \mid \mathcal{N})}\left(\mathrm{W}_{1} ; \mathrm{W}_{2}, \mathrm{~W}_{3}\right)$ and $\delta^{(2 \mid \mathcal{N})}\left(\mathrm{W}_{1}, \mathrm{~W}_{2}, \mathrm{~W}_{3}\right)$ have co-dimension $2 \mid \mathcal{N}$ support on the set where $\mathrm{W}_{1}, \mathrm{~W}_{2}$ and $\mathrm{W}_{3}$ are collinear. Again, in order to explicitly perform the $s, t$ integrals we must break conformal invariance: The invariant condition for collinearity (in the bosonic twistor space) is

$$
\varepsilon^{\alpha \beta \gamma \delta} W_{1 \beta} W_{2 \gamma} W_{3 \delta}=0
$$

but there is no conformally invariant or global way to take just two of these four equations. ${ }^{11}$

A natural extension to the coplanarity $\tilde{\delta}$-function is

$$
\tilde{\delta}^{(1 \mid \mathcal{N})}\left(\mathrm{W}_{1}, \mathrm{~W}_{2}, \mathrm{~W}_{3}, \mathrm{~W}_{4}\right):=\int_{\mathbb{R}^{3}} \frac{\mathrm{d} r}{r} \frac{\mathrm{d} s}{s} \frac{\mathrm{d} t}{t} \delta^{(4 \mid \mathcal{N})}\left(\mathrm{W}_{1}-r \mathrm{~W}_{2}-s \mathrm{~W}_{3}-t \mathrm{~W}_{4}\right) .
$$

and similarly for $\delta^{(1 \mid \mathcal{N})}$. Differently weighted versions are obtained by including various powers of the $r, s, t, \ldots$ variables in the measures, together with signs to change the right/wrong sign behaviours. (For example, replacing $\mathrm{d} r / r \rightarrow \mathrm{d} r /|r|$ produces a version that is right sign in $\mathrm{W}_{1}$ and $\mathrm{W}_{2}$ and wrong sign in $\mathrm{W}_{3}$ and $\mathrm{W}_{4}$ )

\footnotetext{
${ }^{11}$ There is a Poincaré invariant choice in this case, which leads to the explicit form (3.2) of the three-point MHV amplitude.
} 


\subsubsection{The Hilbert transform and the sgn function}

The original Hilbert transform is a complex structure on functions on the real line that fall off as $O(1 / x)$ as $x \rightarrow \infty$. It is given by the formula

$$
H[f](x)=\text { p.v. } \frac{1}{\pi} \int_{\mathbb{R}} \frac{\mathrm{d} y}{y} f(x-y), \quad H[H[f]]=-f .
$$

One way to view the Hilbert transform is to say that if $f=\operatorname{Re}(F)$ where $F$ is a complex function that is holomorphic on the upper half plane, then $H[f]=\operatorname{Im}(F)$. This follows by expressing the principal value regularisation for the distribution $1 / y$ as

$$
\frac{1}{y}=\frac{1}{2} \lim _{\epsilon \rightarrow 0}\left(\frac{1}{y+i \epsilon}+\frac{1}{y-i \epsilon}\right)
$$

the right hand side give rise to the Cauchy integral formula for i $F$ and its complex conjugate in terms of an integral of $f$ along the real axis.

We can extend the idea of the Hilbert transform to distributions on twistor space (or more general projective spaces) as follows: choose a line in twistor space by fixing a point $A$ and then perform a Hilbert transform along the line joining $W$ to $A$. That is, we make the following

Definition 1 The Hilbert transform of a function (or distribution) $f(W)$ in the direction $A$ is

$$
\mathrm{H}_{A}[f](W):=\frac{1}{\pi} \int_{-\infty}^{\infty} \frac{\mathrm{d} t}{t} f\left(W_{\alpha}-t A_{\alpha}\right),
$$

where the integral is understood by the principal value prescription.

Thus, the basic wrong sign $\tilde{\delta}$-functions may be viewed as Hilbert transforms of the nonprojective $\delta$-function, for example

$$
\begin{aligned}
\tilde{\delta}^{(3 \mid \mathcal{N})}\left(\mathrm{W}_{1}, \mathrm{~W}_{2}\right) & =\pi \mathrm{H}_{\mathrm{W}_{2}}\left[\delta^{(4 \mid \mathcal{N})}\left(\mathrm{W}_{1}\right)\right] \\
\tilde{\delta}^{(2 \mid \mathcal{N})}\left(\mathrm{W}_{1} ; \mathrm{W}_{2}, \mathrm{~W}_{3}\right) & =\pi^{2} \mathrm{H}_{\mathrm{W}_{2}}\left[\mathrm{H}_{\mathrm{W}_{3}}\left[\delta^{(4 \mid \mathcal{N})}\left(\mathrm{W}_{1}\right)\right]\right]
\end{aligned}
$$

and so on.

The Hilbert transform has a useful interplay with the Fourier transform. Representing a (non-projective) distribution $f(W)$ by its Fourier transform $F(Z)$ we can write

$$
\begin{aligned}
\int_{-\infty}^{\infty} \frac{\mathrm{d} t}{t} f\left(W_{\alpha}-t A_{\alpha}\right) & =\int \frac{\mathrm{d} t}{t} \mathrm{~d}^{4} Z \mathrm{e}^{\mathrm{i}(W-t A) \cdot Z} F(Z) \\
& =-\mathrm{i} \pi \int \mathrm{d}^{4} Z \operatorname{sgn}(A \cdot Z) \mathrm{e}^{\mathrm{i} W \cdot Z} F(Z) \\
& =\mathrm{i} \pi \operatorname{sgn}\left(\mathrm{i} A \cdot \frac{\partial}{\partial W}\right) f(W)
\end{aligned}
$$

where in the second line we use the fact that

$$
\text { p.v. } \int_{-\infty}^{\infty} \frac{\mathrm{d} t}{t} \mathrm{e}^{-\mathrm{i} a t}=-\mathrm{i} \pi \operatorname{sgn} a,
$$


and in the last line follows by setting ${ }^{12} Z^{\alpha}=-\mathrm{i} \partial / \partial W_{\alpha}$. At least formally, this allows us to express the weighted projective $\delta$-functions in terms of pseudo-differential operators. In particular, when $\mathcal{N}=4$ we have $\mathrm{e}^{13}$

$$
\begin{aligned}
\tilde{\delta}^{(3 \mid 4)}\left(\mathrm{W}_{1}, \mathrm{~W}_{2}\right) & =\int \frac{\mathrm{d} t}{t} \delta^{(4 \mid 4)}\left(\mathrm{W}_{1}-t \mathrm{~W}_{2}\right) \\
& =\mathrm{i} \pi \operatorname{sgn}\left(\mathrm{iW}_{2} \cdot \frac{\partial}{\partial \mathrm{W}}\right) \delta^{(4 \mid 4)}\left(\mathrm{W}_{1}\right)
\end{aligned}
$$

and

$$
\begin{aligned}
\tilde{\delta}^{(2 \mid 4)}\left(\mathrm{W}_{1} ; \mathrm{W}_{2}, \mathrm{~W}_{3}\right) & =\int \frac{\mathrm{d} s}{s} \frac{\mathrm{d} t}{t} \delta^{(4 \mid 4)}\left(\mathrm{W}_{1}-s \mathrm{~W}_{2}-t \mathrm{~W}_{3}\right) \\
& =(\mathrm{i} \pi)^{2} \operatorname{sgn}\left(\mathrm{iW}_{2} \cdot \frac{\partial}{\partial \mathrm{W}_{1}} \mathrm{iW}_{3} \cdot \frac{\partial}{\partial \mathrm{W}_{1}}\right) \delta^{(4 \mid 4)}\left(\mathrm{W}_{1}\right),
\end{aligned}
$$

whereas when $\mathcal{N}=8$ the principal value integral (see e.g. [69])

$$
\text { p.v. } \int_{-\infty}^{\infty} \frac{\mathrm{d} t}{t^{2}} \mathrm{e}^{-\mathrm{i} a t}=-\pi|a|
$$

gives

$$
\begin{aligned}
\tilde{\delta}^{(3 \mid 8)}\left(\mathrm{W}_{1}, \mathrm{~W}_{2}\right) & =\int \frac{\mathrm{d} t}{t^{2}} \delta^{(4 \mid 8)}\left(\mathrm{W}_{1}-t \mathrm{~W}_{2}\right) \\
& =-\pi\left|\mathrm{i} \mathrm{W}_{2} \cdot \frac{\partial}{\partial \mathrm{W}_{1}}\right| \delta^{(4 \mid 8)}\left(\mathrm{W}_{1}\right)
\end{aligned}
$$

and

$$
\begin{aligned}
\tilde{\delta}^{(2 \mid 8)}\left(\mathrm{W}_{1} ; \mathrm{W}_{2}, \mathrm{~W}_{3}\right) & =\int \frac{\mathrm{d} s}{s^{2}} \frac{\mathrm{d} t}{t^{2}} \delta^{(4 \mid 8)}\left(\mathrm{W}_{1}-s \mathrm{~W}_{2}-t \mathrm{~W}_{3}\right) \\
& =\pi^{2}\left|\mathrm{iW}_{2} \cdot \frac{\partial}{\partial \mathrm{W}_{1}} \mathrm{iW}_{3} \cdot \frac{\partial}{\partial \mathrm{W}_{1}}\right| \delta^{(4 \mid 8)}\left(\mathrm{W}_{1}\right)
\end{aligned}
$$

This notation helpfully encodes the scaling behaviour, and it will often be convenient to write both the basic seed amplitudes and the recursion relations themselves in terms of these sign operators.

\subsection{Seed amplitudes in $\mathcal{N}=4 \mathrm{SYM}$}

We now have all the necessary ingredients to state the twistor space form of the basic three-point amplitudes in a way that clarifies their conformal properties. In this section,

\footnotetext{
${ }^{12}$ Note that $\mathrm{i} A \cdot \frac{\partial}{\partial W}$ is Hermitian.

${ }^{13}$ We can similarly write formally

$$
\log \left(\mathrm{i} A \cdot \frac{\partial}{\partial W}\right) f(W)=\int \frac{\mathrm{d} t}{|t|} f(W+t A)
$$

although this integral needs to be regularised more carefully [69]. So similarly,

$$
\delta^{(3 \mid 4)}\left(W_{1}, W_{2}\right)=\log \left(\mathrm{i} W_{2} \cdot \frac{\partial}{\partial W_{1}}\right) \delta^{(4 \mid 4)}\left(W_{1}\right) .
$$
}

We will not have so much use for this formula however. 
we confine ourselves to a discussion of $\mathcal{N}=4 \mathrm{SYM}$, postponing the (largely parallel) case of $\mathcal{N}=8 \mathrm{SG}$ until section 6 .

The twistor form of the 3-particle MHV super-amplitude in $\mathcal{N}=4 \mathrm{SYM}$ may be written as

$$
\begin{aligned}
\mathcal{A}_{\mathrm{MHV}}\left(\mathrm{W}_{1}, \mathrm{~W}_{2}, \mathrm{~W}_{3}\right) & =\operatorname{sgn}\left(\left\langle\mathrm{W}_{2} \mathrm{~W}_{3}\right\rangle\right) \tilde{\delta}^{(2 \mid 4)}\left(\mathrm{W}_{1} ; \mathrm{W}_{2}, \mathrm{~W}_{3}\right) \\
& =\operatorname{sgn}\left(\left\langle\mathrm{W}_{1} \mathrm{~W}_{2}\right\rangle\left\langle\mathrm{W}_{2} \mathrm{~W}_{3}\right\rangle\left\langle\mathrm{W}_{3} \mathrm{~W}_{1}\right\rangle\right) \delta^{(2 \mid 4)}\left(\mathrm{W}_{1}, \mathrm{~W}_{2}, \mathrm{~W}_{3}\right) .
\end{aligned}
$$

where $\left\langle\mathrm{W}_{i} \mathrm{~W}_{j}\right\rangle=\left\langle\lambda_{i} \lambda_{j}\right\rangle$ is the usual spinor product of the $\lambda$-parts of the spinor. Thus the complete super-amplitude is a superconformally invariant $\delta$-function imposing collinearity of $\mathrm{W}_{1}, \mathrm{~W}_{2}, \mathrm{~W}_{3}$, times a sign factor. The sign factor ensures that (3.28) is antisymmetric under the exchange of any two points, compensating the antisymmetry of the colour factor $\operatorname{Tr}\left(T_{1}\left[T_{2}, T_{3}\right]\right)$. Since the sign only depends on the ordering of the three twistors, we see that the twistor amplitude is completely geometric: it depends on the three ordered points on a line.

Remarkably, the extension of this amplitude to the $n$-point MHV amplitude is the product

$$
\mathcal{A}_{\mathrm{MHV}}\left(\mathrm{W}_{1}, \ldots, \mathrm{W}_{n}\right)=(-1)^{n-3} \prod_{i=3}^{n} \mathcal{A}_{\mathrm{MHV}}\left(\mathrm{W}_{1}, \mathrm{~W}_{i-1}, \mathrm{~W}_{i}\right),
$$

as shown by half Fourier transform in appendix $\mathrm{C}$ and obtained from twistor BCFW recursion below. Each three-particle MHV amplitude enforces collinearity of $\mathrm{W}_{1}, \mathrm{~W}_{i-1}$ and $\mathrm{W}_{i}$, so the product of such three-particle amplitudes has the well-known collinear support in twistor space. Again, the amplitude is purely geometric; there are no extra spinor or twistor products. The cyclic symmetry of the MHV amplitude is not explicit, but follows from (3.29) and the cyclic symmetry of the 4-point amplitude

$$
\mathcal{A}_{\mathrm{MHV}}(1,2,3) \mathcal{A}_{\mathrm{MHV}}(1,3,4)=\mathcal{A}_{\mathrm{MHV}}(2,3,4) \mathcal{A}_{\mathrm{MHV}}(2,4,1) .
$$

This four-point identity is easily proved using the three-point amplitude in the form (3.2).

The three-point $\overline{\mathrm{MHV}}$ amplitude, given in on-shell momentum superspace by [34]

$$
A_{\overline{\mathrm{MHV}}}\left(p_{1}, p_{2}, p_{3}\right)=\frac{\delta^{(4)}\left(p_{1}+p_{2}+p_{3}\right) \delta^{(4)}\left(\eta_{1}[23]+\eta_{2}[31]+\eta_{3}[12]\right)}{[12][23][31]},
$$

has the twistor space representation

$$
\mathcal{A}_{\overline{\mathrm{MHV}}}\left(\mathrm{W}_{1}, \mathrm{~W}_{2}, \mathrm{~W}_{3}\right)=\operatorname{sgn}\left(\left[\frac{\partial}{\partial \mathrm{W}_{2}} \frac{\partial}{\partial \mathrm{W}_{3}}\right]\right) \tilde{\delta}^{(3 \mid 4)}\left(\mathrm{W}_{1}, \mathrm{~W}_{2}\right) \tilde{\delta}^{(3 \mid 4)}\left(\mathrm{W}_{1}, \mathrm{~W}_{3}\right) .
$$

The operator $\left.\operatorname{sgn}\left[\partial_{2} \partial_{3}\right]\right)$ is a pseudo-differential operator that is closely related to the Hilbert transform. Like the Hilbert transform, it can be easily understood in terms of its Fourier transform (whence it arose). $\mathcal{A}_{\overline{\mathrm{MHV}}}$ may also be written explicitly as

$$
\begin{aligned}
& \mathcal{A}_{\overline{\mathrm{MHV}}}\left(\mathrm{W}_{1}, \mathrm{~W}_{2}, \mathrm{~W}_{3}\right)=\frac{\lambda_{1}}{\lambda_{2}} \frac{\lambda_{1}}{\lambda_{3}} \delta(\langle 12\rangle) \delta(\langle 13\rangle) \times \\
& \quad \times \delta^{\prime}\left(\left(\mu_{2}^{A^{\prime}}-\frac{\lambda_{2}}{\lambda_{1}} \mu_{1}^{A^{\prime}}\right)\left(\mu_{3 A^{\prime}}-\frac{\lambda_{3}}{\lambda_{1}} \mu_{1 A^{\prime}}\right)\right) \delta^{(0 \mid 4)}\left(\chi_{2}-\frac{\lambda_{2}}{\lambda_{1}} \chi_{1}\right) \delta^{(0 \mid 4)}\left(\chi_{3}-\frac{\lambda_{3}}{\lambda_{1}} \chi_{1}\right)
\end{aligned}
$$


which follows directly from the half Fourier transform. Again, this explicit representation obscures the conformal properties and in practice the implicit form (3.32) will actually be more useful. It is easy to show that (3.32) is antisymmetric under exchange of any two external states, again compensating the antisymmetry of the colour factor.

Using the sign-function representations of the delta functions, we can also write the three-point amplitudes as

$$
\begin{aligned}
& \mathcal{A}_{\mathrm{MHV}}\left(\mathrm{W}_{1}, \mathrm{~W}_{2}, \mathrm{~W}_{3}\right)=(\mathrm{i} \pi)^{2} \operatorname{sgn}\left(\langle 23\rangle \mathrm{iW}_{2} \cdot \partial_{\mathrm{W}_{1}} \mathrm{iW}_{3} \cdot \partial_{\mathrm{W}_{1}}\right) \delta^{(4 \mid 4)}\left(\mathrm{W}_{1}\right) \\
& \mathcal{A}_{\overline{\mathrm{MHV}}}\left(\mathrm{W}_{1}, \mathrm{~W}_{2}, \mathrm{~W}_{3}\right)=(\mathrm{i} \pi)^{2} \operatorname{sgn}\left(\left[\partial_{\mathrm{W}_{2}} \partial_{\mathrm{W}_{3}}\right] \mathrm{iW}_{1} \cdot \partial_{\mathrm{W}_{2}} \mathrm{iW}_{1} \cdot \partial_{\mathrm{W}_{3}}\right) \delta^{(4 \mid 4)}\left(\mathrm{W}_{2}\right) \delta^{(4 \mid 4)}\left(\mathrm{W}_{3}\right) .
\end{aligned}
$$

This representation may seem rather formal, but it is well-defined as a distribution and in any case is completely equivalent to the concrete forms (3.2) \& (3.33). Alternatively, one can obtain an ambidextrous representation of the amplitudes by writing the $\delta^{(4 \mid 4)}$-functions in terms of Fourier transforms, as is done in [46] and as we discuss further in section 7. The differential operators inside the sign functions in (3.34) play a prominent role in what follows. For future reference, we therefore define

$$
\mathcal{H}_{j k}^{i}:=(\mathrm{i} \pi)^{2} \operatorname{sgn}\left(\left\langle\mathrm{W}_{j} \mathrm{~W}_{k}\right\rangle \mathrm{iW}_{j} \cdot \frac{\partial}{\partial \mathrm{W}_{i}} \mathrm{iW}_{k} \cdot \frac{\partial}{\partial \mathrm{W}_{i}}\right)
$$

and similarly

$$
\widetilde{\mathcal{H}}_{i}^{j k}:=(\mathrm{i} \pi)^{2} \operatorname{sgn}\left(\left[\frac{\partial}{\partial \mathrm{W}_{j}} \frac{\partial}{\partial \mathrm{W}_{k}}\right] \mathrm{iW}_{i} \cdot \frac{\partial}{\partial \mathrm{W}_{j}} \mathrm{iW}_{i} \cdot \frac{\partial}{\partial \mathrm{W}_{k}}\right) .
$$

These $\mathcal{H}_{j k}^{i}$ and $\widetilde{\mathcal{H}}_{k}^{i j}$ operators are conjugate, in the sense that they are related by making the replacements $\mathrm{W}_{i} \leftrightarrow \partial / \partial \mathrm{W}_{i}$ and exchanging the infinity twistor and its dual $I^{\alpha \beta} \leftrightarrow I_{\alpha \beta}$. Each of these operators will play an important role in the construction of general tree amplitudes. In this representation, the cyclic symmetry of the three-point amplitude is the identity

$$
\mathcal{H}_{23}^{1} \delta^{(4 \mid 4)}\left(\mathrm{W}_{1}\right)=\mathcal{H}_{31}^{2} \delta^{(4 \mid 4)}\left(\mathrm{W}_{2}\right),
$$

while the cyclic symmetry (3.30) of the four-point amplitude is

$$
\mathcal{H}_{13}^{2} \mathcal{H}_{13}^{4} \delta^{(4 \mid 4)}\left(\mathrm{W}_{2}\right) \delta^{(4 \mid 4)}\left(\mathrm{W}_{4}\right)=\mathcal{H}_{24}^{3} \mathcal{H}_{24}^{1} \delta^{(4 \mid 4)}\left(\mathrm{W}_{3}\right) \delta^{(4 \mid 4)}\left(\mathrm{W}_{1}\right)
$$

\subsection{On conformal invariance}

The results of the previous subsection showed that seed amplitudes in $\mathcal{N}=4 \mathrm{SYM}$ are one or two superconformally invariant $\delta$-functions, dressed by certain signs (which may not be locally defined). Although the delta functions in (3.28) \& (3.32) are manifestly superconformally invariant, the factors of $\operatorname{sgn}\left(\left\langle\mathrm{W}_{i} \mathrm{~W}_{j}\right\rangle\right)$ and the operator $\operatorname{sgn}\left(\left[\partial_{2} \partial_{3}\right]\right)$ are not. No choice of tilded or untilded $\delta$-function removes all of these signs, which are necessary for the amplitudes to have the correct cyclic and exchange properties.

Is this a failure of conformal invariance, or merely a failure to make invariance manifest? Consider the three-point MHV amplitude, written in the second form of (3.28) which makes the exchange properties transparent. On the support of the delta function, the three twistors are collinear and the sign factor just depends on the ordering of the points along 
this line. Thus, if we could consistently orientate all the lines in twistor space, we would be able to replace the factor $\operatorname{sgn}\left(\left\langle\mathrm{W}_{1} \mathrm{~W}_{2}\right\rangle\left\langle\mathrm{W}_{2} \mathrm{~W}_{3}\right\rangle\left\langle\mathrm{W}_{3} \mathrm{~W}_{1}\right\rangle\right)$ by the prescription that the collinear delta-function is to be multiplied by +1 if the ordering of the three twistors agrees with the chosen orientation, and by -1 if not. The amplitude would then be conformally invariant. However, there is a topological obstruction to doing this: an oriented line in $\mathbb{R P}^{3}$ can always be continuously deformed so that it comes back to itself with the opposite orientation (e.g. by rotating it through $\pi$ about an axis that is perpendicular to the line, thinking of it in affine $\mathbb{R}^{3}$ ). Globally, the space of $\mathbb{R} \mathbb{P}^{1} \mathrm{~s}$ inside $\mathbb{R} \mathbb{P}^{3}$ is conformally compactified split signature space-time with topology $\left(S^{2} \times S^{2}\right) / \mathbb{Z}_{2}$. This space has fundamental group $\mathbb{Z}_{2}$, and this fundamental group precisely corresponds to the possible orientations of the twistor line. ${ }^{14}$

After removing a line $I$ 'at infinity', one can orientate all the $\mathbb{R} \mathbb{P}^{1} \mathrm{~s}$ that do not meet this line. The remaining twistor space fibres over $\mathbb{R P}^{1}$ and we can fix the orientation on any line that does not meet $I$ by pulling back the orientation of this $\mathbb{R P}^{1}$. Equivalently, removing a line $I$ from twistor space removes a point $i$ from conformally compactified space-time. The space of twistor lines that do not intersect $I$ corresponds to the region of conformally compactified space-time that is not null separated from the point $i$, in other words affine space-time $\mathbb{R}^{2,2}$. Thus, provided one stays within a single copy of split signature affine space-time, the sign factors in (3.28) just amount to an overall sign that may be consistently chosen. However, there is no consistent way to extend this over the whole of $\mathbb{R} \mathbb{P}^{3}$, and conformal invariance is genuinely broken.

How do we reconcile this with the fact that momentum space amplitudes are annihilated by all the superconformal generators (see e.g. [1])? Let us examine in detail how conformal invariance is broken. Acting on the three-point MHV amplitude (in the form of the second equation of (3.28)) with the conformal generators $J_{\alpha}{ }^{\beta}=\sum_{i=1}^{3} \mathrm{~W}_{i \alpha} \partial / \partial \mathrm{W}_{i \beta}$, the only possible contribution comes from the sign function. We obtain

$$
J_{\alpha}^{\beta} \mathcal{A}_{\mathrm{MHV}}\left(\mathrm{W}_{1}, \mathrm{~W}_{2}, \mathrm{~W}_{3}\right)=\left(\mathrm{W}_{2 \alpha} I^{\beta \gamma} \mathrm{W}_{3 \gamma}-\mathrm{W}_{3 \alpha} I^{\beta \gamma} \mathrm{W}_{2 \gamma}\right) \delta\left(\left\langle\mathrm{W}_{2} \mathrm{~W}_{3}\right\rangle\right) \tilde{\delta}^{(2 \mid 4)}\left(\mathrm{W}_{1} ; \mathrm{W}_{2}, \mathrm{~W}_{3}\right) .
$$

On the support of the delta functions in this expression, the $\mathrm{W}_{i}$ are all collinear and $\left\langle\mathrm{W}_{2} \mathrm{~W}_{3}\right\rangle=0$. Geometrically, the condition $\left\langle\mathrm{W}_{2} \mathrm{~W}_{3}\right\rangle=0$ means that $\mathrm{W}_{2}$ and $\mathrm{W}_{3}$ lie in the same plane through the line 'at infinity' $\left(I^{\alpha \beta} \mathrm{W}_{\alpha}=0\right.$, or $\left.\lambda_{A}=0\right)$. So the delta functions in (3.39) give support only when the $\mathrm{W}_{i}$ all lie on a line that intersects the line at infinity. On such lines, the coefficient $\mathrm{W}_{2 \alpha} I^{\beta \gamma} \mathrm{W}_{3 \gamma}-\mathrm{W}_{3 \alpha} I^{\beta \gamma} \mathrm{W}_{2 \gamma}$ does not vanish (unless $\mathrm{W}_{2}$ and $\mathrm{W}_{3}$ actually coincide). Thus, the failure of conformal invariance occurs where all three particles' $\lambda_{A}$ spinors are proportional. This is the most singular part of the momentum space amplitude - a momentum space calculation (or one based on the explicit twistor form (3.2)) could only uncover the failure of conformal invariance with a careful analysis of anomalous terms in the action of the conformal generators in the triple-collinear limit.

\footnotetext{
${ }^{14} \mathrm{~A}$ related fact is that the integral in the X-ray transform requires an orientation, and so gives rise to massless fields that have wrong-sign behaviour with respect to this $\mathbb{Z}_{2}$, i.e., they are wrong-sign sections of the line bundle of functions of homogeneity -1 on the 4 -quadric with signature $(3,3)$ in $\mathbb{R} \mathbb{P}^{5}$. This 'wrong-sign' behaviour is not correlated with that of the amplitude.
} 
However, although the failure of conformal invariance is associated with the collinear singularities of the momentum space amplitudes, note that nothing singular is happening in twistor space. Given a line in 'affine' twistor space, a collinear singularity occurs when two or more marked points on this line collide - this process is conformally invariant. By contrast, the violation of conformal invariance above is associated with support on lines that intersect $I$. No twistors need collide, and from the point of view of the conformally compactified space, this line is on the same footing as any other. Moreover, the collinear delta function $\delta^{(2 \mid 4)}\left(\mathrm{W}_{1}, \mathrm{~W}_{2}, \mathrm{~W}_{3}\right)$ corresponds to

$$
\frac{\delta^{(4 \mid 4)}\left(\sum_{i=1}^{3}|i\rangle \llbracket i \|\right)}{|\langle 12\rangle\langle 23\rangle\langle 31\rangle|}
$$

on momentum space, so (at least away from singularities) it equals ${ }^{15} \pm A_{\mathrm{MHV}}(1,2,3)$. Thus, on the open region of momentum space with collinear singularities removed, (3.40) is likewise annihilated by all the generators of the superconformal algebra. However, under a finite conformal transformation, (3.40) fits together across the singularities in a way which is conformally invariant, while the amplitude itself does not.

The conformal properties of the three-point $\overline{\mathrm{MHV}}$ amplitude follows similarly from a CPT transformation (or the Fourier transform of section 7). Although the $n$-particle MHV amplitudes might at first sight appear worse, many of the sign factors cancel: Arrange the 3-point factors in (3.29) pair-wise, and use cyclic symmetry and the first line of (3.28) to ensure that the only sign factors are $\operatorname{sgn}\langle 1 i\rangle$, occurring in both the $i^{\text {th }}$ and $(i+1)^{\text {th }}$ term. These signs then cancel. With this cancellation, the even-point MHV amplitudes are manifestly conformally invariant. The odd-point amplitudes still end up with the one threepoint sub-amplitude in the product (3.29) whose conformal breaking sign factor cannot be made to cancel. This is consistent with the topological argument because the relation

$$
\mathcal{A}(1,2, \ldots, n-1, n)=(-1)^{n} \mathcal{A}(n, n-1, \ldots, 2,1),
$$

requires that odd-point amplitudes, but not even-point amplitudes, change sign under a reversal of the orientation of points along the line.

\section{BCFW recursion in twistor space}

We now use the half Fourier transform to translate the supersymmetric BCFW recursion relation into twistor space. See [5] for a proof of the original BCFW rule in Yang-Mills, [47] for gravity and $[34,48]$ for the supersymmetric extension.

In both $\mathcal{N}=8 \mathrm{SG}$ and $\mathcal{N}=4 \mathrm{SYM}$, the (super-)BCFW recursion rule states that

$$
\widetilde{A}(1, \ldots, n)=\sum \int \mathrm{d}^{\mathcal{N}} \eta \widetilde{A}_{L}(\hat{1}, 2, \ldots, i,\{-\hat{p}, \eta\}) \frac{1}{p^{2}} \widetilde{A}_{R}(\{\hat{p}, \eta\}, i+1, \ldots, n-1, \hat{n})
$$

where $\widetilde{A}$ denotes a tree-level super-amplitude that has been stripped of its overall momentum conserving $\delta$-function (and, in Yang-Mills, also of its colour factor). The sum is

\footnotetext{
${ }^{15}$ Recall that in split signature, the momentum space spinors are real.
} 
taken over all possible ways of splitting the external states among the two sub-amplitudes, subject to the requirement that the shifted momenta reside in separate sub-amplitudes (and subject to cyclic symmetry in SYM). The integral over the Grassmann variables $\eta$ of the internal supermultiplet accounts for the possible helicity states of the internal particle. The propagator momentum $p$ is defined as usual, i.e.

$$
p:=\sum_{j \in L} p_{j}
$$

where $L$ is the set of external particles attached to the left sub-amplitude. The supermomenta in the sub-amplitudes are shifted compared to the external momenta according to the general prescription (2.2). Similarly, in the sub-amplitudes $\widetilde{A}_{L, R}$, the propagator momentum $p$ is shifted as $p \rightarrow \hat{p}:=p-t|1\rangle[n \mid$. For a given term in the sum in (4.1) (i.e., a given decomposition into sub-amplitudes) the shift parameter $t$ is fixed to the value $t_{*}$ that ensures $\hat{p}^{2}\left(t_{*}\right)=0$. Consequently, all the momenta in $\widetilde{A}_{L, R}$ are null, so these are fully on-shell sub-amplitudes. Note that the $t_{*}$ are real in $(++--)$ signature space-time.

As a preliminary step towards transforming the BCFW relation to twistor space, first restore the momentum-conserving $\delta$-functions to (4.1). One finds

$$
A(1, \ldots, n)=\sum \int \mathrm{d}^{4} p \mathrm{~d}^{\mathcal{N}} \eta \delta^{(4)}\left(-p+\sum_{j \in L} p_{j}\right) \widetilde{A}_{L}\left(t_{*}\right) \frac{1}{p^{2}} \delta^{(4)}\left(p+\sum_{k \in R} p_{k}\right) \widetilde{A}_{R}\left(t_{*}\right)
$$

where now $p$ is a priori unconstrained (and in particular is generically off-shell). We can always obtain a null momentum by projecting the arbitrary momentum $p$ along some fixed null momentum direction, so we can always set

$$
p=\ell-t|1\rangle[n \mid
$$

where $\ell=|\lambda\rangle[\tilde{\lambda} \mid$ is a null but otherwise arbitrary momentum, and $t$ is an arbitrary parameter.

In terms of the $(\ell, t)$ variables, the integration measure and propagator combine to become

$$
\frac{\mathrm{d}^{4} p}{p^{2}}=\operatorname{sgn}(\langle 1|\ell| n]) \frac{\mathrm{d} t}{t} \mathrm{~d}^{3} \ell=\operatorname{sgn}(\langle 1 \lambda\rangle[\tilde{\lambda} n]) \frac{\mathrm{d} t}{t}\left(\langle\lambda \mathrm{d} \lambda\rangle \mathrm{d}^{2} \tilde{\lambda}-[\tilde{\lambda} \mathrm{d} \tilde{\lambda}] \mathrm{d}^{2} \lambda\right)
$$

as in [50]. The sign factor $\operatorname{sgn}(\langle 1|\ell| n])$ arises because the orientation on the $\mathrm{d} t$ factor changes sign with $\langle 1|\ell| n]$. This can be seen from the fact that the momentum light-cone is naturally oriented by the orientation of momentum space, together with the choice of outward normal going from $p^{2}<0$ to $p^{2}>0$. The direction $\mathrm{d} t$ is essentially that of $|1\rangle[n \mid$, and is aligned or anti-aligned with this outgoing normal according to the sign of $\langle 1 \lambda\rangle[\tilde{\lambda} n]$. Therefore we must incorporate this sign in order to have agreement with the given orientation on momentum space. The measure

$$
\mathrm{d}^{3} \ell=\langle\lambda \mathrm{d} \lambda\rangle \mathrm{d}^{2} \tilde{\lambda}-[\tilde{\lambda} \mathrm{d} \tilde{\lambda}] \mathrm{d}^{2} \lambda
$$


on the null cone in momentum space is invariant under the scaling $(\lambda, \tilde{\lambda}) \rightarrow\left(r^{-1} \lambda, r \tilde{\lambda}\right)$ where $r$ is an arbitrary function of the projective spinors $\left[\lambda_{A}\right]$ and $\left[\tilde{\lambda}_{A^{\prime}}\right]$. We can represent this null cone as a rank two bundle over the $\mathbb{R P}^{1}$ factor, co-ordinatised by the $\left[\lambda_{A}\right]$ on the base and $\tilde{\lambda}_{A^{\prime}}$ up the fibre; doing so amounts to restricting $r$ to be a function of $\left[\lambda_{A}\right]$ alone. The measure then reduces to $\mathrm{d}^{3} \ell=\langle\lambda \mathrm{d} \lambda\rangle \mathrm{d}^{2} \tilde{\lambda}$, which combines with the integral over the internal $\eta$ s to give

$$
\frac{\mathrm{d}^{4} p}{p^{2}} \mathrm{~d}^{\mathcal{N}} \eta=\operatorname{sgn}(\langle 1 \lambda\rangle[\tilde{\lambda} n]) \frac{\mathrm{d} t}{t}\langle\lambda \mathrm{d} \lambda\rangle \mathrm{d}^{2 \mid \mathcal{N}} \tilde{\lambda}
$$

Thus the integral and propagator in the BCFW recursion may together be interpreted as an integral over the on-shell momentum superspace of the internal state, together with an integral over the BCFW shift parameter.

In the $(\lambda, \tilde{\lambda}, t)$ variables, the momentum $\delta$-functions in the integral in (4.3) become

$$
\delta^{(4)}\left(-\lambda \tilde{\lambda}+\sum_{j \in L} \hat{p}_{j}(t)\right) \delta^{(4)}\left(\lambda \tilde{\lambda}+\sum_{k \in R} \hat{p}_{k}(t)\right)
$$

which are the $\delta$-functions associated with the sub-amplitudes $A_{L, R}(t)$ for arbitrary values of the shift parameter $t$. However, on the support of these $\delta$-functions, $t$ is fixed to be precisely $t=t_{*}$ and then $\ell=\hat{p}\left(t_{*}\right)$. Hence the $\delta$-functions allow us to replace $\widetilde{A}_{L, R}\left(t_{i}\right)$ by $A_{L, R}(t)$ inside the integral (4.3). Thus we have a form of the BCFW recursion relation in which the propagator has been absorbed into the measure and all the ingredients are manifestly on-shell:

$$
\begin{aligned}
A(1, \ldots, n)=\sum \int \frac{\mathrm{d} t}{t}\langle\lambda \mathrm{d} \lambda\rangle \mathrm{d}^{2 \mid \mathcal{N}} \tilde{\lambda} & \operatorname{sgn}(\langle 1 \lambda\rangle[\tilde{\lambda} n]) \\
& \times A_{L}(\hat{1}, \ldots,\{-\lambda, \tilde{\lambda}, \eta\}) A_{R}(\{\lambda, \tilde{\lambda}, \eta\}, \ldots, \hat{n}) .
\end{aligned}
$$

This form of the BCFW recursion relation is somewhat similar to a completeness relation: One decomposes the amplitude by inserting a complete set of on-shell states. However, such an interpretation does not account for the shift of the external states 1 and $n$, nor the integral over the shift parameter $t$.

\subsection{The i $\epsilon$-prescription on $\mathbb{R}^{2,2}$}

There is a subtlety ${ }^{16}$ in the definition of the propagator measure in (4.5) \& (4.7) because the $1 / t$ factor means that the $t$-integral is singular. We have seen that for tree amplitudes, the integral is performed by integration against a delta-function, and so the regularisation is not urgent, but it nevertheless should in general be regularised, particularly if one wishes to apply these ideas to loop processes.

In quantum field theory in Minkowski space, stability of the vacuum requires that only positive energy states be allowed to propagate towards the future. This is achieved by using the Feynman propagator $\Delta_{F}(x-y)=\langle 0|T \phi(x) \phi(y)| 0\rangle$ which is time-ordered. Using

\footnotetext{
${ }^{16}$ We thank N. Arkani-Hamed for discussions of this point.
} 
the Fourier transform of the time-ordering step functions

$$
\theta\left(x^{0}-y^{0}\right)=-\frac{1}{2 \pi \mathrm{i}} \int_{-\infty}^{\infty} \mathrm{d} E \frac{\mathrm{e}^{-\mathrm{i} E\left(x^{0}-y^{0}\right)}}{E+\mathrm{i} \epsilon}
$$

one arrives at the $\mathrm{i} \epsilon$-prescription $1 / p^{2} \rightarrow 1 /\left(p^{2}+\mathrm{i} \epsilon\right)$ for the momentum space propagator.

However, in this paper we are tied to split signature space-time, $\mathbb{R}^{2,2}$, which makes no distinction between past and future. The light-cone is connected as are the 'time-like' vectors which are now on the same footing as space-like ones. There is no past or future so it does not make sense to ask that positive energy particles propagate 'forwards' in time and negative energy ones 'backwards'. Correspondingly, in split signature momentum space, the natural choice of $\mathrm{i} \epsilon$ prescription is

$$
\frac{1}{p^{2}} \rightarrow \frac{1}{2}\left(\frac{1}{p^{2}+\mathrm{i} \epsilon}+\frac{1}{p^{2}-\mathrm{i} \epsilon}\right)
$$

We will therefore adopt this prescription when we need to. Thus, the measure in (4.5) can be written as

$$
\begin{aligned}
\frac{\mathrm{d}^{4} p}{p^{2}} & \rightarrow \frac{1}{2}\left(\frac{\mathrm{d}^{4} p}{p^{2}+\mathrm{i} \epsilon}+\frac{\mathrm{d}^{4} p}{p^{2}-\mathrm{i} \epsilon}\right) \\
& =\frac{1}{2}\left(\frac{\mathrm{d} t}{t+\mathrm{i} \epsilon}+\frac{\mathrm{d} t}{t-\mathrm{i} \epsilon}\right) \operatorname{sgn}(\langle 1|\ell| n]) \mathrm{d}^{3} \ell,
\end{aligned}
$$

and this amounts to treating the $\mathrm{d} t$ integral via a Cauchy principal value integral. Although we will often write the abbreviated form (4.5), we will take the $\mathrm{d} t$ integral to be a principal value integral. (This is in contrast to the proof [5] of the BCFW relations which treats it as an $S^{1}$ contour integral.) This wont make any difference to tree level calculations where the integral is determined by delta-functions, but this will make a difference for loops.

\subsection{Transform to twistor space}

We will now take the half Fourier transform of equation (4.9) with respect to the $\tilde{\lambda}$ variables of the external states and substitute in the inverse half Fourier transform from twistor space for the sub-amplitudes. On the lhs, this is just the definition of the twistor super-amplitude:

$$
\mathcal{A}\left(\mathrm{W}_{1}, \ldots, \mathrm{W}_{n}\right):=\int \prod_{i=1}^{n} \mathrm{~d}^{2 \mid \mathcal{N}} \tilde{\lambda}_{i} \mathrm{e}^{\mathrm{i} \llbracket \mu_{i} \tilde{\lambda}_{i} \rrbracket} A(1, \ldots, n) .
$$

Whereas on the right hand side we obtain

$$
\begin{aligned}
& A_{L}(t)=\int \frac{\mathrm{d}^{2 \mid \mathcal{N}} \mu}{(2 \pi)^{2}} \mathrm{e}^{\mathrm{i} \llbracket \mu \tilde{\lambda} \rrbracket} \prod_{j \in L} \frac{\mathrm{d}^{2 \mid \mathcal{N}} \mu_{j}^{\prime}}{(2 \pi)^{2}} \mathrm{e}^{-\mathrm{i} \llbracket \mu_{j}^{\prime} \hat{\tilde{\lambda}}_{j}(t) \rrbracket} \mathcal{A}_{L}\left(\mathrm{~W}_{1}^{\prime}, \ldots, \mathrm{W}\right) \\
& \left.A_{R}(t)=\int \frac{\mathrm{d}^{2 \mid \mathcal{N}} \mu^{\prime}}{(2 \pi)^{2}} \mathrm{e}^{-\mathrm{i} \llbracket \mu^{\prime} \tilde{\lambda} \rrbracket} \prod_{k \in R} \frac{\mathrm{d}^{2 \mid \mathcal{N}} \mu_{k}^{\prime}}{(2 \pi)^{2}} \mathrm{e}^{-\mathrm{i} \llbracket \mu_{k}^{\prime} \tilde{\lambda} \rrbracket} \mathcal{A}_{R}\left(\left\{\lambda, \mu^{\prime}, \chi^{\prime}\right\}, \ldots,\left\{\lambda_{n}-t \lambda_{1}, \mu_{n}^{\prime}, \chi_{n}^{\prime}\right\}\right)\right)
\end{aligned}
$$

where we use the hatted variables

$$
\left\|\hat{\tilde{\lambda}}_{1}(t) \rrbracket=\right\| \tilde{\lambda}_{1} \rrbracket+t\left\|\tilde{\lambda}_{n} \rrbracket \quad\right\| \hat{\tilde{\lambda}}_{j}(t) \rrbracket=\| \tilde{\lambda}_{j} \rrbracket \quad \text { for } j \neq 1
$$


in the transformation of the external states in $A_{L}$. It makes no difference whether we write $\| \tilde{\hat{\lambda}}_{k} \rrbracket$ or simply $\| \tilde{\lambda}_{k} \rrbracket$ for the variables in $A_{R}$, since the shifts in $A_{R}$ do not involve these variables. However, as indicated in (4.14), we must account for the shift $|n\rangle \rightarrow|n\rangle-t|1\rangle$ explicitly. The change in sign between the Fourier transform of the internal state in $A_{L}$ compared to $A_{R}$ accounts for the fact that $\ell$ is the momentum flowing out of $A_{L}$ and in to $A_{R}$. Note that the supertwistors associated to the internal state have the same unprimed spinor part $\lambda_{A}$ in both $\mathcal{A}_{L}$ and $\mathcal{A}_{R}$.

We now insert these sub-amplitudes into (4.9) and transform the whole expression back to twistor space. For all the external states except 1 and $n$, this is trivial. Changing variables $\tilde{\lambda}_{1} \rightarrow \hat{\tilde{\lambda}}_{1}$ also allows us to perform the $\mathrm{d}^{2 \mid \mathcal{N}} \hat{\tilde{\lambda}}_{1} \mathrm{~d}^{2 \mid \mathcal{N}} \mu_{1}^{\prime}$ integrals directly. The remaining integrals are

$$
\begin{gathered}
\frac{1}{(2 \pi)^{6}} \int \frac{\mathrm{d} t}{t}\langle\lambda \mathrm{d} \lambda\rangle \mathrm{d}^{2 \mid \mathcal{N}} \mu \mathrm{d}^{2 \mid \mathcal{N}} \tilde{\lambda} \mathrm{d}^{2 \mid \mathcal{N}} \tilde{\lambda}_{n} \mathrm{~d}^{2 \mid \mathcal{N}} \mu^{\prime} \mathrm{d}^{2 \mid \mathcal{N}} \mu_{n}^{\prime} \mathrm{e}^{\mathrm{i} \llbracket\left(\mu-\mu^{\prime}\right) \tilde{\lambda} \rrbracket} \mathrm{e}^{\mathrm{i} \llbracket\left(\mu_{n}-\mu_{n}^{\prime}-t \mu_{1}\right) \tilde{\lambda}_{n} \rrbracket} \\
\times \operatorname{sgn}(\langle 1 \lambda\rangle[\tilde{\lambda} n]) \mathcal{A}_{L}\left(\mathrm{~W}_{1}, \ldots, \mathrm{W}\right) \mathcal{A}_{R}\left(\left\{\lambda, \mu^{\prime}, \chi^{\prime}\right\}, \ldots,\left\{\lambda_{n}-t \lambda_{1}, \mu_{n}^{\prime}, \chi_{n}^{\prime}\right\}\right) \\
=\frac{1}{(2 \pi)^{6}} \int \frac{\mathrm{d} t}{t} D^{3 \mid \mathcal{N}} \mathrm{W} \mathrm{d}^{2 \mid \mathcal{N}} \tilde{\lambda} \mathrm{d}^{2 \mid \mathcal{N}} \tilde{\lambda}_{n} \mathrm{~d}^{2 \mid \mathcal{N}} \mu^{\prime} \mathrm{d}^{2 \mid \mathcal{N}} \mu_{n}^{\prime} \mathrm{e}^{\mathrm{i} \llbracket\left(\mu-\mu^{\prime}\right) \tilde{\lambda} \rrbracket} \mathrm{e}^{\mathrm{i} \llbracket\left(\mu_{n}-\mu_{n}^{\prime}\right) \tilde{\lambda}_{n} \rrbracket} \\
\times \operatorname{sgn}(\langle 1 \lambda\rangle[\tilde{\lambda} n]) \mathcal{A}_{L}\left(\mathrm{~W}_{1}, \ldots, \mathrm{W}\right) \mathcal{A}_{R}\left(\mathrm{~W}^{\prime}, \ldots, \mathrm{W}_{n}^{\prime}-t \mathrm{~W}_{1}\right),
\end{gathered}
$$

where in the second line we have translated $\| \mu_{n}^{\prime} \rrbracket$ to $\left\|\mu_{n}^{\prime} \rrbracket-t\right\| \mu_{1} \rrbracket$ and defined

$$
\mathrm{W}^{\prime}:=\left(\lambda, \mu^{\prime}, \chi^{\prime}\right) \quad \text { and } \quad \mathrm{W}_{n}^{\prime}:=\left(\lambda_{n}, \mu_{n}^{\prime}, \chi_{n}^{\prime}\right) .
$$

We also combined $\langle\lambda \mathrm{d} \lambda\rangle \mathrm{d}^{2 \mid \mathcal{N}} \mu$ into the measure $D^{3 \mid \mathcal{N}} \mathrm{W}$ on the supertwistor space of the internal state.

To proceed, we somewhat formally write

$$
\operatorname{sgn}\left[\tilde{\lambda} \tilde{\lambda}_{n}\right]=\operatorname{sgn}\left[\frac{\partial}{\partial \mu^{\prime}} \frac{\partial}{\partial \mu_{n}^{\prime}}\right]
$$

inside the integrals. ${ }^{17}$ The operator $\operatorname{sgn}\left[\frac{\partial}{\partial \mu^{\prime}} \frac{\partial}{\partial \mu_{n}^{\prime}}\right]$ then acts on $\mathcal{A}_{R}$ (as a distribution), whereupon the remaining integrals (except those over $t$ and the internal supertwistor $\mathrm{W}$ ) become straightforward. We are left with

$$
\begin{aligned}
\mathcal{A}\left(\mathrm{W}_{1}, \ldots, \mathrm{W}_{n}\right)=\sum \int \frac{\mathrm{d} t}{t} D^{3 \mid \mathcal{N}} \mathrm{W} \mathcal{A}_{L}\left(\mathrm{~W}_{1}, \ldots, \mathrm{W}\right) \\
\times \operatorname{sgn}\left(\langle 1 \lambda\rangle\left[\frac{\partial}{\partial \mu} \frac{\partial}{\partial \mu_{n}}\right]\right) \mathcal{A}_{R}\left(\mathrm{~W}, \ldots, \mathrm{W}_{n}-t \mathrm{~W}_{1}\right) .
\end{aligned}
$$

The only $t$-dependence is inside $\mathcal{A}_{R}$. Since our split signature i $\epsilon$-prescription means that the $\mathrm{d} t / t$ integral to be understood as a principal value integral, from section 3.1.1 we can write

$$
\int \frac{\mathrm{d} t}{t} \mathcal{A}_{R}\left(\mathrm{~W}, \ldots, \mathrm{W}_{n}-t \mathrm{~W}_{1}\right)=\mathrm{i} \pi \operatorname{sgn}\left(\mathrm{iW} \cdot \frac{\partial}{\partial \mathrm{W}_{n}}\right) \mathcal{A}_{R}\left(\mathrm{~W}, \ldots, \mathrm{W}_{n}\right) .
$$

\footnotetext{
${ }^{17}$ The definition of this operator will always be via the Fourier transform. In particular, this makes transparent that $\operatorname{sgn}\left(\left[\partial_{i} \partial_{j}\right]\left[\partial_{i} \partial_{j}\right]\right)=1$, which will be a key property in manipulating the recursion relations
} 
Combining this with (4.19), we arrive at our final form of the BCFW recursion relation in (dual) supertwistor space:

$$
\begin{aligned}
\mathcal{A}\left(\mathrm{W}_{1}, \ldots, \mathrm{W}_{n}\right)=\sum & \int D^{3 \mid \mathcal{N}} \mathrm{W} \mathcal{A}_{L}\left(\mathrm{~W}_{1}, \ldots, \mathrm{W}\right) \\
& \times \mathrm{i} \pi \operatorname{sgn}\left(\left\langle\mathrm{W}_{1} \mathrm{~W}\right\rangle \mathrm{i} \mathrm{W}_{1} \cdot \frac{\partial}{\partial \mathrm{W}_{n}}\left[\frac{\partial}{\partial \mathrm{W}} \frac{\partial}{\partial \mathrm{W}_{n}}\right]\right) \mathcal{A}_{R}\left(\mathrm{~W}, \ldots, \mathrm{W}_{n}\right)
\end{aligned}
$$

where $\left\langle\mathrm{W}_{1} \mathrm{~W}_{2}\right\rangle=\langle 12\rangle$ and $\left[\partial_{\mathrm{W}} \partial_{\mathrm{W}_{n}}\right]=\partial^{2} / \partial \mu^{A^{\prime}} \partial \mu_{A^{\prime} n}$. Thus, in twistor space, BCFW recursion recursion involves gluing two sub-amplitudes together using the operator

$$
\operatorname{sgn}\left(\left\langle\mathrm{W}_{1} \mathrm{~W}\right\rangle \mathrm{W}_{1} \cdot \frac{\partial}{\partial \mathrm{W}_{n}}\left[\frac{\partial}{\partial \mathrm{W}} \frac{\partial}{\partial \mathrm{W}_{n}}\right]\right)
$$

and then integrating over the location of the intermediate supertwistor. Based on (4.21) and the forms of the three-point amplitudes given in (3.34), we immediately conclude that the complete classical S-matrix of $\mathcal{N}=4 \mathrm{SYM}$ can be written as a sum of products of sign operators acting on basic $\delta$-functions, with these products then integrated over some number of copies of supertwistor space.

Although the non-local operator (4.22) and integrals over internal twistors seem rather awkward and may initially seem disappointing, just as the momentum $\delta$-functions allow us to perform the integrals in (4.9) and return to the unintegrated form (4.1), we will see in the examples below that the recursion relation (4.21) is quite tractable and the integrals and operators can often be evaluated explicitly, although not yet so systematically as in the momentum space representation.

\section{SYM twistor amplitudes from BCFW recursion}

In this section we use the BCFW recursion relations to calculate the twistor form of various scattering amplitudes in $\mathcal{N}=4 \mathrm{SYM}$. We denote the $n$-particle $\mathrm{N}^{k} \mathrm{MHV}$ twistor superamplitude by $\mathcal{A}_{k}^{n}(1, \ldots, n)$, although we occasionally omit the super- or subscript when the context makes it clear.

\subsection{On the general structure of $\mathcal{N}=4$ amplitudes}

Scattering amplitudes in a theory with unbroken supersymmetry such as $\mathcal{N}=4$ SYM can only depend on combinations of Grassmann variables that are invariant under the Rsymmetry group. In split signature space-time this is $\operatorname{SL}(\mathcal{N} ; \mathbb{R})$, so invariants can only be constructed by complete contractions with the $\mathcal{N}$-dimensional Levi-Civita symbol. Thus, decomposing momentum space amplitudes into their homogeneity in the $\eta \mathrm{s}$, only multiples of $\mathcal{N}$ will appear for the homogeneities, with $\mathrm{N}^{k} \mathrm{MHV}$ amplitudes being homogeneous polynomials of degree $(k+2) \mathcal{N}$ in the $\eta \mathrm{s}$. The $n$-fold half Fourier transform for an $n$ particle amplitude sends such a homogeneous polynomial to a homogeneous polynomial of degree $(n-k-2) \mathcal{N}$ in the $\chi_{\mathrm{s}}$, so $n$-particle $\mathrm{N}^{k} \mathrm{MHV}$ amplitudes on (dual) supertwistor space have homogeneity $(n-k-2) \mathcal{N}$ in the anti-commuting variables. According to this 
counting, the 3-particle $\overline{\mathrm{MHV}}$ amplitude should be taken as having $k=-1$, but for all other tree amplitudes, $k \geq 0$.

We can use the recursion relations to show that, ignoring the conformal breaking sign factors, a general $n$-particle $\mathrm{N}^{k} \mathrm{MHV}$ super-amplitude is obtained by acting on $(n-2-$ k) $\delta^{(4 \mid 4)}$-functions with $2(n-2)$ Hilbert transforms. To start the induction, recall from section 3.2 the 3 -point $\mathrm{MHV}$ and $\overline{\mathrm{MHV}}$ amplitudes

$$
\begin{aligned}
\mathcal{A}_{0}^{3}(1,2,3) & =\operatorname{sgn}\langle 23\rangle \int \frac{\mathrm{d} s}{s} \frac{\mathrm{d} t}{t} \delta^{(4 \mid 4)}\left(\mathrm{W}_{1}-s \mathrm{~W}_{2}-t \mathrm{~W}_{3}\right) \\
\mathcal{A}_{-1}^{3}(1,2,3) & =\operatorname{sgn}\left[\partial_{2} \partial_{3}\right] \int \frac{\mathrm{d} s}{s} \frac{\mathrm{d} t}{t} \delta^{(4 \mid 4)}\left(\mathrm{W}_{2}-\mathrm{W}_{1}\right) \delta^{(4 \mid 4)}\left(\mathrm{W}_{3}-t \mathrm{~W}_{1}\right) ;
\end{aligned}
$$

these are constructed from two $(=2(n-2))$ Hilbert transforms acting on a product of $(1-k) \delta^{(4 \mid 4)}$-functions, where $k=0,-1$ for the MHV and $\overline{\mathrm{MHV}}$ amplitudes, respectively. Now proceed by induction on $n$ and $k$ : Suppose that a given term in the BCFW recursion decomposes an $n$-point $\mathrm{N}^{k} \mathrm{MHV}$ amplitude $\mathcal{A}_{k}^{n}$ into an $r$-point $\mathrm{N}^{l} \mathrm{MHV}$ amplitude $\mathcal{A}_{l}^{r}$ and a $s$-point $\mathrm{N}^{m} \mathrm{MHV}$ amplitude $\mathcal{A}_{m}^{s}$. Then

$$
n=r+s-2 \quad \text { and } \quad k=l+m+1 .
$$

The $\mathrm{D}^{3 \mid 4} W$ integration in the recursion removes one projective $\delta^{(3 \mid 4)}$-function, and this $\delta^{(3 \mid 4)}$-function is a single Hilbert transform of a $\delta^{(4 \mid 4)}$-function. Thus the total number of constituent $\delta^{(4 \mid 4)}$-functions in $\mathcal{A}_{k}^{n}$ is one less than the sum of the numbers in $\mathcal{A}_{l}^{r}$ and $\mathcal{A}_{m}^{2}$, i.e.

$$
\#\left(\delta^{(4 \mid 4)} \text {-functions }\right)=(r-2-l)+(s-2-m)=(n-2-k)
$$

by induction from the three-point amplitudes. On the other hand, the gluing operator (4.22) itself involves the Hilbert transform $\operatorname{sgnW}_{1} \cdot \partial_{n}$ (which cannot cancel with one in the right hand amplitude as $\mathrm{W}_{1}$ is not a variable in that amplitude), so the net number of Hilbert transforms remains the same and we inductively find

$$
\text { \# (Hilbert transforms })=2(r-2)+2(s-2)=2(n-2)
$$

as was to be proved.

The other constituents in the 3-point amplitudes and the recursion relations are the local and non-local sign factors $\operatorname{sgn}\langle i j\rangle$ and $\operatorname{sgn}\left[\partial_{i} \partial_{j}\right]$. It is clear from the form of the discussion of conformal properties of the $n$-point MHV amplitudes in section 3.3 that there is ample scope for the cancellation of these factors so we can make no uniform statement about how many of these survive in a final formula for an amplitude.

\subsection{Solving the recursion relations}

There are two terms in the BCFW decomposition of a generic amplitude $\mathcal{A}_{k}^{n}$ that play a somewhat distinguished role - when one or other of the two sub-amplitudes is a threepoint amplitude. In these cases, momentum space kinematics ensure that with the $[1 n\rangle$ shift we have chosen, only the right sub-amplitude can be $\overline{M H V}$ (in which case the left sub-amplitude is $\mathcal{A}_{k}^{n-1}$ ), while only the left sub-amplitude can be MHV (in which case 


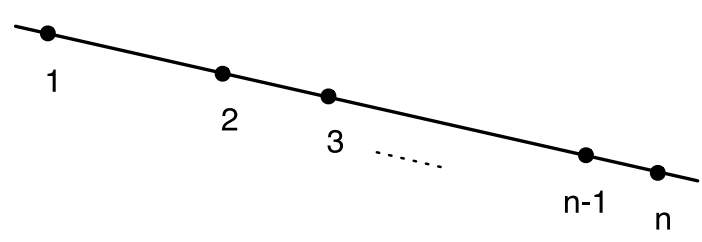

Figure 1. The MHV amplitude is supported on a line in twistor space.

the right sub-amplitude is $\mathcal{A}_{k-1}^{n-1}$ ). We call the first case the 'homogeneous contribution' following [35], and the second case the 'conjugate homogeneous contribution'. We now explain how to perform the integral in the twistor BCFW recursion explicitly in these two cases. This will form the basis of our strategy for solving the recursion relations in general. The outcome of these recursions simply leads to the action of operators $\mathcal{H}_{i-1, i+1}^{i} \delta^{4 \mid 4}\left(W_{i}\right)$ and $\widetilde{\mathcal{H}}_{i}^{i-1, i+1}$ on the remaining sub-amplitudes that insert particle $i$ inbetween $i-1$ and $i+1$ in the remaining subamplitude. These operators can be identified with the inverse soft limits of [46].

\subsubsection{The homogeneous term and MHV amplitudes}

The homogeneous contribution to the twistor BCFW decomposition of $\mathcal{A}_{n}^{k}$ is

$$
\int D^{3 \mid 4} \mathrm{~W} \mathcal{A}_{k}(1, \ldots, n-2, \mathrm{~W}) \operatorname{sgn}\left(\langle 1 \mathrm{~W}\rangle \mathrm{iW}_{1} \cdot \partial_{n}\left[\partial_{\mathrm{W}} \partial_{n}\right]\right) \mathcal{A}_{-1}(\mathrm{~W}, n-1, n) .
$$

Recalling the form (5.1) of the $\overline{\mathrm{MHV}}$ amplitude we see that the $\operatorname{sgn}\left[\partial_{\mathrm{W}} \partial_{n}\right]$ operators in the sub-amplitude and recursion cancel up to a constant factor of -1 , coming from the different ordering of $\mathrm{W}$ and $\mathrm{W}_{n}$ in the two terms. Since

$$
\mathrm{i} \pi \operatorname{sgn}\left(\mathrm{iW} \cdot \partial_{n-1}\right) \delta^{(4 \mid 4)}\left(\mathrm{W}_{n-1}\right)=\tilde{\delta}^{(3 \mid 4)}\left(\mathrm{W}_{n-1}, \mathrm{~W}\right)
$$

we can perform the $D^{3 \mid 4} \mathrm{~W}$ integration trivially, yielding the contribution

$$
\mathcal{A}_{k}(1, \ldots, n-2, n-1) \times \operatorname{sgn}\left(\langle 1 n-1\rangle \mathrm{iW}_{1} \cdot \partial_{n} \mathrm{iW}_{n-1} \cdot \partial_{n}\right) \delta^{(4 \mid 4)}\left(\mathrm{W}_{n}\right) .
$$

Recognising the 3-point MHV amplitude, we obtain the final form

$$
-\mathcal{A}_{k}(1, \ldots, n-2, n-1) \times \mathcal{A}_{0}(1, n, n-1)
$$

for the contribution to $\mathcal{A}_{k}^{n}$ from the homogeneous term. Thus the homogeneous term simply tacks on a 3 -point MHV amplitude to the $(n-1)$-particle $\mathrm{N}^{k} \mathrm{MHV}$ amplitude. This has the effect of inserting the dual twistor $\mathrm{W}_{n}$ in between $\mathrm{W}_{n-1}$ and $\mathrm{W}_{1}$, that were adjacent in the sub-amplitude.

For MHV amplitudes $(k=0)$, the homogeneous term is the complete BCFW decomposition and we immediately obtain

$$
\begin{aligned}
\mathcal{A}_{0}^{n}(1, \ldots, n) & =(-1)^{n-3} \prod_{i=3}^{n} \mathcal{A}_{0}(1, i-1, i) \\
& =\prod_{i=3}^{n} \mathcal{H}_{i-1, i-2}^{i} \delta^{(4 \mid 4)}\left(\mathrm{W}_{i}\right)
\end{aligned}
$$


in agreement with equation (3.29). The basic three point MHV amplitude is proportional to a collinear delta function, so the $n$-particle MHV amplitude requires that all the points are collinear in twistor space, as is well-known. (See figure 1.)

\subsubsection{The conjugate homogeneous term and the $\overline{M H V}$ amplitudes}

The conjugate homogeneous contribution to the decomposition of $\mathcal{A}_{k}^{n}$ is

$$
\int \mathrm{D}^{3 \mid 4} \mathrm{~W} \mathcal{A}_{0}(1,2, \mathrm{~W}) \operatorname{sgn}\left(\langle 1 \mathrm{~W}\rangle \mathrm{iW}_{1} \cdot \partial_{n}\left[\partial_{\mathrm{W}} \partial_{n}\right]\right) \mathcal{A}_{k-1}^{n-1}(\mathrm{~W}, 3, \ldots, n) .
$$

From (5.1) we have

$$
\mathcal{A}_{0}(1,2, \mathrm{~W})=\mathrm{i} \pi \operatorname{sgn}\left(\langle\mathrm{W} 1\rangle \mathrm{iW}_{1} \cdot \frac{\partial}{\partial \mathrm{W}_{2}}\right) \tilde{\delta}^{(3 \mid 4)}\left(\mathrm{W}_{2}-s \mathrm{~W}_{1}, \mathrm{~W}\right)
$$

and the $\tilde{\delta}^{(3 \mid 4)}$-function again allows us to perform the $D^{3 \mid 4} \mathrm{~W}$ integral directly. We obtain

$$
-\pi^{2} \operatorname{sgn}\left(\left[\partial_{2} \partial_{n}\right] \mathrm{iW}_{1} \cdot \partial_{2} \mathrm{iW}_{1} \cdot \partial_{n}\right) \mathcal{A}_{k-1}^{n-1}(2,3, \ldots, n)=-\widetilde{\mathcal{H}}_{1}^{2 n} \mathcal{A}_{k-1}^{n-1}(2,3, \ldots, n) .
$$

Applying $\widetilde{\mathcal{H}}_{1}^{2 n}$ to $\mathcal{A}_{k-1}^{n-1}(2, \ldots, n)$ inserts the point $\mathrm{W}_{1}$ in between $\mathrm{W}_{2}$ and $\mathrm{W}_{n}$, which are adjacent in the colour ordering of the sub-amplitude.

Just as the homogeneous term is the only contribution to the BCFW decomposition of an MHV amplitude, so too this conjugate homogeneous term is the only contribution to the 'googly MHV' amplitude $\mathcal{A}_{n-4}^{n}$ - the CPT conjugates of the MHV amplitudes. To see this, first note that for $\mathcal{A}_{k}^{n} \neq 0$, generically $k \leq n-4$ (with the equality holding for the googly MHV amplitudes) except that the three-point MHV amplitude has $k=n-3$. This CPT conjugate to the statement that, with the exception of the 3-point $\overline{\mathrm{MHV}}$ amplitude $\mathcal{A}_{-1}^{3}$, amplitudes with $k<0$ vanish. Now, if we decompose a googly MHV amplitude $\mathcal{A}_{n-4}^{n}$ into $\mathcal{A}_{l}^{r}$ and $\mathcal{A}_{m}^{s}$ sub-amplitudes, since $r+s=n+2$ and $l+m=n-4+1$ we must have $(r-l)+(s-m)=7$. Consequently, one of the sub-amplitudes must be a three-point MHV and momentum kinematics dictates that it is the left sub-amplitude. The other subamplitude is then $\mathcal{A}_{n-5}^{n-1}$; the googly MHV amplitude with one fewer leg. Thus (5.12) is the only contribution to the BCFW decomposition of a googly MHV amplitude. Continuing recursively we have

$$
\begin{aligned}
\mathcal{A}_{n-4}^{n}(1, \ldots, n) & =(-1)^{n}\left(\prod_{i=2}^{n-1} \widetilde{\mathcal{H}}_{i-1}^{i n}\right) \delta^{(4 \mid 4)}\left(\mathrm{W}_{n-1}\right) \delta^{(4 \mid 4)}\left(\mathrm{W}_{n}\right) \\
& =\left(\prod_{i=2}^{n-3} \simeq \mathcal{H}_{i-1}^{i n}\right) \mathcal{A}_{0}^{4}(n-3, n-2, n-1, n) .
\end{aligned}
$$

In this expression, the $\widetilde{\mathcal{H}}_{i-1}^{i n}$ do not commute and are ordered with increasing $i$ to the right. To perform the last step of the induction we used the specific form of the 3-point $\overline{\mathrm{MHV}}$ amplitude. Thus the googly MHV amplitudes are built up from a product of $\widetilde{\mathcal{H}}$ operators acting on two $\delta^{(4 \mid 4)}$-functions.

Cyclic symmetry of the googly MHV amplitudes implies many identities in these formulæ that are not manifest, but which will be useful in the following. In particular, there 
is an obvious relation from the cyclic symmetry of the 3-point amplitude, while that of the four-point amplitude yields

$$
\widetilde{\mathcal{H}}_{1}^{24} \widetilde{\mathcal{H}}_{2}^{34} \delta^{(4 \mid 4)}\left(\mathrm{W}_{3}\right) \delta^{(4 \mid 4)}\left(\mathrm{W}_{4}\right)=\widetilde{\mathcal{H}}_{2}^{31} \widetilde{\mathcal{H}}_{3}^{41} \delta^{(4 \mid 4)}\left(\mathrm{W}_{4}\right) \delta^{(4 \mid 4)}\left(\mathrm{W}_{1}\right)
$$

which is the CPT conjugate of the relation (3.38) among the $\mathcal{H}_{j k}^{i}$ operators. Finally, since the four-point amplitude may be represented either as an MHV or a googly MHV amplitude, we have

$$
\mathcal{H}_{13}^{2} \mathcal{H}_{13}^{4} \delta^{(4 \mid 4)}\left(\mathrm{W}_{2}\right) \delta^{(4 \mid 4)}\left(\mathrm{W}_{4}\right)=\widetilde{\mathcal{H}}_{1}^{24} \widetilde{\mathcal{H}}_{2}^{34} \delta^{(4 \mid 4)}\left(\mathrm{W}_{3}\right) \delta^{(4 \mid 4)}\left(\mathrm{W}_{4}\right) .
$$

As with the MHV amplitudes, we can use the cyclic identities to ensure that the $\operatorname{sgn}\left[\partial_{i} \partial_{j}\right]$ factors cancel pairwise, leaving us with at most one such factor. Thus, following the discussion of section 3.3, the $\overline{\mathrm{MHV}}$ amplitudes with an odd number of external particles violate conformal invariance - they do not extend to the conformal compactification of affine spacetime, but rather to its double cover.

\subsection{NMHV amplitudes}

We now compute the twistor form of the NMHV amplitude $\mathcal{A}_{1}^{n}$. For $n=5$ this is a googly MHV and (5.13) gives

$$
\begin{aligned}
\mathcal{A}_{1}^{5}(1,2,3,4,5) & =\widetilde{\mathcal{H}}_{1}^{25} \widetilde{\mathcal{H}}_{2}^{35} \widetilde{\mathcal{H}}_{3}^{45} \delta^{(4 \mid 4)}\left(\mathrm{W}_{4}\right) \delta^{(4 \mid 4)}\left(\mathrm{W}_{5}\right) \\
& =-\widetilde{\mathcal{H}}_{1}^{25} \mathcal{A}_{0}^{4}(2,3,4,5) .
\end{aligned}
$$

In fact, we will be able to write the general $n$-particle NMHV amplitude in terms of this 5 -point amplitude and $m$-point MHV amplitudes. To see this, note that the contribution to an $n$-point NMHV amplitude from all but the homogeneous term is

$$
\sum_{i=2}^{n-3} \int D^{3 \mid 4} \mathrm{~W} \mathcal{A}_{0}^{i+1}(1, \ldots, i, \mathrm{~W}) \operatorname{sgn}\left(\langle 1 \mathrm{~W}\rangle \mathrm{iW}_{1} \cdot \partial_{n}\left[\partial_{\mathrm{W}} \partial_{n}\right]\right) \mathcal{A}_{0}^{n-i+1}(\mathrm{~W}, i+1, \ldots, n) .
$$

Using (3.29) (or (5.9)) we can split the MHV sub-amplitudes as ${ }^{18}$

$$
\begin{aligned}
\mathcal{A}_{0}^{i+1}(1, \ldots, i, \mathrm{~W}) & =-\mathcal{A}_{0}^{i}(1, \ldots, i) \mathcal{A}_{0}(1, i, \mathrm{~W}) \\
\mathcal{A}_{0}^{n-i+1}(\mathrm{~W}, i+1, \ldots, n) & =\mathcal{A}_{0}^{n-i-1}(i+1, \ldots, n-1) \mathcal{A}_{0}^{4}(i+1, n-1, n, \mathrm{~W}) .
\end{aligned}
$$

The first terms on the rhs of these equations are independent of both $\mathrm{W}$ and $\mathrm{W}_{n}$, so may be brought outside both the $D^{3 \mid 4} \mathrm{~W}$ integral and the non-local sign operators in (5.17). Thus we only need consider the expression

$$
\int D^{3 \mid 4} \mathrm{~W} \mathcal{A}_{0}(1, i, \mathrm{~W}) \times \operatorname{sgn}\left(\langle 1 \mathrm{~W}\rangle \mathrm{iW}_{1} \cdot \partial_{n}\left[\partial_{\mathrm{W}} \partial_{n}\right]\right) \mathcal{A}_{0}^{4}(i+1, n-1, n, \mathrm{~W}) .
$$

But this is conjugate homogeneous and is just the five-point NMHV super-amplitude $\mathcal{A}_{1}^{5}(1, i, i+1, n-1, n)$ ! Therefore, the sum of contributions (5.17) reduces to ${ }^{19}$

$$
\sum_{i=2}^{n-3} \mathcal{A}_{0}^{i}(1, \ldots, i) \mathcal{A}_{1}^{5}(1, i, i+1, n-1, n) \mathcal{A}_{0}^{n-i-1}(i+1, \ldots, n-1),
$$

\footnotetext{
${ }^{18}$ When $i=2$ or $n-3$ no splitting is necessary.

${ }^{19}$ When $i=2$ or $i=n-3$, the 'two-point' MHV amplitudes in this sum should be replaced by unity.
} 
while the homogeneous term is $\mathcal{A}_{1}^{n-1}(1, \ldots, n-1) \mathcal{A}_{0}^{3}(1, n, n-1)$.

Working by induction, one can show that this recursive formula is solved by the double sum

$$
\begin{aligned}
\mathcal{A}_{1}(1, \ldots, n)=\sum_{j=5}^{n} \sum_{i=2}^{j-3} \mathcal{A}_{1}^{5}(1, i, i+1, j-1, j) \\
\quad \times \mathcal{A}_{0}(1, \ldots, i) \mathcal{A}_{0}(i+1, \ldots, j-1) \mathcal{A}_{0}(j, \ldots, n, 1)
\end{aligned}
$$

where all 'two-point $\mathcal{A}_{0}$ amplitudes' should be replaced by unity. For example, the twistor form of the 6-particle NMHV amplitude equals

$$
\mathcal{A}_{1}(1,2,3,4,5) \mathcal{A}_{0}(1,5,6)+\mathcal{A}_{1}(1,2,3,5,6) \mathcal{A}_{0}(3,4,5)+\mathcal{A}_{1}(1,3,4,5,6) \mathcal{A}_{0}(1,2,3) .
$$

Notice that the $n$-particle MHV amplitude may be decomposed as

$$
\mathcal{A}_{0}(1, i, i+1, j-1, j) \times \mathcal{A}_{0}(1, \ldots, i) \mathcal{A}_{0}(i+1, \ldots, j-1) \mathcal{A}_{0}(j, \ldots, n, 1)
$$

whenever $i$ and $j$ lie in the ranges permitted by the double sum in (5.21). Thus, to obtain an NMHV amplitude from the MHV amplitude, one chooses two points $\left(\mathrm{W}_{i}, \mathrm{~W}_{j}\right)$ with $i-j>2$, and replaces the five-point MHV amplitude

$$
\begin{aligned}
\mathcal{A}_{0}(1, i, i+1, j-1, j) & =-\mathcal{A}_{0}(1, i, j) \mathcal{A}_{0}(i, i+1, j-1, j) \\
& =-\mathcal{H}_{i j}^{1} \delta^{(4 \mid 4)}\left(\mathrm{W}_{1}\right) \mathcal{A}_{0}(i, i+1, j-1, j)
\end{aligned}
$$

by the five-point NMHV amplitude

$$
\mathcal{A}_{1}(1, i, i+1, j-1, j)=-\widetilde{\mathcal{H}}_{1}^{i j} \mathcal{A}_{0}(i, i+1, j-1, j) .
$$

The twistor $\mathrm{W}_{1}$ is distinguished here purely through its role in the BCFW recursion relation. Put simply, one organises the amplitude so that $\mathcal{A}_{0}(1, i, j)$ is explicit, and replaces it with an $\widetilde{\mathcal{H}}_{1}^{i j}$. This replacement is perhaps most analogous to multiplication of an MHV amplitude by the first of the dual superconformal invariants $R$ (or $\mathcal{P}$ ) in the work of Drummond et al. [14, 33, 35]. Although these invariants are local on momentum space, they becomes non-local on twistor space via the half Fourier transform. (We discuss the analogues of the higher $R$ invariants of [35] later.)

Geometrically, the twistor support of the NMHV amplitude can be understood, provided we ignore the effect of the nonlocal operator $\operatorname{sgn}\left(\left[\partial_{i} \partial_{j}\right]\right)$ in $\widetilde{\mathcal{H}}_{1}^{i j}$. (This operator is \pm 1 on momentum space, so goes unnoticed if one tries to determine the localisation properties by acting on the momentum space amplitudes with certain differential operators as in [1].) Replacing this operator by 1 , we find

$$
\begin{aligned}
& \mathcal{A}_{1}(1, i, i+1, j-1, j) \rightarrow(\mathrm{i} \pi)^{2} \operatorname{sgn}\left(\mathrm{iW}_{1} \cdot \partial_{i} \mathrm{iW}_{1} \cdot \partial_{j}\right) \mathcal{A}_{0}(i, i+1, j-1) \mathcal{A}_{0}(i, j-1, j) \\
& \quad=\int \frac{\mathrm{d} s}{s} \frac{\mathrm{d} t}{t} \mathcal{A}_{0}\left(\mathrm{~W}_{i}-s \mathrm{~W}_{1}, \mathrm{~W}_{i+1}, \mathrm{~W}_{j-1}\right) \mathcal{A}_{0}\left(\mathrm{~W}_{i}-s \mathrm{~W}_{1}, \mathrm{~W}_{j-1}, \mathrm{~W}_{j}-t \mathrm{~W}_{1}\right)
\end{aligned}
$$

This is a product of coplanar delta functions that altogether impose the condition that $\mathrm{W}_{1}, \mathrm{~W}_{i}, \mathrm{~W}_{i+1}, \mathrm{~W}_{j-1}$ and $\mathrm{W}_{j}$ are all coplanar. The remaining MHV amplitudes in (5.21) 


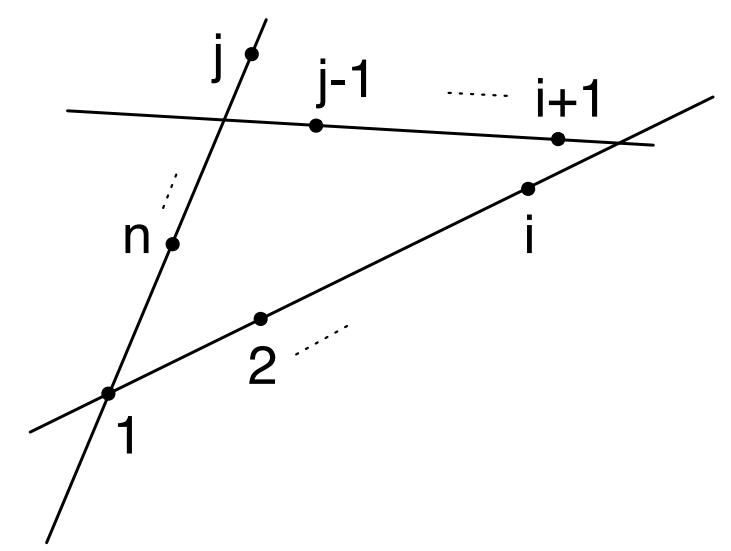

Figure 2. The NMHV amplitude is supported on three coplanar lines in twistor space. Point 1 (distinguished by its role in the BCFW recursion relations) is located at the intersection of two of these lines.

then require that the MHV amplitudes $\mathcal{A}_{0}(1, \ldots, i), \mathcal{A}_{0}(i+1, \ldots, j-1)$ and $\mathcal{A}_{0}(j, \ldots, n, 1)$ lie in this plane. Thus, ignoring the non-local $\operatorname{sgn}\left[\partial_{i} \partial_{j}\right]$ operator, the overall picture of an NMHV amplitude is shown in figure 2. This is initially at variance with the picture from twistor-string theory, in which the NMHV contribution should be based on a degree-two curve in the connected prescription, or a pair of skew lines in the disconnected prescription, rather than the three coplanar lines of figure 2. However, the five-point NMHV amplitude can be presented in various forms; in particular it may be represented as a sum of contributions that are supported on two intersecting lines by half Fourier transforming the MHV formalism. This then decomposes the above formula into a rather larger sum of terms supported on two lines. What is perhaps more interesting is that this configuration is precisely that discovered in [51] for the twistor space support of the 1-loop NMHV box coefficients, showing that the BCFW decomposition of a tree amplitude encodes its origin in terms of the soft limit of a one loop amplitude.

\subsection{Conjugate NMHV amplitudes}

The recursion for the $\mathcal{A}_{n-5}^{n}$ amplitudes is essentially conjugate to that of the NMHV amplitudes. The conjugate homogeneous term involves a 3-point MHV sub-amplitude and a conjugate NMHV sub-amplitude with one fewer points; its contribution is

$$
-\widetilde{\mathcal{H}}_{1}^{2 n} \mathcal{A}_{n-6}^{n-1}(2, \ldots, n) .
$$

In the remaining terms, each sub-amplitude is googly MHV. These terms are given by

$$
\begin{gathered}
\int \mathrm{D}^{3 \mid 4} \mathrm{~W}\left(\prod_{j=2}^{i-2} \widetilde{\mathcal{H}}_{j+1}^{j i}\right) \mathcal{A}_{0}(1,2, i) \mathcal{A}_{0}(1, \mathrm{~W}, i) \operatorname{sgn}\left(\langle 1 \mathrm{~W}\rangle \mathrm{W}_{1} \cdot \partial_{n}\left[\partial_{\mathrm{W}} \partial_{n}\right]\right) \\
\times \mathcal{A}_{n-i-4}^{n-i}(\mathrm{~W}, i+1, \ldots, n),
\end{gathered}
$$

summed over $i$ for $i=2, \ldots, n-2$ and with the $\widetilde{\mathcal{H}}$-operators ordered with increasing $j$ to the left. (We have used the cyclic property of the $\overline{\mathrm{MHV}}$ amplitude to ensure that 1 and $\mathrm{W}$ 
appear together in a 3-point MHV amplitude and have furthermore used a reverse ordered form of (5.13) to represent the left hand google-MHV amplitude.) To perform the integral, use the delta-function in $\mathcal{A}_{0}(i, 1, \mathrm{~W})$ (as in the conjugate homogeneous term). We obtain

$$
\begin{aligned}
-\left(\prod_{j=2}^{i-2} \widetilde{\mathcal{H}}_{j+1}^{j i}\right) \mathcal{A}_{0}(1,2, i) \widetilde{\mathcal{H}}_{1}^{i n} \mathcal{A}_{n-i-4}^{n-i}(i, i+1, \ldots, n) \\
=\left(\prod_{j=2}^{i-2} \widetilde{\mathcal{H}}_{j+1}^{j i}\right) \mathcal{A}_{0}(1,2, i) \mathcal{A}_{n-i-3}^{n-i+1}(1, i, \ldots, n),
\end{aligned}
$$

where we have used (5.13) to identify $-\widetilde{\mathcal{H}}_{1}^{i n} \mathcal{A}_{n-i-4}^{n-i}(i, i+1, \ldots, n)$ as an $\overline{\mathrm{MHV}}$ amplitude.

Adding up the homogeneous and the inhomogeneous terms, one finds

$$
\mathcal{A}_{n-5}^{n}=\sum_{2 \leq i<j \leq n-2}\left(\prod_{l=2}^{i-1}-\widetilde{\mathcal{H}}_{l-1}^{l n}\right)\left(\prod_{m=i}^{j-2} \widetilde{\mathcal{H}}_{m+1}^{m j}\right) \mathcal{A}_{0}(i-1, i, j)\left(\prod_{r=j}^{n-2} \widetilde{\mathcal{H}}_{r+1}^{r, n-1}\right) \mathcal{A}_{0}(i, j, n-1, n),
$$

where the $\widetilde{\mathcal{H}}$-operators are ordered with increasing $m, r$ indices to the left, but $l$ increasing to the right and the product should be taken to be 1 if the top limit is smaller than the lower one. Crudely, this formula differs from the conjugate MHV amplitude by replacing an $\widetilde{\mathcal{H}}$-operator by a three-point $\mathcal{A}_{0}$ amplitude.

\subsection{Further tree examples}

Rather than give more closed-form formulae, we just describe the strategy for integrating the recursion relation and outline the structure of the terms that arise. The homogeneous or conjugate homogeneous term of any amplitude may be regarded as understood, at least inductively, via the discussion of section 5.2.

In a generic $\mathrm{N}^{2} \mathrm{MHV}$ amplitude, the remaining inhomogeneous terms involve one NMHV and one MHV sub-amplitude. If the MHV sub-amplitude is on the left, we can perform the integral over the internal twistor using the same strategy as for the conjugate homogeneous term. This yields a product of an MHV sub-amplitude and an $\widetilde{\mathcal{H}}$ operator acting on the NMHV sub-amplitude on the right. Conversely, suppose that the MHV sub-amplitude is on the right inside the integral, so that the NMHV sub-amplitude $\mathcal{A}_{1}^{r}(1, \ldots, r-1, \mathrm{~W})$ is on the left. The form (5.21) of the NMHV amplitude shows that either $\mathrm{W}$ appears only in an MHV sub-amplitude - in which case we can again proceed as in the conjugate homogeneous term - or else must appear in a five-point NMHV amplitude $\mathcal{A}_{1}^{5}(1, i, i+1, r-1, \mathrm{~W})$ for some $i$. Even in this case, the conjugate homogeneous strategy can be used: The cyclic symmetry of the 5-point NMHV amplitude ensures that it can always be written so that $\mathrm{W}$ is not acted on by $\widetilde{\mathcal{H}}$, e.g.

$$
\mathcal{A}_{1}(1, i, i+1, r-1, \mathrm{~W})=\widetilde{\mathcal{H}}_{i}^{i+1, r-1} \mathcal{A}_{0}(1, i+1, r-1, \mathrm{~W})
$$

Since $\widetilde{\mathcal{H}}$ is independent of $\mathrm{W}$ it may be brought outside to act on the result of the remaining integral, and this integral is of the same type as contributed to the NMHV amplitudes. 
In all cases we obtain two $\widetilde{\mathcal{H}}$-operators acting on a sequence of $\mathcal{A}_{0}$ factors, again demonstrating the similarity between the role of the $\widetilde{\mathcal{H}}$ operators and the dual superconformal invariants $R$ of $[14,33,35]$.

The 7 point $\mathrm{N}^{2} \mathrm{MHV}$ is the first non-trivial example of an $\overline{\mathrm{NMHV}}$ amplitude, and here (5.29) runs over six terms. The next case is the 8 point $\mathrm{N}^{2} \mathrm{MHV}$ with 20 terms:

$$
\begin{aligned}
& \widetilde{\mathcal{H}}_{1}^{28}\left(\mathcal{A}_{0}(2678) \widetilde{\mathcal{H}}_{2}^{36} \mathcal{A}_{0}(3456)+\mathcal{A}_{0}(278) \widetilde{\mathcal{H}}_{2}^{37} \mathcal{A}_{0}(34567)\right. \\
& +\widetilde{\mathcal{H}}_{2}^{38} \mathcal{A}_{0}(345678)+\mathcal{A}_{0}(234) \mathcal{A}_{0}(278) \widetilde{\mathcal{H}}_{2}^{47} \mathcal{A}_{0}(4567) \\
& \left.+\mathcal{A}_{0}(234) \widetilde{\mathcal{H}}_{2}^{48} \mathcal{A}_{0}(45678)+\mathcal{A}_{0}(2345) \widetilde{\mathcal{H}}_{2}^{58} \mathcal{A}_{0}(5678)\right) \\
& +\mathcal{A}_{0}(178)\left(\widetilde{\mathcal{H}}_{1}^{27} \mathcal{A}_{0}(267) \widetilde{\mathcal{H}}_{2}^{36} \mathcal{A}_{0}(3456)+\widetilde{\mathcal{H}}_{1}^{27} \mathcal{A}_{0}(456) \widetilde{\mathcal{H}}_{2}^{37} \mathcal{A}_{0}(3467)\right. \\
& +\widetilde{\mathcal{H}}_{1}^{27} \mathcal{A}_{0}(234) \widetilde{\mathcal{H}}_{2}^{47} \mathcal{A}_{0}(4567)+\mathcal{A}_{0}(167) \widetilde{\mathcal{H}}_{1}^{26} \widetilde{\mathcal{H}}_{2}^{36} \mathcal{A}_{0}(3456) \\
& \left.+\mathcal{A}_{0}(123) \widetilde{\mathcal{H}}_{1}^{37} \widetilde{\mathcal{H}}_{3}^{47} \mathcal{A}_{0}(4567)+\widetilde{\mathcal{H}}_{3}^{24} \mathcal{A}_{0}(124) \widetilde{\mathcal{H}}_{1}^{47} \mathcal{A}_{0}(4567)\right) \\
& +\mathcal{A}_{0}(123) \widetilde{\mathcal{H}}_{1}^{38} \mathcal{A}_{0}(378) \widetilde{\mathcal{H}}_{3}^{47} \mathcal{A}_{0}(4567)+\mathcal{A}_{0}(123) \widetilde{\mathcal{H}}_{1}^{38} \mathcal{A}_{0}(567) \widetilde{\mathcal{H}}_{3}^{48} \mathcal{A}_{0}(4578) \\
& +\mathcal{A}_{0}(123) \widetilde{\mathcal{H}}_{1}^{38} \mathcal{A}_{0}(345) \widetilde{\mathcal{H}}_{2}^{58} \mathcal{A}_{0}(5678)+\widetilde{\mathcal{H}}_{1}^{25} \mathcal{A}_{0}(2345) \widetilde{\mathcal{H}}_{1}^{58} \mathcal{A}_{0}(5678) \\
& +\mathcal{A}_{0}(345) \widetilde{\mathcal{H}}_{3}^{25} \mathcal{A}_{0}(125) \widetilde{\mathcal{H}}_{1}^{58} \mathcal{A}_{0}(5678)+\mathcal{A}_{0}(123) \widetilde{\mathcal{H}}_{4}^{35} \mathcal{A}_{0}(135) \widetilde{\mathcal{H}}_{1}^{58} \mathcal{A}_{0}(5678) \\
& +\mathcal{A}_{0}(1234) \widetilde{\mathcal{H}}_{1}^{48} \widetilde{\mathcal{H}}_{4}^{58} \mathcal{A}_{0}(5678)+\widetilde{\mathcal{H}}_{2}^{13} \mathcal{A}_{0}(134) \widetilde{\mathcal{H}}_{1}^{48} \mathcal{A}_{0}(45678) \text {. }
\end{aligned}
$$

In this equation all operators act on everything to their right within a term.

For $\overline{\mathrm{N}^{2} \mathrm{MHV}}$ amplitudes, the inhomogeneous terms involve sub-amplitudes that are either $\overline{\mathrm{MHV}}$ on the left and $\overline{\mathrm{NMHV}}$ on the right, or the other way around. The construction of the $\overline{\mathrm{NMHV}}$ amplitudes already showed how to perform the integration when the $\overline{\mathrm{MHV}}$ is on the left, i.e. via the conjugate homogeneous strategy. When the $\overline{\mathrm{MHV}}$ sub-amplitude $\mathcal{A}_{r-4}^{r}(\mathrm{~W}, n-r+2, \ldots, n)$ is on the right, use (5.13) to express it as a sequence of $(r-2) \widetilde{\mathcal{H}}$ operators acting on $\mathcal{A}_{-1}(n-1, \mathrm{~W}, n)$. Since these operators do not depend on $\mathrm{W}$ or $\mathrm{W}_{n}$, and can be chosen so as not to act on $\mathrm{W}$, they can be taken out of the integral. The remaining integral is then straightforward to perform using the standard, homogeneous strategy.

\subsection{General tree amplitudes}

In the examples above, it was convenient to express an $n$-point $\mathrm{N}^{k} \mathrm{MHV}$ amplitude in terms of $k \widetilde{\mathcal{H}}$-operators acting on $(n-2-k)$ three-point MHV sub-amplitudes in some order and it seems likely that it will be possible to express all tree amplitudes in this way. It is natural to ask for the correspondence with the dual superconformal invariants that arise in the scheme of Drummond et al. $[14,33,35]$. While we have no definitive answer, it seems likely that multiplication by the simplest dual superconformal invariant $R_{r ; s t}$ corresponds to a single insertion of an $\widetilde{\mathcal{H}}_{k}^{i j}$-operator. More complicated $R$-invariants arise when more than one $\widetilde{\mathcal{H}}$-operator is inserted (e.g. in $\mathrm{N}^{2} \mathrm{MHV}$ amplitudes) and we expect that their additional indices encode the ordering and nesting of the $\widetilde{\mathcal{H}}$-operators. 
Each of the $k \widetilde{\mathcal{H}}$-operators contains two Hilbert transforms and each of the $(n-2-k)$ three-point MHV amplitudes is an $\mathcal{H}$ operator (two Hilbert transforms) acting on a $\delta^{(4 \mid 4)_{-}}$ function. This gives a total of $2(n-2)$ Hilbert transforms acting on $(n-2-k) \delta^{(4 \mid 4)}$ functions, decorated by an unspecified number of $\operatorname{sgn}\left(\left\langle\mathrm{W}_{i} \mathrm{~W}_{j}\right\rangle\right)$ factors or $\operatorname{sgn}\left(\left[\partial_{i} \partial_{j}\right]\right)$ operators. These factors are constructed using the infinity twistor and, as with the three-point seed amplitudes, their presence indicates a violation of conformal invariance. We know that tree amplitudes in momentum space for $\mathcal{N}=4 \mathrm{SYM}$ are annihilated by the conformal algebra (at least away from their singularities), so this violation must again be rather subtle and indeed on momentum space these become locally constant functions and so their derivatives with respect to the conformal algebra vanish away from the singularities. A further subtlety is that there is ample opportunity for cancellation of the conformal-breaking signs, so the question as to whether their presence is essential in a given amplitude is not always clear.

In general, the existence of at least two $\delta^{(4 \mid 4)}$-functions in each amplitude means that there will always be more than one way to perform the $\mathrm{W}$-integration in the recursion, and the two strategies that we relied upon in our examples are no doubt not exhaustive. The arguments so far show that the internal state can in practice be integrated out to leave expressions in terms of $\tilde{\mathcal{H}}$ operators and three point MHV amplitudes, at least up to the $\mathrm{N}^{3} \mathrm{MHV}$ and $\overline{\mathrm{N}^{3} \mathrm{MHV}}$ amplitudes (i.e., for any amplitude with up to 10 external states). As in momentum space, our formalism obscures the underlying cyclic symmetry of the amplitudes, though this is encoded in the algebraic relations we have written down. Obtaining and making sense of the formulae for arbitrary tree amplitudes is likely to require a better command of these symmetries than we currently possess.

\subsection{Some elementary loop amplitudes}

The structure of loop amplitudes in twistor space has already been much discussed in [15, $16,18,20])$. As with the twistor structure of tree amplitudes, these articles typically only identified the support of the amplitudes, rather than giving explicit twistor formulae. There is no problem in principle in obtaining twistor loop amplitudes by taking the half Fourier transform of the known momentum space expressions. More interesting would be to translate the generalised unitarity methods - currently the definitive way of constructing supersymmetric loop amplitudes. In this subsection, we content ourselves with calculating the half Fourier transform explicitly for the simplest case of the 4-particle, 1 loop amplitude. Via the BDS ansätz [7] (verified up to three loops and consistent with both the dual conformal anomaly equation of Drummond et al. [60] and the strong-coupling limit computed by Alday \& Maldacena in [61]), this one loop amplitude forms the basis of the 4-particle amplitude to all orders. At the end of the subsection, we make some simple comments about some non-supersymmetric 1 loop amplitudes, in particular calculating the non-supersymmetric all-plus 1 loop amplitude.

In momentum space, the 4-particle, 1 loop amplitude is a multiple of the tree amplitude given by $[70,71]$

$$
A^{1 \text { loop }}(1,2,3,4)=\left[-\frac{1}{\epsilon^{2}}\left(\frac{\mu_{\mathrm{IR}}^{2}}{-s}\right)^{\epsilon}+\frac{1}{\epsilon^{2}}\left(\frac{\mu_{\mathrm{IR}}^{2}}{-t}\right)^{\epsilon}+\frac{1}{2} \ln ^{2} \frac{s}{t}\right] A^{\text {tree }}(1,2,3,4)+\mathcal{O}(\epsilon)
$$


at some renormalisation scale $\mu_{\mathrm{IR}}$, and where $s=\left(p_{1}+p_{2}\right)^{2}$ and $t=\left(p_{2}+p_{3}\right)^{2}$. This naturally divides into the infrared divergent part (the first two terms in the square bracket) and a finite part (the $3^{\text {rd }}$ term in the square bracket). On the support of the 4-particle momentum $\delta$-function, $s / t$ can be expressed entirely in terms of the unprimed spinors:

$$
\frac{s}{t}=\frac{\langle 12\rangle\langle 34\rangle}{\langle 14\rangle\langle 23\rangle} .
$$

Since these unprimed spinors do not participate in the half Fourier transform, the finite part of the twistor amplitude is simply

$$
\mathcal{A}_{\text {finite }}^{1 \text { loop }}(1,2,3,4)=\frac{1}{2} \ln ^{2}\left(\frac{\langle 12\rangle\langle 34\rangle}{\langle 14\rangle\langle 23\rangle}\right) \mathcal{A}^{\text {tree }}(1,2,3,4) .
$$

To study the superconformal properties of this amplitude, recall that the tree level amplitude can be written as

$$
\mathcal{A}^{\text {tree }}(1,2,3,4)=\int \frac{\mathrm{d} a}{a} \frac{\mathrm{d} b}{b} \frac{\mathrm{d} c}{c} \frac{\mathrm{d} g}{g} \delta^{(4 \mid 4)}\left(\mathrm{W}_{2}-a \mathrm{~W}_{3}-b \mathrm{~W}_{1}\right) \delta^{(4 \mid 4)}\left(\mathrm{W}_{4}-c \mathrm{~W}_{1}-g \mathrm{~W}_{3}\right) .
$$

On the support of the delta functions in (5.35) one has

$$
\frac{\langle 12\rangle\langle 34\rangle}{\langle 14\rangle\langle 23\rangle}=-\frac{a c}{b g}
$$

by using the $\lambda_{A}$ components of each of the delta functions to perform the integrals. Hence

$$
\begin{aligned}
\mathcal{A}_{\text {finite }}^{1 \text { loop }}(1,2,3,4)=\int \frac{\mathrm{d} a}{a} \frac{\mathrm{d} b}{b} & \frac{\mathrm{d} c}{c} \frac{\mathrm{d} g}{g} \frac{1}{2} \ln ^{2}\left(-\frac{a c}{b g}\right) \\
& \times \delta^{(4 \mid 4)}\left(\mathrm{W}_{2}-a \mathrm{~W}_{3}-b \mathrm{~W}_{1}\right) \delta^{(4 \mid 4)}\left(\mathrm{W}_{4}-c \mathrm{~W}_{1}-g \mathrm{~W}_{3}\right)
\end{aligned}
$$

which makes manifest the superconformal invariance of the IR-finite part of the 1 loop amplitude.

The transform of the IR divergent part is more delicate. A distinction between our split signature context, and Lorentz signature is that $s$ and $t$ can change sign and there is some ambiguity as to how the functions $(-s)^{\epsilon}$ and $(-t)^{\epsilon}$ should be continued across the zero set of $s$ and $t$. Once such a choice is made, the integral for the half Fourier transform can be reduced to a standard known one as follows: Consider the half Fourier transform of $(-s)^{-e p s i l o n} A^{\text {tree }}$ (the second term is identical). We can formally replace $(-s)^{-\epsilon}$ by the pseudo-differential operator $\left(\langle 12\rangle\left[\partial_{1} \partial_{2}\right]\right)^{-\epsilon}$, which then acts on the tree amplitude

$$
\langle 12\rangle^{-\epsilon}\left[\partial_{1} \partial_{2}\right]^{-\epsilon} \mathcal{A}^{\text {tree }}(1,2,3,4) .
$$

From (C.21), the tree amplitude $\mathcal{A}^{\text {tree }}(1,2,3,4)$ can be expressed in the Poincaré invariant form

$$
\mathcal{A}^{\text {tree }}=\frac{\langle 34\rangle^{2}}{\langle 12\rangle\langle 23\rangle\langle 34\rangle\langle 41\rangle} \delta^{(2 \mid 4)}\left(\mu_{1}+\mu_{3} \frac{\langle 41\rangle}{\langle 34\rangle}+\mu_{4} \frac{\langle 13\rangle}{\langle 34\rangle}\right) \delta^{(2 \mid 4)}\left(\mu_{2}+\mu_{3} \frac{\langle 42\rangle}{\langle 34\rangle}+\mu_{4} \frac{\langle 23\rangle}{\langle 34\rangle}\right)
$$


The action of $\left(\langle 12\rangle\left[\partial_{1} \partial_{2}\right]\right)^{-\epsilon}$ on these delta functions can be understood by translation of its action on $\delta^{(2)}\left(\mu_{1}\right) \delta^{(2)}\left(\mu_{2}\right)$, which in turn can be understood from its Fourier transform

$$
\left[\partial_{1} \partial_{2}\right]^{-\epsilon} \delta^{(2)}\left(\mu_{1}\right) \delta^{(2)}\left(\mu_{2}\right)=\int \mathrm{d}^{2} \tilde{\lambda}_{1} \mathrm{~d}^{2} \tilde{\lambda}_{2}\left[\tilde{\lambda}_{1} \tilde{\lambda}_{2}\right]^{-\epsilon} \mathrm{e}^{\mathrm{i}\left[\mu_{1} \tilde{\lambda}_{1}\right]+\mathrm{i}\left[\mu_{2} \tilde{\lambda}_{2}\right]}
$$

By thinking the pair $q=\left(\tilde{\lambda}_{1}, \tilde{\lambda}_{2}\right)$ as a 4 -vector, dual to the 4 -vector $y=\left(\mu_{1}, \mu_{2}\right)$, this can be reduced to a standard form

$$
\int \mathrm{d}^{4} q(q \cdot q)^{-\epsilon} \mathrm{e}^{\mathrm{i} q \cdot y}
$$

but where the quadratic form $q \cdot q$ has signature ++-- . For various choices of quadratic form $q \cdot q$, the Fourier transform (5.41) can be found in the tables at the end of [69]. For example, if for $(q \cdot q)^{-\epsilon}$ we take $(q \cdot q+i 0)^{-\epsilon}$ (the analytic continuation through the upper-half plane) we obtain

$$
-\frac{2^{4-2 \epsilon} \pi^{2} \Gamma(2-\epsilon)}{\Gamma(\epsilon)}(y \cdot y-i 0)^{-2+\epsilon}
$$

where in our context $(y \cdot y-i 0)^{-2+\epsilon}=\left(\left[\mu_{1} \mu_{2}\right]-i 0\right)^{-2+\epsilon}$ is understood to be analytically continued through the lower half plane. Putting this together with the translation, we obtain the final form

$$
\begin{aligned}
- & \frac{\mu_{\mathrm{IR}}^{2 \epsilon}}{\epsilon^{2}} \frac{2^{4-2 \epsilon} \pi^{2} \epsilon \Gamma(2-\epsilon)}{\Gamma(1+\epsilon)}\langle 12\rangle^{-\epsilon}\left(\left(\mu_{1}+\mu_{3} \frac{\langle 41\rangle}{\langle 34\rangle}+\mu_{4} \frac{\langle 13\rangle}{\langle 34\rangle}\right) \cdot\left(\mu_{2}+\mu_{3} \frac{\langle 42\rangle}{\langle 34\rangle}+\mu_{4} \frac{\langle 23\rangle}{\langle 34\rangle}\right)\right)^{-2+\epsilon} \\
& \times \frac{\langle 34\rangle^{2}}{\langle 12\rangle\langle 23\rangle\langle 34\rangle\langle 41\rangle} \delta^{(0 \mid 4)}\left(\chi_{1}+\chi_{3} \frac{\langle 41\rangle}{\langle 34\rangle}+\chi_{4} \frac{\langle 41\rangle}{\langle 34\rangle}\right) \delta^{(0 \mid 4)}\left(\chi_{2}+\chi_{3} \frac{\langle 42\rangle}{\langle 34\rangle}+\chi_{4} \frac{\langle 23\rangle}{\langle 34\rangle}\right)
\end{aligned}
$$

for IR divergence in the $s$-channel. The $t$-channel divergence follows by cyclic permutation.

We finish with the rather more straightforward example the all + helicity amplitude. This amplitude vanishes in the supersymmetric theory, but in the non-supersymmetric case it is non-zero at one loop, and given by the rational expression

$$
A_{+}^{1 \text { loop }}(1, \ldots, n)=\frac{-\mathrm{i}}{48 \pi^{2}} \delta^{(4)}\left(\sum p_{i}\right) \sum_{1 \leq i_{1}<i_{2}<i_{3}<i_{4} \leq n} \frac{\left\langle i_{1} i_{2}\right\rangle\left\langle i_{3} i_{4}\right\rangle\left[i_{2} i_{3}\right]\left[i_{4} i_{1}\right]}{\langle 12\rangle\langle 23\rangle \ldots\langle n 1\rangle}
$$

This is easily transformed to give

$$
\mathcal{A}_{+}^{1 \text { loop }}(1, \ldots, n)=\frac{-\mathrm{i}}{48 \pi^{2}} \sum_{1 \leq i_{1}<i_{2}<i_{3}<i_{4} \leq n}\left\langle i_{1} i_{2}\right\rangle\left\langle i_{3} i_{4}\right\rangle\left[\partial_{i_{2}} \partial_{i_{3}}\right]\left[\partial_{i_{4}} \partial_{i_{1}}\right] \mathcal{A}_{\mathrm{MHV}}^{\mathcal{N}=0}(1,2, \ldots, n)
$$

where by $\mathcal{A}_{\mathrm{MHV}}^{\mathcal{N}=0}(1,2, \ldots, n)$ we mean the formula obtained from (3.29) by replacing $\delta^{(2 \mid 4)}$ or $\tilde{\delta}^{(2 \mid 4)}$ by $\delta^{(2 \mid 0)}$ or $\tilde{\delta}^{(2 \mid 0)}$, respectively. The formula therefore has derivative of delta function support along a line as predicted in [15]. 


\section{Twistor supergravity amplitudes}

In this section, we sketch how the recursion rule works for $\mathcal{N}=8$ supergravity as far as the homogeneous term and its conjugate, and find formulae for the MHV and $\overline{\mathrm{MHV}}$ amplitudes. The BCFW relation itself is the same:

$$
\begin{aligned}
\mathcal{M}(1, \ldots, n)=\sum \int & D^{3 \mid 8} \mathrm{~W} \mathcal{M}_{L}(1, \ldots, \mathrm{W}) \\
& \times \operatorname{sgn}\left(\left\langle\mathrm{W}_{1} \mathrm{~W}\right\rangle \mathrm{W}_{1} \cdot \frac{\partial}{\partial \mathrm{W}_{n}}\left[\frac{\partial}{\partial \mathrm{W}} \frac{\partial}{\partial \mathrm{W}_{n}}\right]\right) \mathcal{M}_{R}\left(\mathrm{~W}, \ldots, \mathrm{W}_{n}\right),
\end{aligned}
$$

except that the sum now runs over all ways of partitioning the external legs over the two sub-amplitudes, with no cyclicity requirement.

To compute the three-point seed amplitudes we start with the momentum space formulae

$$
\begin{aligned}
M_{\mathrm{MHV}}(1,2,3) & =\frac{\delta^{(4 \mid 16)}\left(\sum_{i=1}^{3}|i\rangle \llbracket 1 \|\right)}{\langle 12\rangle^{2}\langle 23\rangle^{2}\langle 31\rangle^{2}} \\
M_{\overline{\mathrm{MHV}}}(1,2,3) & =\frac{\delta^{(4)}\left(p_{1}+p_{2}+p_{3}\right) \delta^{(8)}\left(\eta_{1}[23]+\eta_{2}[31]+\eta_{3}[12]\right)}{[12]^{2}[23]^{2}[31]^{2}}
\end{aligned}
$$

which are simply the squares of the Yang-Mills three-point amplitudes [62], provided one strips away the momentum conserving delta functions. We will see a somewhat different structure in twistor space, though the gravitational and Yang-Mills seed amplitudes are still closely related.

Taking the half Fourier transform, the (dual) twistor form of the 3-point MHV amplitude is

$$
\mathcal{M}_{\mathrm{MHV}}\left(\mathrm{W}_{1}, \mathrm{~W}_{2}, \mathrm{~W}_{3}\right)=\frac{\delta^{(2 \mid 8)}\left(\mu_{1}\langle 23\rangle+\mu_{2}\langle 31\rangle+\mu_{3}\langle 12\rangle\right)}{\langle 12\rangle^{2}\langle 23\rangle^{2}\langle 31\rangle^{2}} .
$$

This amplitude has homogeneity +2 in each of its arguments as required for on-shell $\mathcal{N}=8$ supermultiplets. We can write (6.3) as

$$
\begin{aligned}
\mathcal{M}_{\mathrm{MHV}}\left(\mathrm{W}_{1}, \mathrm{~W}_{2}, \mathrm{~W}_{3}\right) & =\left|\left\langle\mathrm{W}_{2} \mathrm{~W}_{3}\right\rangle \mathrm{iW}_{2} \cdot \frac{\partial}{\partial \mathrm{W}_{1}} \mathrm{iW}_{3} \cdot \frac{\partial}{\partial \mathrm{W}_{1}}\right| \delta^{(4 \mid 8)}\left(\mathrm{W}_{1}\right) \\
& =\left(\left\langle\mathrm{W}_{2} \mathrm{~W}_{3}\right\rangle \mathrm{iW}_{2} \cdot \frac{\partial}{\partial \mathrm{W}_{1}} \mathrm{iW}_{3} \cdot \frac{\partial}{\partial \mathrm{W}_{1}}\right) \mathcal{H}_{23}^{1} \delta^{(4 \mid 8)}\left(\mathrm{W}_{1}\right)
\end{aligned}
$$

in close analogy to the form (3.34) of the SYM amplitude in terms of the $\mathcal{H}$-operator; in this formula the sgn-factor in $\mathcal{H}$ turns the ordinary differential operator into its formal modulus. Equivalently, in terms of the $\mathcal{N}=8$ collinear delta function of equation (3.12), this is

$$
\mathcal{M}_{\mathrm{MHV}}\left(\mathrm{W}_{1}, \mathrm{~W}_{2}, \mathrm{~W}_{3}\right)=\left|\left\langle\mathrm{W}_{2} \mathrm{~W}_{3}\right\rangle\right| \tilde{\delta}^{(2 \mid 8)}\left(\mathrm{W}_{1} ; \mathrm{W}_{2}, \mathrm{~W}_{3}\right)
$$

The explicit factor of $\left|\left\langle\mathrm{W}_{2} \mathrm{~W}_{3}\right\rangle\right|$ here breaks $\mathrm{SL}(4 \mid 8, \mathbb{R})$ invariance, but $\mathcal{N}=8$ superPoincaré invariance is preserved. Although (6.4) appears to single out state 1, it is clear from (6.3) that the amplitude is really symmetric under exchange of any two states. 
The 3-point $\overline{\mathrm{MHV}}$ supergravity amplitude in twistor space is

$$
\begin{aligned}
\mathcal{M}_{\overline{\mathrm{MHV}}}\left(\mathrm{W}_{1}, \mathrm{~W}_{2}, \mathrm{~W}_{3}\right) & =\left|\left[\frac{\partial}{\partial \mathrm{W}_{2}} \frac{\partial}{\partial \mathrm{W}_{3}}\right] \mathrm{iW}_{1} \cdot \frac{\partial}{\partial \mathrm{W}_{2}} \mathrm{iW}_{1} \cdot \frac{\partial}{\partial \mathrm{W}_{3}}\right| \delta_{1}^{(4 \mid 8)}\left(\mathrm{W}_{2}\right) \delta_{1}^{(4 \mid 8)}\left(\mathrm{W}_{3}\right) \\
& =\left(\left[\frac{\partial}{\partial \mathrm{W}_{2}} \frac{\partial}{\partial \mathrm{W}_{3}}\right] \mathrm{iW}_{1} \cdot \frac{\partial}{\partial \mathrm{W}_{2}} \mathrm{iW}_{1} \cdot \frac{\partial}{\partial \mathrm{W}_{3}}\right) \widetilde{\mathcal{H}}_{1}^{23} \delta^{(4 \mid 8)}\left(\mathrm{W}_{2}\right) \delta^{(4 \mid 8)}\left(\mathrm{W}_{3}\right)
\end{aligned}
$$

in close analogy to (3.32) for SYM (again we have used $\tilde{\mathcal{H}}$ to formally turn a differential operator into its modulus). Once again, this may be written in terms of weighted projective delta functions as

$$
\mathcal{M}_{\overline{\mathrm{MHV}}}\left(\mathrm{W}_{1}, \mathrm{~W}_{2}, \mathrm{~W}_{3}\right)=\left|\left[\frac{\partial}{\partial \mathrm{W}_{2}} \frac{\partial}{\partial \mathrm{W}_{3}}\right]\right| \tilde{\delta}^{(3 \mid 8)}\left(\mathrm{W}_{2}, \mathrm{~W}_{1}\right) \tilde{\delta}^{(3 \mid 8)}\left(\mathrm{W}_{3}, \mathrm{~W}_{1}\right) .
$$

\subsection{The homogeneous term and the MHV amplitudes}

The homogeneous term for an $\mathrm{N}^{k} \mathrm{MHV}$ amplitude again takes the form

$$
\sum_{r \neq 1, n} \int D^{3 \mid 8} \mathrm{~W} \mathcal{M}_{k}(1, \ldots, \mathrm{W}) \operatorname{sgn}\left(\langle 1 \mathrm{~W}\rangle \mathrm{W}_{1} \cdot \partial_{\mathrm{W}}\left[\partial_{\mathrm{W}} \partial_{n}\right]\right) \mathcal{M}_{-1}(\mathrm{~W}, r, n)
$$

in which the sum over partitions has reduced to a sum over the external state $r$ attached to the three-point $\overline{\mathrm{MHV}}$ sub-amplitude on the right (and hence absent from $\mathcal{M}_{k}(1, \ldots, \mathrm{W})$ ). Recalling that

$$
\mathcal{M}_{-1}(\mathrm{~W}, r, n)=\left|\left[\partial_{\mathrm{W}} \partial_{n}\right] \mathrm{W}_{r} \cdot \partial_{\mathrm{W}} \mathrm{W}_{r} \cdot \partial_{n}\right| \delta^{(4 \mid 8)}(\mathrm{W}) \delta^{(4 \mid 8)}\left(\mathrm{W}_{n}\right),
$$

the factor $\operatorname{sgn}\left[\partial_{\mathrm{W}} \partial_{n}\right]$ in the recursion relation combines with $\left|\left[\partial_{\mathrm{W}} \partial_{n}\right]\right|$ in the amplitude to form the standard differential operator $\left[\partial_{\mathrm{W}} \partial_{n}\right]$. We then integrate by parts so that $\partial_{\mathrm{W}}$ acts on $\mathcal{M}_{k}(1, \ldots, W)$. Since

$$
\left|\mathrm{W}_{r} \cdot \frac{\partial}{\partial \mathrm{W}}\right| \delta^{(4 \mid 8)}(\mathrm{W})=\int \frac{\mathrm{d} t}{t^{2}} \delta^{(4 \mid 8)}\left(\mathrm{W}-t \mathrm{~W}_{r}\right)=\tilde{\delta}^{(3 \mid 8)}\left(\mathrm{W}, \mathrm{W}_{r}\right)
$$

the integral is straightforward and leaves us with

$$
\begin{gathered}
-\sum_{r \neq 1, n} \frac{\partial}{\partial \mu_{r}^{A^{\prime}}}\left(\mathcal{M}_{k}(1, \ldots, r)\right) \frac{\partial}{\partial \mu_{n A^{\prime}}}\left\{\operatorname{sgn}\left(\langle 1 r\rangle \mathrm{W}_{1} \cdot \frac{\partial}{\partial \mathrm{W}_{n}}\right)\left|\mathrm{W}_{r} \cdot \frac{\partial}{\partial \mathrm{W}_{n}}\right| \delta^{(4 \mid 8)}\left(\mathrm{W}_{n}\right)\right\} \\
=-\sum_{r \neq 1, n} \frac{\partial}{\partial \mu_{r}^{A^{\prime}}}\left(\mathcal{M}_{k}(1, \ldots, r)\right) \frac{\partial}{\partial \mu_{n A^{\prime}}}\left\{\mathrm{W}_{r} \cdot \frac{\partial}{\partial \mathrm{W}_{n}} \mathcal{H}_{1 r}^{n} \delta^{(4 \mid 8)}\left(\mathrm{W}_{n}\right)\right\}
\end{gathered}
$$

In the second line, we have written $\left|\mathrm{W}_{1} \cdot \partial_{n}\right|=\mathrm{W}_{1} \cdot \partial_{n} \operatorname{sgn}\left(\mathrm{W}_{1} \cdot \partial_{n}\right)$ to pull out the operator $\mathcal{H}_{r 1}^{n}$. If we introduce the operator

$$
\mathcal{G}_{23}^{1}:=\left|\mathrm{W}_{2} \cdot \partial_{1}\right| \operatorname{sgn}\left(\langle 23\rangle \mathrm{W}_{3} \cdot \partial_{1}\right)=\mathrm{W}_{2} \cdot \partial_{1} \mathcal{H}_{32}^{1},
$$

then the homogeneous term can be written more compactly as

$$
-\sum_{r \neq 1, n} \mathcal{G}_{r 1}^{n}\left[\partial_{r} \partial_{n}\right] \delta^{(4 \mid 8)}\left(\mathrm{W}_{n}\right) \mathcal{M}_{k}(1, \ldots, r) .
$$


For MHV amplitudes, the homogeneous term is the complete recursion, and iteration of (6.13) gives

$$
\mathcal{M}_{\mathrm{MHV}}(1, \ldots, n)=\sum_{\mathcal{P}(2, \ldots, n-1)}\left(\prod_{r=4}^{n} \mathcal{G}_{r-1,1}^{r}\left[\partial_{r}, \partial_{r-1}\right] \delta^{(4 \mid 8)}\left(\mathrm{W}_{r}\right)\right) \mathcal{M}_{\mathrm{MHV}}(1,2,3)
$$

where $\mathcal{P}(2, \ldots, n-1)$ denotes the permutations of the labels 2 to $n$ and, because the terms in the product do not commute, they are ordered to the left in increasing $r$.

\subsection{The conjugate homogeneous term and $\overline{\mathrm{MHV}}$ amplitudes}

The conjugate homogeneous term in the decomposition of an $\mathrm{N}^{k+1} \mathrm{MHV}$ amplitude is

$$
\sum_{r \neq 1, n} \int D^{3 \mid 8} \mathrm{~W} \mathcal{M}_{0}(1, r, \mathrm{~W}) \operatorname{sgn}\left(\langle 1 \mathrm{~W}\rangle \mathrm{W}_{1} \cdot \partial_{n}\left[\partial_{\mathrm{W}} \partial_{n}\right]\right) \mathcal{M}_{k}(\mathrm{~W}, \ldots, n)
$$

where

$$
\begin{aligned}
\mathcal{M}_{0}(1, r, \mathrm{~W}) & =\left|\langle 1 \mathrm{~W}\rangle \mathrm{W} \cdot \frac{\partial}{\partial \mathrm{W}_{r}} \mathrm{~W}_{1} \cdot \frac{\partial}{\partial \mathrm{W}_{r}}\right| \delta^{(4 \mid 8)}\left(\mathrm{W}_{r}\right) \\
& =\left|\langle 1 \mathrm{~W}\rangle \mathrm{W}_{1} \cdot \frac{\partial}{\partial \mathrm{W}_{r}}\right| \tilde{\delta}^{(3 \mid 8)}\left(\mathrm{W}_{r}, \mathrm{~W}\right) .
\end{aligned}
$$

This $\delta$-function again allows us to perform the integral, setting $\mathrm{W}=\mathrm{W}_{r}$. We are left with the contribution

$$
\sum_{r \neq 1, n}\langle 1 r\rangle \mathrm{W}_{1} \cdot \frac{\partial}{\partial \mathrm{W}_{r}} \widetilde{\mathcal{H}}_{1}^{r n} \mathcal{M}_{k}(r, \ldots, n) .
$$

Again, defining

$$
\tilde{\mathcal{G}}_{3}^{12}=\left|\mathrm{W}_{3} \cdot \partial_{2}\right| \operatorname{sgn}\left(\left[\partial_{1} \partial_{2}\right] \mathrm{W}_{3} \cdot \partial_{1}\right)=\mathrm{W}_{3} \cdot \partial_{2} \tilde{\mathcal{H}}_{3}^{12}
$$

we can write

$$
\sum_{r \neq 1, n}\langle 1 r\rangle \widetilde{\mathcal{G}}_{1}^{n r} \mathcal{M}_{k}(r, \ldots, n),
$$

and this can be understood as inserting the particle 1. In particular, this is the complete $\mathrm{BCFW}$ decomposition for an $\overline{\mathrm{MHV}}$ amplitude, so we recursively obtain

$$
\mathcal{M}_{n-4}(1, \ldots, n)=\sum_{\mathcal{P}(2, \ldots, n-1)}\left(\prod_{r=2}^{n-2}\langle r r+1\rangle \widetilde{\mathcal{G}}_{r+1}^{n r}\right) \mathcal{M}_{-1}(1,2, n),
$$

where again the ordering is important in the product which is ordered to the left with increasing $r$.

\section{An ambidextrous approach}

For the most part in this paper we have focussed on transforming amplitudes and their recursion relations from momentum space to dual twistor space. We could equally have chosen to transform some particles to twistor space and others to dual twistor space. $A$ priori, there is no clear rule as to which external particles should be expressed in terms 
of twistors and which in terms of dual twistors ${ }^{20}$ but a choice was recently discovered by Arkani-Hamed, Cachazo, Cheung \& Kaplan [46] that leads to significant simplifications for the BCFW recursion. In this section we will first discuss the 'twistor transform' that moves from twistor space to dual twistor space, and then use this transform to relate their formulae directly to ours.

\subsection{Fourier transforms and twistor transforms}

Thus far we have taken the half Fourier transform from functions $\Phi(\lambda, \tilde{\lambda})$ on the split signature light cone in momentum space to functions on real dual twistor space by Fourier transforming in the $\tilde{\lambda}_{A^{\prime}}$ variable to obtain

$$
f(W):=f(\lambda, \mu)=\int \mathrm{d}^{2} \tilde{\lambda} \mathrm{e}^{\mathrm{i}[\mu \tilde{\lambda}]} \Phi(\lambda, \tilde{\lambda})
$$

as in equation (B.13). We could just as easily have Fourier transformed in $\lambda_{A}$ to obtain a function on twistor space

$$
F(Z)=F(\omega, \pi)=\int \mathrm{d}^{2} \lambda \mathrm{e}^{-\mathrm{i}\langle\omega \lambda\rangle} \Phi(\lambda, \pi)
$$

with co-ordinates $Z^{\alpha}=\left(\omega^{A}, \pi_{A^{\prime}}\right)$ on twistor space (after relabelling $\left.\tilde{\lambda}_{A^{\prime}}=\pi_{A^{\prime}}\right)$. Combining these two half Fourier transforms, one obtains a map from functions on twistor space to functions on dual twistor space, given by

$$
f(W)=\frac{1}{(2 \pi)^{2}} \int \mathrm{d}^{4} Z \mathrm{e}^{-\mathrm{i} Z \cdot W} F(Z),
$$

where $Z \cdot W=\omega^{A} \lambda_{A}+\pi_{A^{\prime}} \mu^{A^{\prime}}$.

Although (7.3) is clearly implied by the combined half Fourier transforms, it has some rather puzzling features. The functions $f(W)$ and $F(Z)$ are homogeneous functions on (dual) twistor space, with some well-defined weights $n$ and $-n-4$ respectively. However, if $F(Z)$ has negative weight, then (7.3) diverges at the origin, while if it has positive weight then (7.3) diverges at infinity. So the transform appears not to make sense.

To resolve this issue, we must understand equation (7.3) as a Fourier transform of distributions. To make this explicit and to obtain projective formulae, we co-ordinatise $\mathbb{R}^{4}$ by $Z=r \Theta$ where $r \in(-\infty, \infty)$ and $\Theta=\Theta\left(\theta_{i}\right)$ lies on a hemisphere of unit radius on which the $\theta_{i}$ are co-ordinates. ${ }^{21}$ The $\theta_{i}$ also provide co-ordinates on $\mathbb{R P}^{3}$. We will not need to make the co-ordinatisation of $\mathbb{R} \mathbb{P}^{3}$ explicit and just denote its volume form by $\mathrm{D}^{3} \Theta$. This is defined so that

$$
\mathrm{d}^{4} Z=|r|^{3} \mathrm{~d} r \wedge \mathrm{D}^{3} \Theta,
$$

\footnotetext{
${ }^{20}$ If one wishes to describe $\mathcal{N}=4 \mathrm{SYM}$ using only manifest $\mathcal{N}=3$ supersymmetry (or explicit $\mathcal{N}=7$ supersymmetry for $\mathcal{N}=8 \mathrm{SG}$ ), there are naturally two multiplets, one starting from the lowest helicity and one from the highest. In this case it is natural to transform one multiplet to twistor space and the other on dual twistor space. This approach fits in naturally both with twistor diagrams and with the ambitwistor action [64] at the expense of either losing the economy of dealing with the whole spectrum in a single supermultiplet, or having a redundant representation.

${ }^{21}$ These are not quite the usual polar co-ordinates, because $r$ lies on a complete line, not a half line, and correspondingly, $\theta_{i}$ lives on a hemisphere or $\mathbb{R P}^{3}$ rather than a complete sphere.
} 
where the modulus sign arises from the Jacobian of the co-ordinate transformation. Such a choice is not projectively invariant, and two such choices will differ by some scaling $(r, \Theta) \rightarrow\left(r^{\prime}, \Theta^{\prime}\right)=\left(a r, a^{-1} \Theta\right)$ where $a=a(\Theta)$.

The $r$ dependence in (7.3) can now be made explicit. Assuming $F(Z)$ has homogeneity $-n-4$, one finds

$$
f(W)=\frac{1}{(2 \pi)^{2}} \int \operatorname{sgn}(r) r^{-n-1} \mathrm{e}^{\mathrm{i} r \Theta \cdot W} F(\Theta) \mathrm{d} r \mathrm{D}^{3} \Theta .
$$

The integral for $r$ is a Fourier transform with conjugate variable $\Theta \cdot W$. For $n \leq 0$ this integral blows up as $r \rightarrow \infty$, and for $n \geq 0$ it blows up at the origin. These integrals all have a standard regularisation (see e.g. [69] for a detailed exposition): For $n<0$ one obtains

$$
\int r^{-n-1} \operatorname{sgn}(r) \mathrm{e}^{\mathrm{i} r s} \mathrm{~d} r=2 s^{n} \mathrm{i}^{-n}(-n-1) ! .
$$

and for $n \geq 0$

$$
\int r^{-n-1} \operatorname{sgn}(r) \mathrm{e}^{\mathrm{i} r s} \mathrm{~d} r=2 \frac{\mathrm{i}^{n+1}}{n !} s^{n}\left(\log |s|-\alpha_{n}\right)
$$

where $\alpha_{n}=\Gamma^{\prime}(1)+\sum_{k=1}^{n} 1 / k$. Note that $\Gamma^{\prime}(1)=-\gamma$, where $\gamma$ is Euler's constant. ${ }^{22}$ Thus, having integrated out the scale of $Z$, we obtain the projective formulae

$$
f(W)= \begin{cases}\frac{2 \mathrm{i}^{-n}(-n-1) !}{(2 \pi)^{2}} \int D^{3} Z F(Z)(Z \cdot W)^{n} & n \leq-1 \\ \frac{2 \mathrm{i}^{n+1}}{(2 \pi)^{2} n !} \int D^{3} Z F(Z)(Z \cdot W)^{n}\left(\log |Z \cdot W|-\alpha_{n}\right) & n \geq 0\end{cases}
$$

where $D^{3} Z=\varepsilon_{\alpha \beta \gamma \delta} Z^{\alpha} \mathrm{d} Z^{\beta} \wedge \mathrm{d} Z^{\gamma} \wedge \mathrm{d} Z^{\delta}$ is the canonical top degree form of weight +4 on projective twistor space.

To check the projective invariance we re-scale $Z \rightarrow a(Z) Z$. Invariance is obvious when $n<0$, but for $n \geq 0$, the rhs changes by an arbitrary polynomial of degree $n$ in $W$. While this may seem to violate projective invariance, in fact it is natural to think of a dual twistor function $f(W)$ of homogeneity $n>0$ as being defined modulo polynomials of degree $n$. This is because the $\mathrm{X}$-ray (or Penrose) transform

$$
\phi_{A_{1} \ldots A_{n+2}}(x)=\int\langle\lambda \mathrm{d} \lambda\rangle \frac{\partial^{n+2}}{\partial \lambda_{A_{1}} \cdots \partial \lambda_{A_{n+2}}} f\left(\lambda_{B}, x^{C C^{\prime}} \lambda_{C}\right)
$$

gives a vanishing space-time field when $f(W)$ is a degree $n$ polynomial. Thus the constant $\alpha_{n}$ only contributes such a polynomial to $f(W)$, and is thus irrelevant here. With this proviso, equation (7.8) now respects the homogeneities and is a clear analogue of the complex version of the twistor transform [39].

We must also consider twistor and dual twistor functions $\tilde{F}(Z)$ and $\tilde{f}(W)$ with 'wrong sign' behaviour as in (3.7). In this case, integrating out the scale yields the Fourier transform of $r^{-n-1}$ without the sgn $r$ factor:

$$
\int \mathrm{d} r \mathrm{e}^{\mathrm{i} r s} r^{-n-1}= \begin{cases}\frac{\mathrm{i}^{n+1} \pi s^{n}}{n !} \operatorname{sgn}(s) & n \geq 0 \\ 2 \pi(-\mathrm{i})^{-n-1} \delta^{-n-1}(s) & n \leq-1,\end{cases}
$$

\footnotetext{
${ }^{22}$ This gives an alternative derivation of Penrose's 'universal bracket factor' [72].
} 
where $\delta^{-n-1}(s)$ denotes the $(|n|-1)^{\text {th }}$ derivative of $\delta(s)$. The projective version of the twistor transform for wrong sign functions is thus

$$
\tilde{f}(W)= \begin{cases}\frac{\mathrm{i}^{n+1} \pi}{(2 \pi)^{2} n !} \int D^{3} Z \tilde{F}(Z)(Z \cdot W)^{n} \operatorname{sgn}(Z \cdot W) & n \geq 0 \\ \frac{\mathrm{i}^{n+1}}{2 \pi} \int D^{3} Z \tilde{F}(Z) \delta^{-n-1}(Z \cdot W) & n \leq-1 .\end{cases}
$$

For $n \leq-1$ in this odd-odd case, the twistor transform becomes a radon-like transform over planes in twistor space. This is possible despite the non-orientability of the planes $\left(\mathbb{R P}^{2} \mathrm{~s}\right)$ because the wrong sign behaviour is what is required to define a density on an even-dimensional projective space.

\subsubsection{Supersymmetric twistor transforms}

The positive and negative $n$ formulæ combine to form supersymmetric twistor transforms. With $\mathcal{N}$ supersymmetries, the supertwistor transform is

$$
f(\mathrm{~W})=\int D^{3 \mid \mathcal{N}} \mathrm{Z} F(\mathrm{Z})(\mathrm{Z} \cdot W)^{\mathcal{N} / 2-2} \log |\mathrm{Z} \cdot \mathrm{W}|
$$

where $F(\mathrm{Z})$ and $f(\mathrm{~W})$ each have homogeneity $\mathcal{N} / 2-2$ and are of 'right sign' type. The 'wrong sign' version is

$$
\tilde{f}(\mathrm{~W})=\int D^{3 \mid \mathcal{N}} \mathrm{Z} \tilde{F}(\mathrm{Z})(\mathrm{Z} \cdot \mathrm{W})^{\mathcal{N} / 2-2} \operatorname{sgn}(\mathrm{Z} \cdot \mathrm{W}) .
$$

Expanding these transforms in powers of the Grassmann co-ordinates reproduces the transforms (7.8) \& (7.11).

\subsection{The inner product}

In Lorentzian signature, the standard inner product between two massless fields of helicity $h$ is given by multiplying the momentum space wavefunction $\Phi_{2 h}(\lambda, \tilde{\lambda})$ by the complex conjugate of the wavefunction $\Psi_{2 h}(\lambda, \tilde{\lambda})$, and then integrating over the momentum light-cone:

$$
(\Psi, \Phi)=\frac{\mathrm{i}}{(2 \pi)^{4}} \int \mathrm{d}^{3} \ell \bar{\Psi} \Phi
$$

where $\mathrm{d}^{3} \ell$ is the standard invariant measure $\mathrm{d}^{3} \ell=\langle\lambda \mathrm{d} \lambda\rangle \mathrm{d}^{2} \tilde{\lambda}-[\tilde{\lambda} \mathrm{d} \tilde{\lambda}] \mathrm{d}^{2} \lambda$. This Lorentzian inner product is anti-linear in $\Psi$ because of the complex conjugation; in particular $\bar{\Psi}$ has helicity $-h$ because the conjugation exchanges $\lambda$ and $\tilde{\lambda}$.

On $\mathbb{R}^{2,2}$, complex conjugation does not change particle helicity, so instead of an inner product we simply have a bilinear pairing between fields of helicity $h$ and fields of helicity $-h$, given by

$$
\left(\Psi_{-2 h}, \Phi_{2 h}\right)=\frac{1}{(2 \pi)^{4}} \int \mathrm{d}^{3} \ell \Psi_{-2 h} \Phi_{2 h}
$$


The corresponding pairing on twistor space is between a twistor function of weight $n$ and a twistor function of weight $-n-4$. This pairing is again given by multiplying the twistor functions and integrating over the real projective twistor space:

$$
(F, G)=\int_{\mathbb{R}^{3}} \mathrm{D}^{3} Z F_{n}(Z) G_{-n-4}(Z) .
$$

Unitarity of the half Fourier transform ensures that the momentum space and twistor pairings agree. On twistor space, when $n \geq 0$ or $n \leq-4$ the fact that positive homogeneity twistor functions are defined modulo polynomials is dual to the fact that for (7.16) to be well-defined, the charge integral

$$
Q^{\alpha \beta \ldots \gamma}:=\int D^{3} Z Z^{\alpha} Z^{\beta} \cdots Z^{\gamma} F_{n}(Z)
$$

must vanish, where $F_{n}(Z)$ has homogeneity $n \leq-4$ and there are $|n|-4$ factors of $Z$ inserted in the integral. When $n=-4$ this is the standard twistor charge integral for a self-dual Maxwell field, and when $n=-6$ it yields the angular-momentum twistor of the corresponding linearised gravitational field [73, 74].

Combined with the Fourier/Twistor transform described above, we obtain a pairing between a twistor function $F(Z)$ and a dual twistor function $G(W)$ each of homogeneity degree $-n-4$. When $F(Z)$ and $G(W)$ both have the 'right sign' behaviour this pairing

$$
(F, G)= \begin{cases}\frac{2 \mathrm{i}^{-n}(-n-1) !}{(2 \pi)^{2}} \int D^{3} Z \wedge D^{3} W F(Z) G(W)(Z \cdot W)^{n} & n \leq-1 \\ \frac{2 \mathrm{i}^{n+1}}{(2 \pi)^{2} n !} \int D^{3} Z \wedge D^{3} W F(Z) G(W)(Z \cdot W)^{n} \log |Z \cdot W| & n \geq 0,\end{cases}
$$

whereas for 'wrong sign' functions $\tilde{F}(Z)$ and $\tilde{G}(W)$ it is

$$
(\tilde{F}, \tilde{G})= \begin{cases}\frac{\mathrm{i}^{n+1} \pi}{(2 \pi)^{2}} \int D^{3} Z \wedge D^{3} W \tilde{F}(Z) \tilde{G}(W) \delta^{-n-1}(Z \cdot W) & n \leq-1 \\ \frac{\mathrm{i}^{n+1} \pi}{(2 \pi)^{2} n !} \int D^{3} Z \wedge D^{3} W \tilde{F}(Z) \tilde{G}(W)(Z \cdot W)^{n} \operatorname{sgn}(Z \cdot W) & n \geq 0 .\end{cases}
$$

Once again, these formulæ combine into supersymmetric pairings given by

$$
\begin{aligned}
& (F, G)=-\frac{\mathrm{i}^{\frac{\mathcal{N}}{2}-1}}{(2 \pi)^{2}\left(\frac{\mathcal{N}}{2}-2\right) !} \int D^{3 \mid \mathcal{N}} \mathrm{Z} \wedge D^{3 \mid \mathcal{N}} \mathrm{W} F(\mathrm{Z}) G(\mathrm{~W})(\mathrm{Z} \cdot \mathrm{W})^{\frac{\mathcal{N}}{2}-2} \log |\mathrm{Z} \cdot \mathrm{W}| \\
& (\tilde{F}, \tilde{G})=\frac{\mathrm{i}^{\frac{\mathcal{N}}{2}-1} \pi}{(2 \pi)^{2}\left(\frac{\mathcal{N}}{2}-2\right) !} \int D^{3 \mid \mathcal{N}} \mathrm{Z} \wedge D^{3 \mid \mathcal{N}} \mathrm{W} \tilde{F}(\mathrm{Z}) \tilde{G}(\mathrm{~W})(\mathrm{Z} \cdot \mathrm{W})^{\frac{\mathcal{N}}{2}-2} \operatorname{sgn}(\mathrm{Z} \cdot \mathrm{W})
\end{aligned}
$$

where all the wavefunctions have homogeneity $\mathcal{N} / 2-2$.

\subsection{BCFW recursion in ambitwistor space}

We are now in position to explain the relation of the present paper to that of Arkani-Hamed et al. [46]. The main awkwardness of the twistor space BCFW formula 


$$
\begin{aligned}
\mathcal{M}\left(\mathrm{W}_{1}, \ldots, \mathrm{W}_{n}\right)=\sum & \int D^{3 \mid \mathcal{N}} \mathrm{W}_{L}\left(\mathrm{~W}_{1}, \ldots, \mathrm{W}\right) \\
& \times \operatorname{sgn}\left(\left\langle\mathrm{W}_{1} \mathrm{~W}\right\rangle \mathrm{W}_{1} \cdot \frac{\partial}{\partial \mathrm{W}_{n}}\left[\frac{\partial}{\partial \mathrm{W}} \frac{\partial}{\partial \mathrm{W}_{n}}\right]\right) \mathcal{M}_{R}\left(\mathrm{~W}, \ldots, \mathrm{W}_{n}\right)
\end{aligned}
$$

is the presence of the non-local operator $\operatorname{sgn}\left(\mathrm{W}_{1} \cdot \partial_{n}\left[\partial_{\mathrm{W}} \partial_{n}\right]\right)$. This can be rendered local by use of the Fourier/Twistor transforms introduced above. To achieve this, first represent $\mathcal{M}_{R}\left(\mathrm{~W}, \ldots, \mathrm{W}_{n}\right)$ in terms of a (non-projective) Fourier transform of $\mathcal{M}_{R}\left(\mathrm{Z}, \ldots, \mathrm{Z}_{n}\right)$ in its first and last arguments $\mathrm{Z}$ and $\mathrm{Z}_{n}$. Then

$$
\begin{aligned}
& \operatorname{sgn}\left(\mathrm{W}_{1} \cdot \frac{\partial}{\partial \mathrm{W}_{n}}\left[\frac{\partial}{\partial \mathrm{W}} \frac{\partial}{\partial \mathrm{W}_{n}}\right]\right) \mathcal{M}_{R}\left(\mathrm{~W}, \ldots, \mathrm{W}_{n}\right) \\
& \quad=\frac{1}{(2 \pi)^{4}} \int \mathrm{d}^{4 \mid \mathcal{N}} \mathrm{Zd}^{4 \mid \mathcal{N}} \mathrm{Z}_{n} \mathrm{e}^{\mathrm{i} \mathrm{Z} \cdot \mathrm{W}} \mathrm{e}^{\mathrm{i} \mathrm{Z}_{n} \cdot \mathrm{W}_{n}} \operatorname{sgn}\left(\mathrm{Z}_{n} \cdot \mathrm{W}_{1}\left[\mathrm{ZZ}_{n}\right]\right) \mathcal{M}_{R}\left(\mathrm{Z}, \ldots, \mathrm{Z}_{n}\right),
\end{aligned}
$$

where we abuse notation by not distinguishing $\mathcal{M}_{R}$ from its Fourier transform, and define $\left[\mathrm{ZZ}_{n}\right]:=\left[\pi \pi_{n}\right]=\left[\tilde{\lambda} \tilde{\lambda}_{n}\right]$. (It makes no difference whether or not we similarly transform the remaining states in $\mathcal{M}_{R}$.) The Fourier transform in $\mathrm{Z}_{n}$ may be accommodated by likewise Fourier transforming the lhs of the BCFW recursion (7.21). Since $\operatorname{sgn}\left(\left[\mathrm{ZZ}_{n}\right]\right) \mathcal{M}_{R}\left(\mathrm{Z}, \ldots, \mathrm{Z}_{n}\right)$ has wrong sign behaviour in $\mathrm{Z}$, we obtain the projective form of ambidextrous $\mathrm{BCFW}$ recursion

$$
\begin{aligned}
\mathcal{M}\left(\mathrm{W}_{1}, \ldots, \mathrm{Z}_{n}\right)=\sum \int D^{3 \mid \mathcal{N}} \mathrm{Z} & \wedge D^{3 \mid \mathcal{N}} W \mathcal{M}_{L}\left(\mathrm{~W}_{1}, \ldots, \mathrm{W}\right)(\mathrm{Z} \cdot \mathrm{W})^{\mathcal{N} / 2-2} \\
\times & \operatorname{sgn}\left(\left\langle\mathrm{W}_{1} \mathrm{~W}\right\rangle \mathrm{Z}_{n} \cdot \mathrm{W}_{1} \mathrm{Z} \cdot \mathrm{W}\left[\mathrm{ZZ}_{n}\right]\right) \mathcal{M}_{R}\left(\mathrm{Z}, \ldots, \mathrm{Z}_{n}\right)
\end{aligned}
$$

in agreement with Arkani-Hamed et. al. [46]. In this form, the non-local sign operators have been replaced by multiplication by the local operator

$$
\operatorname{sgn}\left(\left\langle\mathrm{W}_{1} \mathrm{~W}\right\rangle \mathrm{Z}_{n} \cdot \mathrm{W}_{1} \mathrm{Z} \cdot \mathrm{W}\left[\mathrm{ZZ}_{n}\right]\right)
$$

in $\mathcal{N}=4 \mathrm{SYM}$ or

$$
(\mathrm{Z} \cdot \mathrm{W})^{2} \operatorname{sgn}\left(\left\langle\mathrm{W}_{1} \mathrm{~W}\right\rangle \mathrm{Z}_{n} \cdot \mathrm{W}_{1} \mathrm{Z} \cdot \mathrm{W}\left[\mathrm{ZZ}_{n}\right]\right)
$$

in $\mathcal{N}=8 \mathrm{SG}$, at the expense of introducing a larger integral for the intermediate state. Comparing the ambitwistorial BCFW recursion (7.23) to the supersymmetric pairing $(\tilde{F}, \tilde{G})$ of $(7.20)$, we again see the close relation between BCFW recursion and the inner product of the wrong sign wavefunctions

$$
\mathcal{M}_{L}(\ldots, \mathrm{W}) \operatorname{sgn}\left(\left\langle\mathrm{W}_{1} \mathrm{~W}\right\rangle\right) \quad \text { and } \quad \mathcal{M}_{R}(\mathrm{Z}, \ldots) \operatorname{sgn}\left(\left[\mathrm{ZZ}_{n}\right]\right)
$$

For some purposes, it may be useful to have a form of recursion relation that is intermediate between the twistorial (7.21) and ambidextrous (7.23), and involves the fewest integrals. Such a form may be obtained from (7.21) by Fourier transforming $\mathrm{W}_{n} \rightarrow \mathrm{Z}_{n}$, 
but not Fourier transforming the intermediate state. One finds

$$
\begin{aligned}
\mathcal{M}\left(\mathrm{W}_{1}, \ldots, \mathrm{Z}_{n}\right)=\sum \int D^{3 \mid \mathcal{N}} \mathrm{W} \mathcal{M}_{L}\left(\mathrm{~W}_{1}, \ldots, \mathrm{W}\right) & \\
\times & \operatorname{sgn}\left(\left\langle\mathrm{W}_{1} \mathrm{~W}\right\rangle \mathrm{Z}_{n} \cdot \mathrm{W}_{1} I \mathrm{Z}_{n} \cdot \frac{\partial}{\partial \mathrm{W}}\right) \mathcal{M}_{R}\left(\mathrm{~W}, \ldots, \mathrm{Z}_{n}\right) \\
=\sum \int \frac{\mathrm{d} t}{t} & D^{3 \mid \mathcal{N}} \mathrm{W} \mathcal{M}_{L}\left(\mathrm{~W}_{1}, \ldots, \mathrm{W}\right) \\
\times & \operatorname{sgn}\left(\left\langle\mathrm{W}_{1} \mathrm{~W}\right\rangle \mathrm{Z}_{n} \cdot \mathrm{W}_{1}\right) \mathcal{M}_{R}\left(\mathrm{~W}+t I \mathrm{Z}_{n}, \ldots, \mathrm{Z}_{n}\right),
\end{aligned}
$$

where $\left.I Z_{n}:=I_{\alpha \beta} Z_{n}^{\alpha}=\mid n\right]$.

\subsection{Ambidextrous form of the 3 point amplitudes}

To begin the recursion using the ambidextrous form (7.23), we need the three-point amplitudes written in an ambidextrous way. The SYM 3-point amplitudes

$$
\begin{aligned}
& \mathcal{A}_{\mathrm{MHV}}\left(\mathrm{W}_{1}, \mathrm{~W}_{2}, \mathrm{~W}_{3}\right)=\operatorname{sgn}\left(\left\langle\mathrm{W}_{2} \mathrm{~W}_{3}\right\rangle \mathrm{iW}_{2} \cdot \frac{\partial}{\partial \mathrm{W}_{1}} \mathrm{iW}_{3} \cdot \frac{\partial}{\partial \mathrm{W}_{1}}\right) \delta^{(4 \mid 4)}\left(\mathrm{W}_{1}\right) \\
& \mathcal{A}_{\overline{\mathrm{MHV}}}\left(\mathrm{W}_{1}, \mathrm{~W}_{2}, \mathrm{~W}_{3}\right)=\operatorname{sgn}\left(\left[\frac{\partial}{\partial \mathrm{W}_{2}} \frac{\partial}{\partial \mathrm{W}_{3}}\right] \mathrm{iW}_{1} \cdot \frac{\partial}{\partial \mathrm{W}_{2}} \mathrm{iW}_{1} \cdot \frac{\partial}{\partial \mathrm{W}_{3}}\right) \delta^{(4 \mid 4)}\left(\mathrm{W}_{2}\right) \delta^{(4 \mid 4)}\left(\mathrm{W}_{3}\right)
\end{aligned}
$$

become simply

$$
\begin{gathered}
\mathcal{A}_{\mathrm{MHV}}\left(\mathrm{Z}_{1}, \mathrm{~W}_{2}, \mathrm{~W}_{3}\right)=\operatorname{sgn}\left(\left\langle\mathrm{W}_{2} \mathrm{~W}_{3}\right\rangle \mathrm{Z}_{1} \cdot \mathrm{W}_{2} \mathrm{Z}_{1} \cdot \mathrm{W}_{3}\right) \\
\mathcal{A}_{\overline{\mathrm{MHV}}}\left(\mathrm{W}_{1}, \mathrm{Z}_{2}, \mathrm{Z}_{3}\right)=\operatorname{sgn}\left(\left[\mathrm{Z}_{2} \mathrm{Z}_{3}\right] \mathrm{Z}_{2} \cdot \mathrm{W}_{1} \mathrm{Z}_{3} \cdot \mathrm{W}_{1}\right)
\end{gathered}
$$

after representing the delta functions by their Fourier transforms. Similarly, the 3-point gravity amplitudes are

$$
\begin{aligned}
\mathcal{M}_{\mathrm{MHV}}\left(\mathrm{Z}_{1}, \mathrm{~W}_{2}, \mathrm{~W}_{3}\right) & =\left|\left\langle\mathrm{W}_{2} \mathrm{~W}_{3}\right\rangle\left(\mathrm{Z}_{1} \cdot \mathrm{W}_{2}\right)\left(\mathrm{Z}_{1} \cdot \mathrm{W}_{3}\right)\right| \\
\mathcal{M}_{\overline{\mathrm{MHV}}}\left(\mathrm{W}_{1}, \mathrm{Z}_{2}, \mathrm{Z}_{3}\right) & =\left|\left(\mathrm{W}_{1} \cdot \mathrm{Z}_{2}\right)\left(\mathrm{W}_{1} \cdot \mathrm{Z}_{3}\right)\left[\mathrm{Z}_{2} \mathrm{Z}_{3}\right]\right|
\end{aligned}
$$

Using (7.26) or (7.27) and the ambidextrous BCFW relation (7.23), one can build up arbitrary tree amplitudes on products of twistor and dual twistor spaces. The amplitudes are, in the first instance, expressed as integrals of these 3-point amplitudes, and make contact with Hodges' twistor diagrams [40, 43-45]. In the case of SYM, the integrands are simply products of sgn factors. Further applications of the ambidextrous form of the recursion relations can be found in [46].

Clearly, there is a direct correspondence between the calculations required to solve the recursion relations on dual twistor space and those required on ambitwistor space, but we will not expand on this here. In section 5.1, we showed that on dual twistor space, an arbitrary $n$-point $\mathrm{N}^{k} \mathrm{MHV}$ tree amplitude involves $2(n-2)$ Hilbert transforms and $(n-2-k)$ delta functions. Representing each of these $(n-2-k)$ delta functions as a Fourier transform, the Hilbert transforms (and other non-local sign operators) act under the integrals to yield a local function of the $\mathrm{Z}_{i} \mathrm{~s}$ (for $i=1, \ldots, n-2-k$ ) and the remaining Ws. Conversely, it is also possible to transform the result of an ambidextrous calculation onto (multiple copies of) dual twistor space by the reverse procedure. 


\section{Conclusions and outlook}

We have shown that the half Fourier transform provides a practical and coherent scheme for translating scattering amplitudes for massless field theories into twistor space. The BCFW recursion relations can be reformulated in a useful form and can in principle be used to generate the full tree sector of $\mathcal{N}=4$ super Yang-Mills theory and $\mathcal{N}=8$ supergravity. In practice, we have show that the twistor version of BCFW recursion is tractable, and have solved them for small $k \mathrm{~N}^{k} \mathrm{MHV}$ and googly $\mathrm{N}^{k} \mathrm{MHV}$ amplitudes. In general we have seen that, up to some 'signs', a $\mathrm{N}^{k} \mathrm{MHV}$ amplitude is expressed on twistor space and an intgegral over $2 n-4$ bosonic parameters (expressed here in the form of Hilbert tranforms) of $n-k-2$ delta functions $\delta^{4 \mid 4}$ of linear combinations of the $n$ twistors involved. As far as loop amplitudes are concerned, in principle there is no difficulty expressing them on twistor space via the half Fourier transform, and we showed that for the four-particle amplitude, this is actually straightforward. It is likely that the generalised unitarity methods $[6-9,14]$ that are so successful for constructing loop amplitudes for supersymmetric gauge theories can also be adapted to provide a generating principle for loop amplitudes on twistor space. ${ }^{23}$

Part of the motivation for this work was to express superconformal invariance more clearly. In fact, the formalism has made transparent that superconformal invariance is actually broken by factors of $\operatorname{sgn}(\langle 12\rangle\langle 23\rangle\langle 31\rangle)$ and the non-local operator $\operatorname{sgn}\left[\partial_{\mathrm{W}_{i}} \partial_{\mathrm{W}_{j}}\right]$. There is complete cancellation of these factors in even-point MHV and $\overline{\mathrm{MHV}}$ amplitudes (which are therefore conformally invariant), but there is a topological obstruction to making odd-point MHV amplitudes conformally invariant, even at tree-level. This may simply be reflecting a basic feature of scattering theory: to define an S-matrix, one must first choose an asymptotic region in which the particles are free. Indeed, such a choice is necessary to define $\operatorname{sgn}(\langle 12\rangle\langle 23\rangle\langle 31\rangle)$, for example. Choosing a light-cone to remove from conformally compactified space-time in order to define the amplitudes is analogous to choosing a cut of the complex $z$-plane in order to define $\sqrt{z}$. It seems also likely that these conformal symmetry breaking sign factors are an artifact of split signature as the obstructions to conformal symmetry only apply there (see also the arguments below).

However, the topological obstruction identified above cannot apply in Lorentz signature, because twistor lines are then $\mathbb{C P}^{1} \mathrm{~s}$ rather than $\mathbb{R} \mathbb{P}^{1} \mathrm{~s}$, and there is no notion of ordering points on a sphere. It is thus seems to be the case that the awkward signs and corresponding violation of conformal invariance is a consequence of our use of split signature. Support for this point of view comes from the fact that in complex twistor space, space-time fields correspond to cohomology classes, rather than by functions. Choosing a Dolbeault representation of the fields, the required antisymmetry of the kinematic part of the amplitude comes naturally from the wedge product of forms, as in the holomorphic Chern-Simons vertex

$$
\int_{\mathbb{C P}^{3 \mid 4}} D^{3 \mid 4} \mathrm{~W} \wedge \operatorname{tr}(A \wedge A \wedge A)
$$

\footnotetext{
${ }^{23}$ See $[54,56-59]$ for subsequent developments in this direction
} 
rather than from an explicit factor of $\operatorname{sgn}(\langle 12\rangle\langle 23\rangle\langle 31\rangle)$. To adapt this Chern-Simons vertex (or indeed the complete twistor actions of [26, 27, 29], that also rely on a Dolbeault description) to the split signature context, one must choose Dolbeault representatives defined from the X-ray data, such as those in [77]. These representatives are conformally invariant, but one must then choose an open cover to reduce the integrals from the full complex twistor space to overlaps of open sets, and eventually down to the real twistor space. These choices of open sets will break conformal invariance. Conversely, the presence of conformal breaking factors in the real twistor space amplitudes is perhaps required in order for them to have a cohomological form which may be analytically continued to Lorentz signature.

Certainly, in order to make the twistor formulation self-contained, one should really understand how the twistor recursion relations and seed amplitudes presented here can be obtained from the twistor actions. Since the twistor action for Yang-Mills is itself conformally invariant, the conformal breaking factors must arise either from gauge fixing or from a choice of Čech cover to give split signature X-ray representatives. It remains an open question as to whether one can introduce a twistor action that is itself naturally adapted either to a Čech description of cohomology, or to the split signature X-ray transform framework used in this paper. ${ }^{24}$

However they are viewed, for us the practical consequence of breaking conformal invariance is simply that the factors of $\operatorname{sgn}\left(\left\langle\mathrm{W}_{i} \mathrm{~W}_{j}\right\rangle\right)$ and particularly the non-local operator $\operatorname{sgn}\left(\left[\partial_{\mathrm{W}_{i}} \partial_{\mathrm{W}_{j}}\right]\right)$ make the twistor $\mathrm{BCFW}$ recursion more complicated. It is tempting to speculate whether there could perhaps be a sense in which they can be discarded, maybe as a model for a fully complex BCFW recursion. For example, consider the gluing rule

$$
\mathcal{A}^{\prime}(1, \ldots, n)=\sum \int \frac{\mathrm{d} t}{|t|} D^{3 \mid 4} \mathrm{~W} \mathcal{A}_{L}^{\prime}\left(\mathrm{W}_{1}, \ldots, \mathrm{W}\right) \mathcal{A}_{R}^{\prime}\left(\mathrm{W}, \ldots, \mathrm{W}_{n}-t \mathrm{~W}_{1}\right)
$$

in real twistor space. Unlike BCFW recursion, this gluing rule is (manifestly) superconformally invariant as well as projective. If we seed (8.1) by the three-point objects

$$
\begin{aligned}
& \mathcal{A}_{\mathrm{MHV}}^{\prime}(1,2,3)=\delta^{(2 \mid 4)}\left(\mathrm{W}_{1}, \mathrm{~W}_{2}, \mathrm{~W}_{3}\right) \\
& \mathcal{A}_{\overline{\mathrm{MHV}}}^{\prime}(1,2,3)=\delta^{(3 \mid 4)}\left(\mathrm{W}_{1}, \mathrm{~W}_{2}\right) \delta^{(3 \mid 4)}\left(\mathrm{W}_{1}, \mathrm{~W}_{3}\right)
\end{aligned}
$$

then the recursion procedure is manifestly superconformally invariant. ${ }^{25}$ The output of this recursion then seems to produce purely geometric sums of products of delta functions, just

\footnotetext{
${ }^{24}$ Similar questions concern the relation of the twistor diagram approach of Hodges [43-45] and ArkaniHamed et al. [46] to the ambitwistor action [64]. Preliminary calculations show that [43-46] are working with the Feynman rules of the ambitwistor action on "the momentum space of ambitwistor space" rather than on ambitwistor space itself.

${ }^{25}$ In split signature, recursion using (8.1) seeded by (8.2) corresponds on momentum space to recursion using the rule

$$
A^{\prime}(1, \ldots, n)=\sum A_{L}^{\prime}(\hat{1}, \ldots,-\hat{p}) \frac{1}{\left|p_{L}^{2}\right|} A_{R}^{\prime}(\hat{p}, \ldots, \hat{n})
$$

seeded by the three-point objects

$$
\begin{aligned}
& A_{\mathrm{MHV}}^{\prime}(1,2,3)=\frac{\delta^{(4 \mid 8)}\left(\sum_{i=1}^{3}|i\rangle \llbracket i \|\right)}{|\langle 12\rangle\langle 23\rangle\langle 31\rangle|} \\
& A_{\overline{\mathrm{MHV}}}^{\prime}(1,2,3)=\frac{\delta^{(4)}\left(\sum_{i=1}^{3}|i\rangle[i \mid) \delta^{(0 \mid 4)}\left(\eta_{1}\langle 23\rangle+\eta_{2}\langle 31\rangle+\eta_{3}\langle 12\rangle\right)\right.}{|[12][23][31]|}
\end{aligned}
$$


as one might obtain by manually removing the conformal-breaking signs from amplitudes and replacing the sgn in the remaining $\operatorname{sgn}\left(\mathrm{W}_{i} \cdot \partial_{j}\right)$ operators by logs (see footnote 10 in section 3.1.1). Although (8.1) \& (8.2) have the wrong exchange properties to be amplitudes, it is perhaps conceivable that the true split signature amplitudes can be recovered from this simpler recursion rule by dressing it with conformal-breaking signs once the recursion has been performed. One might also speculate that a Dolbeault version of BCFW recursion in complex twistor space bears more resemblance to (8.1) than to the actual split signature BCFW rule, with the exchange properties coming from understanding the (seed) amplitudes as forms, rather than (delta-)functions. As with the twistor actions, the conformalbreaking sign factors in the true split signature BCFW rule might then arise from choosing representatives for these forms with respect to a cover that is adapted to real twistor space.

An alternative to making contact with twistor actions is to make contact with the MHV formalism form. It is possible to start from Risager's shift [65] (or its multiline extensions $[66,67]$ ) and proceed as in this paper, obtaining a twistor representation of the MHV formalism [3] in split signature. This has some advantages, but the big disadvantage that conformal (and indeed Lorentz) invariance is expressly broken by the choice of spinor used in the gauge choice and this in the long run leads to more complicated formulae.

A more interesting approach might be to work with twistor-string theory [1], which again deals with on-shell amplitudes. The connected prescription computes an $\mathrm{N}^{k} \mathrm{MHV}$ super-amplitude by means of an integral [2] over the moduli space $\bar{M}_{0, n}\left(\mathbb{C P}^{3 \mid 4}, d\right)$ of $n$ pointed, degree $d$ (stable) maps

$$
f:\left(\Sigma ; p_{1}, \ldots, p_{n}\right) \rightarrow \mathbb{C P}^{3 \mid 4},
$$

where $d=k+1$ (see e.g. [78] for an introduction of the bosonic part of this moduli space, and [79] for a discussion in the context of twistor-strings). This space has bosonic dimension $4 d+n$. If the vertex operators are constructed from elemental states, they restrict $f$ to take each of the marked points $p_{i}$ (insertion points of the vertex operators) on $\Sigma$ to a specific point $Z_{i} \in \mathbb{C P}^{3 \mid 4}$. This implies three bosonic constraints per vertex operator, so the space of such maps has virtual dimension $-2 n+4 d=-2(n-2 k-2)$. The implication of this virtual dimension being negative is simply that the points $Z_{i}$ cannot be in general position in $\mathbb{C P}^{3}$, and the amplitude only has support on some algebraic subset. Specifically, there are $2(n-2 k-2)$ constraints on the locations of the points in twistor space. This fits in precisely with the counting of bosonic delta functions in section 5.1, where we found that an $\mathrm{N}^{k} \mathrm{MHV} 4(n-2-k)$ delta-functions, dressed by $2(n-2)$ Hilbert transforms, each of which soaks up a delta function. It would be interesting to see how the corresponding parametrisation of the moduli space arises.

One might then hope to understand the BCFW recursion formula in the context of the connected prescription by considering the one-parameter family of amplitudes $\mathcal{A}\left(Z_{1}, \ldots, Z_{n-1}, Z_{1}-t Z_{n}\right)$ where the marked point $p_{n}$ is only required to be mapped to 
the line joining $Z_{1}$ to $Z_{n}$. The space of such maps has dimension 1 greater than the support of $\mathcal{A}\left(Z_{1}, \ldots, Z_{n-1}, Z_{n}\right)$, and one could seek a derivation of the twistor BCFW rule by understanding how this curve interacts with the boundary divisors in $\bar{M}_{0, n}\left(\mathbb{C P}^{3 \mid 4}, d\right)$, corresponding to degenerations of $\left(\Sigma ; p_{1}, \ldots, p_{n}\right)$ to a nodal curve. This would make contact with the work of Vergu [80] on the factorisation limits of the connected prescription. ${ }^{26}$

A major attraction of reformulating these ideas in connection with twistor-string theory is the possibility of realising Witten's proposal [1] that the Yangian symmetry of planar $\mathcal{N}=4$ super Yang-Mills, seen at both weak [31, 81] and strong [82, 83] coupling, should arise from the harmonic map equations underlying the twistor-string.

\subsection{Signature signs and loops}

In the discussion of loops, the problems that arise from working with split signature become sharper. When one analytically continues to Euclidean signature, the structure of the Feynman propagator implies a unique continuation. However, the Feynman prescription requires a concept of time ordering that makes no sense in split signature; in split signature the lightcone is connected and does not naturally divide into past and future. It would therefore be problematic to be restricted to considering scattering amplitudes in split signature.

Our view is that the split signature methods developed in this paper (and those of [46]) represent a clear route to discovering underlying twistorial structures of amplitudes that will also have a direct reformulation that makes sense in the complex and, in particular, in Lorentz signature. A model for how this is likely to work is provided by twistor diagram theory which has never been restricted to any particular signature. In this approach, the integrals are all considered to be contour integrals defined in complex twistor space.

Some aspects of the translation procedure into the complex are straightforward. Wave functions that are simply functions of the real twistors should be required to have an analytic extension becoming holomorphic functions on certain open sets. These would then be re-interpreted as Cech cocycles as described in [42, 77, 85-89]. With the reformulation as Cech cocycles, the signs in the formula would no longer appear explicitly, but be encoded into the cohomology class containing the information of which overlaps in some open cover the Cech cocycles are associated with. The integrals can then be understood as contour integrals that are homological to the real slice. Where, in our formalism we have used a delta function $\delta(z)$, in the complex, we would replace this with the pole $1 / 2 \pi i z$ and specify that the topology of the integration contour should be specified so that the integration can be performed by a residue calculation.

The above discussion indicates that in such a complexified reformulation, the awkward signs should be suppressed from explicit formulae, although their presence should nevertheless be felt when it comes to specifying the contour. Indeed precisely these ingredients, including many details, play a role in the correspondence between the work of [46] and Andrew Hodges' twistor diagrams.

There are of course many technical obtstructions to overcome to make this programme precise. Perhaps the most difficult aspect of twistor diagram theory is the correct cohomo-

\footnotetext{
${ }^{26}$ See [58] for the correct explanation of these relationships
} 
logical interpretation. This is rendered particularly problematic by the infrared divergences that are present even at tree level. These mean that one doesnt necessarily expect the integrals to make good sense even in the simplest examples. Another related issue is the systematic specification of the contour.

An important role in our formulation of a twistor amplitude is the concept of an elemental state (i.e., in the real context, this is a twistor function on real twistor space that has delta function support at a point, or, in Fourier transformed form, one of the form $\operatorname{sgn}(Z \cdot A)$ or $\log (Z \cdot A))$. These are essentially the external states that are being scattered in a twistor amplitude expression. Analogues of such states were originally defined in the complex [75], but their cohomological interpretation remains elusive. These are used to give us the integral kernel of the amplitude, and its not so clear that they should have a standard interpretation as an external wave-function. It is reasonable to expect that kernels of scattering amplitudes should have a different cohomological status to that of wave functions as one expects them to have different analyticity properties; massless wavefunctions are arbitrary smooth functions on the momentum light-cone, while tree amplitudes are meromorphic functions on the complexified lightcone in momentum space. Under the half Fourier transform, analyticity in momentum space corresponds to twistor amplitudes that have restricted support. In Lorentz signature, crossing symmetry requires the amplitudes be well-defined when integrated against external states of both positive and negative frequency. In twistor space, Lorentzian wavefunctions of positive and negative frequency are represented by elements of $H^{1}\left(\overline{\mathbb{P T}^{+}}, \mathcal{O}(-2 h-2)\right)$ and $H^{1}\left(\overline{\mathbb{P T}^{-}}, \mathcal{O}(-2 h-2)\right)$, respectively (see e.g. [76]), where $\overline{\mathbb{P T}^{+}}$is the region of complex twistor space for which the $\mathrm{SU}(2,2)$ inner product $Z \cdot \bar{Z} \geq 0$, while $\overline{\mathbb{P T}^{-}}$has $Z \cdot \bar{Z} \leq 0$. In order to be able to pair a scattering amplitude with either of these, the amplitude's support must be contained in $\mathbb{P N}=\overline{\mathbb{P T}^{+}} \cap \overline{\mathbb{P T}^{-}}$. It is suggestive that this can be made to be satisfied by our (split signature) formulae if we choose an $\mathbb{R P}^{3}$ real slice that lies inside $\mathbb{P N}$ (of which there are many). Thus the formulae we have obtained may serve perfectly well to define amplitudes in Lorentz signature at least at tree level.

There have not yet been sufficiently many loop calculations performed to get a feel for how a complex formulation should work at loops. The calculations of section 5.7 show that one can obtain a twistor amplitude for each choice of analytic continuation from Lorentz signature through to split signature. However, there are many choices for fixing the ambiguity in the continuation of such a loop amplitude around its branching singularities. In [46] the ambiguity in loop diagrams was resolved essentially by using a propagator that was based on the principal value regularization, rather than the Feynman $i \epsilon$. They obtained the answer ' 1 ' for this amplitude. For us, this answer is singular as one must take a Fourier transform to obtain expressions on twistor space alone. It was there proposed that this answer should be related to the physical Lorentzian signature amplitude by analytic continuation back to Lorentz signature and being careful to pick up the contributions from the singularities that one passes en-route. According to the ideas outlined above, the final correct answer will be most directly expressed as a contour integral in the complex. However to get a well-defined answer we will also need to incorporate some satisfactory form of regularization. Andrew Hodges has already introduced a regularization procedure 
in the twistor diagram formulation that seems to be sufficient for the task at tree-level. It will be interesting to see whether this can used to make sense of loop amplitudes also.

\section{Acknowledgments}

It is a pleasure to thank N. Arkani-Hamed, R. Boels, R. Britto, F. Cachazo, C. Cheung, J. Drummond, J. Henn, A. Hodges, J. Kaplan, G. Korchemsky, D. Kosower and E. Sokatchev for many useful comments and discussions. We would also like to thank the organisers of the conference "Hidden Structures in Field Theory Amplitudes" at NBIA in September 2008 where the ideas for this paper were sown, as well as the faculty and staff of both Perimeter Institute and IHÉS where this work was partially carried out. LM is supported in part by the EU through the FP6 Marie Curie RTN ENIGMA (contract number MRTN-CT-2004-5652) and through the ESF MISGAM network. This work was financed by EPSRC grant number EP/F016654, see also http://gow.epsrc.ac.uk/ViewGrant.aspx?GrantRef=EP/F016654/1.

\section{A Conventions, notation and background}

For ease of comparison to the scattering theory literature (in which an MHV amplitude has two negative and an arbitrary number of positive helicity states), we will focus on dual twistor space. We work throughout with real (dual) twistors, so as to use Witten's half Fourier transform, and correspondingly our space-time signature is $(++--)$. Dual twistor space, $\mathbb{P T}^{*}$ will therefore be a copy of real projective 3 -space, $\mathbb{R P}^{3}$ with homogeneous co-ordinates $\left[W_{\alpha}\right]=\left[\lambda_{A}, \mu^{A^{\prime}}\right]$, where $A$ and $A^{\prime}$ denote anti-selfdual and self-dual two-component spinor indices, respectively. Twistor space $\mathbb{P T}$ has homogeneous co-ordinates $Z^{\alpha}=\left(\omega^{A}, \pi_{A^{\prime}}\right)$.

The conformal group in this signature is $\operatorname{PSL}(4, R)$ acting linearly on the homogeneous twistor co-ordinates. To break the symmetry down to the Poincare group, we introduce the infinity twistors $I^{\alpha \beta}$ or $I_{\alpha \beta}$. These are defined so that for a twistor $Z^{\alpha}=\left(\omega^{A}, \pi_{A^{\prime}}\right)$ and dual twistor $W_{\alpha}=\left(\lambda_{A}, \mu^{A^{\prime}}\right)$ we have

$$
I^{\alpha \beta}=\frac{1}{2} \varepsilon^{\alpha \beta \gamma \delta} I_{\gamma \delta}, \quad Z^{\alpha} I_{\alpha \beta}=\left(0, \pi^{A^{\prime}}\right) \quad I^{\alpha \beta} W_{\beta}=\left(\lambda^{A}, 0\right) .
$$

The equation $I^{\alpha \beta} W_{\beta}=0$ gives the line in dual twistor space corresponding to the point at infinity in Minkowski space, and the scale of $I^{\alpha \beta}$ fixes the space-time metric via the spinor inner products below.

We often use the spinor helicity notation

$$
\langle\alpha \beta\rangle:=\alpha_{A} \beta^{A}, \quad[\tilde{\alpha} \tilde{\beta}]:=\tilde{\alpha}^{A^{\prime}} \tilde{\beta}_{A^{\prime}} \quad \text { and } \quad \alpha_{A} U^{A A^{\prime}} \tilde{\beta}_{A^{\prime}}:=\langle\alpha|U| \tilde{\beta}]
$$

for inner products of spinors. On twistor space, the Poincaré invariant inner products are

$$
\begin{gathered}
\left\langle W_{1} W_{2}\right\rangle:=I^{\alpha \beta} W_{1 \alpha} W_{2 \beta}=\lambda_{1 A} \lambda_{2}^{A}=\langle 12\rangle \\
{\left[Z_{1} Z_{2}\right]:=I_{\alpha \beta} Z_{1}^{\alpha} Z_{2}^{\beta}=\pi_{1}^{A^{\prime}} \pi_{2 A^{\prime}}=[12]}
\end{gathered}
$$


The null cone $p^{2}=0$ in momentum space may be co-ordinatised by $p_{A A^{\prime}}=\lambda_{A} \tilde{\lambda}_{A^{\prime}}$. In split signature $\lambda_{A}$ and $\tilde{\lambda}_{A^{\prime}}$ are each real, independent, two-component spinors defined up to the scaling $\left(\lambda_{A}, \tilde{\lambda}_{A^{\prime}}\right) \rightarrow\left(r \lambda_{A}, r^{-1} \tilde{\lambda}_{A^{\prime}}\right)$ for $r$ any non-zero real number. A wavefunction $\Phi\left(\lambda_{A}, \tilde{\lambda}_{A^{\prime}}\right)$ on the light-cone in momentum space can be related to a (dual) twistor function $f(W)$ by Witten's 'half Fourier transform'

$$
f(W)=\int \mathrm{d}^{2} \tilde{\lambda} \mathrm{e}^{\mathrm{i} \mu^{A^{\prime}} \tilde{\lambda}_{A^{\prime}}} \Phi(\lambda, \tilde{\lambda}) ; \quad \Phi(\lambda, \tilde{\lambda})=\frac{1}{(2 \pi)^{2}} \int \mathrm{d}^{2} \mu \mathrm{e}^{-\mathrm{i} \mu A^{A^{\prime}} \tilde{\lambda}_{A^{\prime}}} f(\mu, \lambda)
$$

which makes sense only when $\tilde{\lambda}_{A^{\prime}}$ and $\mu^{A^{\prime}}$ are real.

In discussing supersymmetric theories, we use on-shell supermultiplets such as the $\mathcal{N}=4$ Yang-Mills supermultiplet in the $\eta$-representation

$$
\Phi(\lambda, \tilde{\lambda}, \eta)=G^{+}(\lambda, \tilde{\lambda})+\eta^{a} \Gamma_{a}(\lambda, \tilde{\lambda})+\cdots+\frac{1}{4 !} \epsilon_{a b c d} \eta^{a} \eta^{b} \eta^{c} \eta^{d} G^{-}(\lambda, \tilde{\lambda}) .
$$

Here $G^{ \pm}$are the on-shell momentum space wavefunctions of the helicity \pm 1 parts of the multiplet etc., and $\eta^{i}$ are Grassmann co-ordinates on the on-shell momentum superspace. A supersymmetric half Fourier transform

$$
f(\lambda, \mu, \chi)=\int \mathrm{d}^{2} \tilde{\lambda} \mathrm{d}^{\mathcal{N}} \eta \mathrm{e}^{\mathrm{i}\left(\mu^{A^{\prime}} \tilde{\lambda}_{A^{\prime}}+\chi_{i} \eta^{i}\right)} \Phi(\lambda, \tilde{\lambda}, \eta)
$$

relates this to a supertwistor multiplet. We often write

$$
\llbracket \mu \tilde{\lambda} \rrbracket:=\mu^{A^{\prime}} \tilde{\lambda}_{A^{\prime}}+\chi_{a} \eta^{a}, \quad \mathrm{~d}^{2 \mid \mathcal{N}} \tilde{\lambda}:=\mathrm{d}^{2} \tilde{\lambda} \mathrm{d}^{\mathcal{N}} \eta, \quad \mathrm{d}^{2 \mid \mathcal{N}} \mu:=\mathrm{d}^{2} \mu \mathrm{d}^{\mathcal{N}} \chi
$$

for these commonly occurring supersymmetric combinations. We denote the homogeneous co-ordinates on (dual) supertwistor space $\mathbb{R} \mathbb{P}^{3 \mid \mathcal{N}^{*}}$ by $\mathrm{W}=\left(W_{\alpha}, \chi_{a}\right)=\left(\lambda_{A}, \mu^{A^{\prime}}, \chi_{a}\right)$.

\section{B The X-ray and half Fourier transforms}

In this appendix we will examine the relation of Witten's half Fourier transform [1]

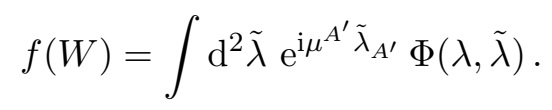

to the usual Penrose transform [39]

$$
\phi(x)=\left.\oint\langle\lambda \mathrm{d} \lambda\rangle f(W)\right|_{\mu^{A^{\prime}}=-x^{A A^{\prime}} \lambda_{A}}
$$

and its generalisations to other helicities.

In much of the twistor literature, space-time has either the physical, Lorentzian signature, or has Euclidean signature, or else is treated as complex. For (conformally) flat space-times, twistor space is then complex projective three-space $\mathbb{C P}^{3}$. The half Fourier transform does not apply in these contexts. Instead, massless fields are canonically related to cohomology classes (either Čech or Dolbeault) in twistor space via the abstract 
Penrose transform [76]. The concrete Penrose integral formula (B.2) involves a representative $f$ of this cohomology classes. However, there is gauge freedom inherent in picking such a representative, and this shows itself in (B.2) because (in the Čech picture) one can add to $f(W)$ any function whose singularities are not separated by the contour without changing the space-time field $\phi(x)$. In the Dolbeault picture $f$ should be thought of as a $(0,1)$-form and (B.2) interpreted as an integral over the full Riemann sphere. Again, adding any $\bar{\partial}$-exact piece to $f$ does not change $\phi(x)$. Furthermore, to even pick a Dolbeault representatives in the first place one needs to solve a $\bar{\partial}$-equation, whilst to pick a Čech representative one must first specify a covering of twistor space. Thus, in Lorentzian or Euclidean space-time, the twistor representation is rather subtle.

However, in $(++--)$ signature space-time, the twistor representation becomes much simpler: Twistor space is now real projective three-space and fields are represented in terms of straightforward functions. This simplicity has been our main reason for working with split signature in this paper. See e.g. [84] for a detailed discussion of twistor theory in $(++--)$ signature space-time.

As pointed out by Atiyah in [42], in (++--) signature, the Penrose transform (B.2) in its Čech format can be reinterpreted as the 'X-ray transform' of Fritz John [41]. The X-ray transform ${ }^{27}$ takes a function $f$ on $\mathbb{R}^{3}$ and transforms it to a function $\phi$ on the fourdimensional space of directed lines in $\mathbb{R}^{3}$ given by integration along corresponding line in $\mathbb{R}^{3}$. It naturally extends to a map from functions on $\mathbb{P T}=\mathbb{R} \mathbb{P}^{3}$ to the space $\widetilde{M}$ of oriented lines in $\mathbb{P} T$. On dual twistor space (to fit with the scattering theory community) it can be expressed on $\mathbb{P T}^{\prime *}=\mathbb{P T}^{*}-\left\{\lambda_{A}=0\right\}$ by

$$
\phi(x):=\oint_{L_{x}}\langle\lambda \mathrm{d} \lambda\rangle f(W),
$$

where $L_{x}$ is the line in twistor space given by the incidence relation

$$
\mu^{A^{\prime}}=-x^{A A^{\prime}} \lambda_{A}
$$

for $x$ in $(2,2)$ signature space-time $\mathbb{M}$, and $f$ is an arbitrary smooth function on real twistor space $\mathbb{P} T$. Fritz John showed that $\phi$ satisfies the wave equation and that, under suitable assumptions, the X-ray transform is an isomorphism. There has by now been much work over the years to understand how the X-ray transform and its relatives fit into the Penrose transform [77, 85-89] and its non-linear analogues [26, 90-92]. Early proofs of invertibility followed by expanding $f(Z)$ in spherical harmonics, where the X-ray transform integral may be done explicitly, and then using the completeness relations of the spherical harmonics. Subsequent proofs used complex analysis and adapted versions of the Penrose transform. We now give an alternative proof that also gives a proof of Witten's half Fourier transform.

\section{B.1 Scalar fields}

For ease of comparison to the scattering theory literature, we will focus on the transform from dual twistor space $\mathbb{P T}^{*}=\mathbb{R P}^{3 *}$ with real co-ordinates $W_{\alpha}=\left(\lambda_{A}, \mu^{A^{\prime}}\right)$. The dual

\footnotetext{
${ }^{27}$ It gets its name from X-ray imaging, where $f$ is taken to be the density of the body to be X-rayed, and the integral of $f$ along lines gives the attenuation of the X-ray as it passes through.
} 
twistor X-ray transform for the scalar wave equation is

$$
\phi(x)=\int\langle\lambda \mathrm{d} \lambda\rangle f\left(\lambda,-x^{A A^{\prime}} \lambda_{A}\right),
$$

where $f(W)$ is an arbitrary function of homogeneity -2 (so $f(r W)=r^{-2} f(W)$ ). Differentiating under the integral sign, it is easy to check that any scalar field constructed via (B.5) automatically obeys the massless field equation $\square \phi(x)=0$. In fact, it will follow from the argument below that all such fields may be constructed this way. Hence, the Fourier transform $\tilde{\phi}(p)$ satisfies $p^{2} \tilde{\phi}(p)=0$, and so

$$
\tilde{\phi}(p)=\delta\left(p^{2}\right) \Phi(\lambda, \tilde{\lambda})
$$

for some function $\Phi(\lambda, \tilde{\lambda})$ defined on the null cone in momentum space, where $p_{A A^{\prime}}=\lambda_{A} \tilde{\lambda}_{A^{\prime}}$.

Combining the X-ray and Fourier transforms, we have

$$
\delta\left(p^{2}\right) \Phi(\lambda, \tilde{\lambda})=\int \mathrm{d}^{4} x \mathrm{e}^{-\mathrm{i} p \cdot x} \phi(x)=\int \mathrm{d}^{4} x \mathrm{e}^{-\mathrm{i} p \cdot x}\left\{\int\left\langle\lambda^{\prime} \mathrm{d} \lambda^{\prime}\right\rangle f\left(\lambda_{A}^{\prime},-x^{A A^{\prime}} \lambda_{A}^{\prime}\right)\right\}
$$

where $\left|\lambda^{\prime}\right\rangle$ is a dummy spinor variable. Now, because $f$ depends on $x$ only through the combination $x^{A A^{\prime}} \lambda_{A}^{\prime}$, half of the $x$-integrals may be performed directly. To do this, choose ${ }^{28}$ a constant spinor $|\alpha\rangle$ with $\left\langle\alpha \lambda^{\prime}\right\rangle \neq 0$ and decompose $x$ as

$$
x^{A A^{\prime}}=\frac{\mu^{A^{\prime}} \alpha^{A}-\chi^{A^{\prime}} \lambda^{\prime A}}{\left\langle\alpha \lambda^{\prime}\right\rangle},
$$

where $\mu^{A^{\prime}}=-x^{A A^{\prime}} \lambda_{A}^{\prime}$ and $\chi^{A^{\prime}}:=x^{A A^{\prime}} \alpha_{A}$. (Equation (B.8) is easily checked by contracting both sides with either $|\alpha\rangle$ or $\left|\lambda^{\prime}\right\rangle$.) The measure $\mathrm{d}^{4} x$ decomposes as

$$
\mathrm{d}^{4} x=\frac{\mathrm{d}^{2} \mu \mathrm{d}^{2} \chi}{\left\langle\alpha \lambda^{\prime}\right\rangle^{2}} .
$$

We now integrate out $|\chi\rangle$ to obtain

$$
\begin{aligned}
\delta\left(p^{2}\right) \Phi(\lambda, \tilde{\lambda}) & =\int \mathrm{d}^{4} x\left\langle\lambda^{\prime} \mathrm{d} \lambda^{\prime}\right\rangle \mathrm{e}^{-\mathrm{i} p \cdot x} f\left(\lambda^{\prime},-x\left|\lambda^{\prime}\right\rangle\right) \\
& =\int \frac{\mathrm{d}^{2} \mu \mathrm{d}^{2} \chi\left\langle\lambda^{\prime} \mathrm{d} \lambda^{\prime}\right\rangle}{\left\langle\alpha \lambda^{\prime}\right\rangle^{2}} \exp \left(-\mathrm{i} \frac{[\mu|p| \alpha\rangle-\left[\chi|p| \lambda^{\prime}\right\rangle}{\left\langle\alpha \lambda^{\prime}\right\rangle}\right) f\left(\lambda^{\prime}, \mu\right) \\
& =\int \frac{\mathrm{d}^{2} \mu\left\langle\lambda^{\prime} \mathrm{d} \lambda^{\prime}\right\rangle}{\left\langle\alpha \lambda^{\prime}\right\rangle^{2}} \delta^{2}\left(\frac{p_{A A^{\prime}} \lambda^{\prime A}}{\left\langle\alpha \lambda^{\prime}\right\rangle}\right) \mathrm{e}^{-\mathrm{i} \frac{[\mu|p| \alpha\rangle}{\left\langle\alpha \lambda^{\prime}\right\rangle}} f\left(\lambda^{\prime}, \mu\right) .
\end{aligned}
$$

The $\delta$-functions inside the integral may be converted into $\delta$-functions involving the integration variable $\lambda^{\prime}$ at the expense of a Jacobian

$$
\delta^{2}\left(\frac{p_{A A^{\prime}} \lambda^{\prime A}}{\left\langle\alpha \lambda^{\prime}\right\rangle}\right)=\left|\left\langle\alpha \lambda^{\prime}\right\rangle\langle\alpha \lambda\rangle\right| \delta\left(p^{2}\right) \delta\left(\left\langle\lambda \lambda^{\prime}\right\rangle\right),
$$

\footnotetext{
${ }^{28}$ The choice will soon be seen to drop out.
} 
where $p=\lambda \tilde{\lambda}$ on the support of $\delta\left(p^{2}\right)$. The $\mathrm{d} \lambda^{\prime}$ integral may now be performed, yielding finally

$$
\begin{aligned}
\delta\left(p^{2}\right) \Phi(\lambda, \tilde{\lambda}) & =\delta\left(p^{2}\right) \int \mathrm{d}^{2} \mu\left\langle\lambda^{\prime} \mathrm{d} \lambda^{\prime}\right\rangle\left|\frac{\langle\alpha \lambda\rangle}{\left\langle\alpha \lambda^{\prime}\right\rangle}\right| \delta\left(\left\langle\lambda \lambda^{\prime}\right\rangle\right) \mathrm{e}^{-\mathrm{i}[\mu \tilde{\lambda}] \frac{\langle\alpha \lambda\rangle}{\left\langle\alpha \lambda^{\prime}\right\rangle}} f\left(\lambda^{\prime}, \mu\right) \\
& =\delta\left(p^{2}\right) \int \mathrm{d}^{2} \mu \mathrm{e}^{-\mathrm{i}[\mu \tilde{\lambda}]} f(\lambda, \mu)
\end{aligned}
$$

which is precisely Witten's half Fourier transform [1]. Provided $\Phi$ is sufficiently wellbehaved, (B.12) can be inverted by standard Fourier analysis to give

$$
f(\lambda, \mu)=\int \mathrm{d}^{2} \tilde{\lambda} \mathrm{e}^{\mathrm{i}[\mu \tilde{\lambda}]} \Phi(\lambda, \tilde{\lambda})
$$

as is used throughout the text.

\section{B.2 Generalisation to other helicities}

Like the Penrose transform, the X-ray transform may be generalised to fields of helicity $h$. Specifically, on-shell massless fields of helicity $h$ are represented on real (dual) twistor space by functions of homogeneity $2 h-2$ and the X-ray transform is either

$$
\phi_{A B \cdots D}(x)=\left.\int\langle\lambda \mathrm{d} \lambda\rangle \lambda_{A} \lambda_{B} \cdots \lambda_{D} f_{2 h-2}(W)\right|_{\mu=-x \lambda}
$$

when $h<0$, or

$$
\phi_{A^{\prime} B^{\prime} \ldots D^{\prime}}(x)=\left.\int\langle\lambda \mathrm{d} \lambda\rangle \frac{\partial^{2 h}}{\partial \mu^{A^{\prime}} \partial \mu^{B^{\prime}} \cdots \partial \mu^{D^{\prime}}} f_{2 h-2}(W)\right|_{\mu=-x \lambda}
$$

when $h>0$. In particular, when $|h|=1$ they represent the anti-self-dual and self-dual parts of the linearized Yang-Mills field strength

$$
F_{A B A^{\prime} B^{\prime}}=\epsilon_{A^{\prime} B^{\prime}} \phi_{A B}(x)+\epsilon_{A B} \phi_{A^{\prime} B^{\prime}}(x)
$$

and when $|h|=2$ they likewise represent the self-dual and anti-self-dual parts of the linearized Weyl curvature

$$
W_{A B C D A^{\prime} B^{\prime} C^{\prime} D^{\prime}}(x)=\epsilon_{A^{\prime} B^{\prime}} \epsilon_{C^{\prime} D^{\prime}} \phi_{A B C D}(x)+\epsilon_{A B} \epsilon_{C D} \phi_{A^{\prime} B^{\prime} C^{\prime} D^{\prime}}(x) .
$$

Again, differentiating under the integral sign in (B.14) and (B.15), one verifies that that these fields automatically satisfy the linearized Yang-Mills or Einstein equations, which in spinor form are

$$
\nabla^{A A^{\prime}} \phi_{A B \cdots D}(x)=0 \quad \text { and } \quad \nabla^{A A^{\prime}} \phi_{A^{\prime} B^{\prime} \cdots D^{\prime}}(x)=0 .
$$

Since we are dealing with linearized curvatures, these formulæ are all gauge invariant.

For general helicity, the field equations (B.18) imply that the Fourier transformed fields obey

$$
p^{A A^{\prime}} \tilde{\phi}_{A B \cdots D}(p)=0, \quad p^{A A^{\prime}} \tilde{\phi}_{A^{\prime} B^{\prime} \cdots D^{\prime}}(p)=0 .
$$


Away from $p^{2}=0, p^{A A^{\prime}}$ is invertible. Hence the Fourier transforms take the form ${ }^{29}$

$$
\begin{aligned}
\tilde{\phi}_{A B \cdots D}(p) & =\mathrm{i}^{2 h} \delta\left(p^{2}\right) \lambda_{A} \lambda_{B} \cdots \lambda_{D} \Phi_{2 h}(\lambda, \tilde{\lambda}) \\
\tilde{\phi}_{A^{\prime} B^{\prime} \cdots D^{\prime}}(p) & =\delta\left(p^{2}\right) \tilde{\lambda}_{A^{\prime}} \tilde{\lambda}_{B^{\prime}} \cdots \tilde{\lambda}_{D^{\prime}} \Phi_{2 h}(\lambda, \tilde{\lambda})
\end{aligned}
$$

where as before $p_{A A^{\prime}}=\lambda_{A} \tilde{\lambda}_{A^{\prime}}$ and $\Phi_{2 h}(\lambda, \tilde{\lambda})$ must scale under $(\lambda, \tilde{\lambda}) \rightarrow\left(r \lambda, r^{-1} \tilde{\lambda}\right)$ as

$$
\Phi_{2 h}\left(r \lambda, r^{-1} \tilde{\lambda}\right)=r^{2 h} \Phi_{2 h}(\lambda, \tilde{\lambda})
$$

so as to balance the scaling of the spinor pre-factors (recall that $h<0$ for $\tilde{\phi}_{A B \cdots D}$ ). Equations (B.20) are fixed purely by kinematics, and hold irrespective of the particular wavefunction of the on-shell state. The wavefunction itself is determined by a choice of particular function $\Phi_{2 h}(\lambda, \tilde{\lambda})$.

The half Fourier transform for non-zero helicity has the same relation to the X-ray transform as it does for the scalar field treated above. When $h<0$, the same steps as in equations (B.7)-(B.12) give

$$
\tilde{\phi}_{A B \cdots D}(p)=\delta\left(p^{2}\right) \int \mathrm{d}^{2} \mu \mathrm{e}^{-\mathrm{i}[\mu \tilde{\lambda}]} \lambda_{A} \lambda_{B} \cdots \lambda_{D} f_{2 h-2}(W),
$$

whereas when $h>0$, the $\mathrm{d}^{2} \mu$ integral is done by parts to find similarly

$$
\begin{aligned}
\tilde{\phi}_{A^{\prime} B^{\prime} \cdots D^{\prime}}(p) & =\delta\left(p^{2}\right) \int \mathrm{d}^{2} \mu \mathrm{e}^{-\mathrm{i}[\mu \tilde{\lambda}]} \frac{\partial^{2 h}}{\partial \mu^{A^{\prime}} \partial \mu^{B^{\prime}} \cdots \partial \mu^{D^{\prime}}} f_{2 h-2}(W) \\
& =\mathrm{i}^{2 h} \delta\left(p^{2}\right) \int \mathrm{d}^{2} \mu \mathrm{e}^{-\mathrm{i}[\mu \tilde{\lambda}]} \tilde{\lambda}_{A^{\prime}} \tilde{\lambda}_{B^{\prime}} \cdots \tilde{\lambda}_{D^{\prime}} f_{2 h-2}(W),
\end{aligned}
$$

Both of these are captured by the simple half Fourier transforms

$$
\begin{aligned}
& \Phi_{2 h}(\lambda, \tilde{\lambda})=\int \mathrm{d}^{2} \mu \mathrm{e}^{-\mathrm{i}[\mu \tilde{\lambda}]} f_{2 h-2}(W) \\
& f_{2 h-2}(W)=\frac{1}{(2 \pi)^{2}} \int \mathrm{d}^{2} \tilde{\lambda} \mathrm{e}^{\mathrm{i}[\mu \tilde{\lambda}]} \Phi_{2 h}(\lambda, \tilde{\lambda})
\end{aligned} .
$$

It is easy to see that (B.21) implies that $f$ has homogeneity $2 h-2$ under $W_{\alpha} \rightarrow r W_{\alpha}$. This agrees with the well-known fact that massless fields of helicity $h$ on space-time or momentum space correspond to homogeneous functions on dual twistor space of weight $2 h-2$.

\section{B.3 Supersymmetry}

The Penrose transform naturally extends to supersymmetric theories by adjoining $\mathcal{N}$ anticommuting variables to the non-projective twistor space. Supertwistor space is then the projectivisation of $\mathbb{R}^{4 \mid \mathcal{N}}$ (or $\mathbb{C}^{4 \mid \mathcal{N}}$, in the complex case [93]). The space of $\mathbb{R P}^{1}$ s inside projective supertwistor space is the (conformal compactification of) anti-chiral split signature superspace with co-ordinates $\left(x_{-}^{A A^{\prime}}, \tilde{\theta}^{a A^{\prime}}\right)$, while the space of $\mathbb{R P}^{1} \mathrm{~s}$ inside dual projective supertwistor space is chiral superspace ${ }^{30}$ with co-ordinates $\left(x_{+}^{A A^{\prime}}, \theta_{a}^{A}\right)$. The incidence relation

\footnotetext{
${ }^{29}$ The factor of $\mathrm{i}^{2 h}$ is included for later convenience.

${ }^{30}$ Chiral superspace can also be realised as the space of $\mathbb{R} \mathbb{P}^{1 \mid \mathcal{N}_{\mathrm{S}}}$ inside supertwistor space, and similarly anti-chiral superspace is also the space of $\mathbb{R} \mathbb{P}^{1 \mid \mathcal{N}_{\mathrm{S}}}$ in dual supertwistor space, but we will not use this fact here.
} 
on dual supertwistor space is

$$
\mu^{A^{\prime}}=-x_{+}^{A A^{\prime}} \lambda_{A}, \quad \chi_{a}=\theta_{a}^{A} \lambda_{A} .
$$

Supertwistor space and its dual carry a natural action of the (4:1 cover of the) spacetime superconformal group $\operatorname{SL}(4 \mid \mathcal{N} ; \mathbb{R})$. Concentrating on the dual twistor space, this action is generated by the vector fields

$$
\left(W_{\alpha} \frac{\partial}{\partial W_{\beta}}-\frac{1}{4-\mathcal{N}} \delta_{\alpha}^{\beta} \Upsilon\right), \quad W_{\alpha} \frac{\partial}{\partial \chi_{b}}, \quad \chi_{a} \frac{\partial}{\partial W_{\beta}}, \quad\left(\chi_{a} \frac{\partial}{\partial \chi_{b}}-\frac{1}{4-\mathcal{N}} \delta_{a}^{b} \Upsilon\right)
$$

where $\left(W_{\alpha}, \chi_{a}\right)$ are homogeneous co-ordinates on the superspace and

$$
\Upsilon:=W_{\gamma} \frac{\partial}{\partial W_{\gamma}}+\chi_{c} \frac{\partial}{\partial \chi_{c}}
$$

is the Euler homogeneity operator. ${ }^{31}$

\section{B.3.1 Superfields in twistor space and on-shell momentum space}

When $\mathcal{N}=4$, we can construct a twistor supermultiplet representing an on-shell SYM multiplet by taking a (Lie algebra-valued) function $A(W, \chi)$ of the supertwistors, homogeneous of degree 0 as in [93]. Its component expansion is

$$
A(W, \chi)=a(W)+\chi_{a} \psi^{a}(W)+\cdots+\frac{1}{4 !} \epsilon^{a b c d} \chi_{a} \chi_{b} \chi_{c} \chi_{d} g(W)
$$

where the component fields $a, \psi^{a}, \ldots, g$ have homogeneities $0,-1, \ldots,-4$ corresponding to on-shell space-time fields of helicities $1, \frac{1}{2}, \ldots,-1$ and transform in the appropriate representation of the $\mathrm{SL}(4 ; \mathbb{R})$ R-symmetry group. We want to understand the relation between this representation and the on-shell momentum supermultiplet

$$
\Phi(\lambda, \tilde{\lambda}, \eta)=G^{+}(\lambda, \tilde{\lambda})+\eta^{a} \Gamma_{a}(\lambda, \tilde{\lambda})+\cdots+\frac{1}{4 !} \epsilon_{a b c d} \eta^{a} \eta^{b} \eta^{c} \eta^{d} G^{-}(\lambda, \tilde{\lambda})
$$

used by e.g. [14, 33-35, 48] in the supersymmetric BCFW Yang-Mills recursion relations.

Taking the X-ray transform of the complete supermultiplet (B.28) gives a chiral superfield $^{32}$

$$
\phi_{A^{\prime} B^{\prime}}(x, \theta)=\int\langle\lambda \mathrm{d} \lambda\rangle \frac{\partial^{2}}{\partial \mu^{A^{\prime}} \partial \mu^{B^{\prime}}} A\left(\lambda_{A},-x^{A A^{\prime}} \lambda_{A}, \theta_{a}^{A} \lambda_{A}\right) .
$$

$\phi_{A^{\prime} B^{\prime}}(x, \theta)$ is independent of the anti-chiral $\tilde{\theta}_{\mathrm{s}}$ and can be defined without auxiliary fields precisely because the multiplet is on-shell (see e.g. [94] for a full discussion). By differentiating under the integral sign, we see that

$$
\nabla^{A A^{\prime}} \phi_{A^{\prime} B^{\prime}}=0 \quad \text { and } \quad \nabla^{C C^{\prime}} \frac{\partial}{\partial \theta_{a}^{C}} \phi_{A^{\prime} B^{\prime}}=0 .
$$

\footnotetext{
${ }^{31}$ For $\mathcal{N}=4, \mathrm{SL}(4 \mid 4)$ needs to be defined slightly differently as being generated by linear transformations of $\mathbb{R}^{4 \mid 4}$ that have vanishing supertrace — we can no longer use $\Upsilon$ to remove the trace because $\operatorname{str}(\mathbb{I})=0$ when $\mathcal{N}=4$. Instead, $\Upsilon$ may either be considered as a bona fide generator of the group itself, or else may be omitted, in which case one is dealing with PSL(4|4).

${ }^{32}$ We henceforth drop the subscript on the chiral co-ordinate $x_{+}$.
} 
We now take the super-Fourier transform of $\phi_{A^{\prime} B^{\prime}}$, i.e.

$$
\tilde{\phi}_{A^{\prime} B^{\prime}}(p, \xi)=\int \mathrm{d}^{4 \mid 8} x \exp \left(-\mathrm{i} x^{C C^{\prime}} p_{C C^{\prime}}-\mathrm{i} \theta_{a}^{C} \xi_{C}^{a}\right) \phi_{A^{\prime} B^{\prime}}(x, \theta),
$$

where $\mathrm{d}^{4 \mid 8} x=\mathrm{d}^{4} x \mathrm{~d}^{8} \theta$ is the usual chiral superspace measure and $\xi_{A}^{a}$ is conjugate to $\theta_{a}^{A}$. The superfield equations (B.31) imply that $\tilde{\phi}_{A^{\prime} B^{\prime}}(p, \xi)$ obeys

$$
p^{A A^{\prime}} \tilde{\phi}_{A^{\prime} B^{\prime}}(p, \xi)=0 \quad \text { and } \quad p^{C C^{\prime}} \xi_{C}^{a} \tilde{\phi}_{A^{\prime} B^{\prime}}(p, \xi)=0 .
$$

As before, the first of these implies that

$$
\tilde{\phi}_{A^{\prime} B^{\prime}}(p, \xi)=\delta\left(p^{2}\right) \tilde{\lambda}_{A^{\prime}} \tilde{\lambda}_{B^{\prime}} \Phi(\lambda, \tilde{\lambda}, \xi)
$$

with $p_{A A^{\prime}}=\lambda_{A} \tilde{\lambda}_{A^{\prime}}$ on-shell. The second equation in (B.33) means that $\Phi(\lambda, \tilde{\lambda}, \xi)$ vanishes when multiplied by $\lambda^{A} \xi_{A}^{a}$ for any choice of the R-symmetry index. Thus the super-Fourier transform is a multiple of

$$
\delta^{(0 \mid 4)}\left(\xi_{A}^{a} \lambda^{A}\right):=\frac{1}{4 !} \epsilon_{a b c d} \xi_{A}^{a} \xi_{B}^{b} \xi_{C}^{c} \xi_{D}^{d} \lambda^{A} \lambda^{B} \lambda^{C} \lambda^{D} .
$$

and the factor $\delta\left(p^{2}\right) \delta^{(0 \mid 4)}\left(\xi_{A}^{a} \lambda^{A}\right)$ restricts the support of the super Fourier transform to the 'super light-cone' $p^{2}=0=\xi_{A}^{a} \lambda^{A}$ in momentum superspace. On restriction to this super light-cone, $\xi_{A}^{a} \lambda^{A} \delta^{(0 \mid 4)}\left(\xi_{B}^{b} \lambda^{B}\right)=0$, so we have

$$
\xi_{A}^{a} \delta^{(0 \mid 4)}\left(\xi_{B}^{b} \lambda^{B}\right)=: \eta^{a} \lambda_{A} \delta^{(0 \mid 4)}\left(\xi_{B}^{b} \lambda^{B}\right)
$$

for some odd co-ordinates $\eta^{a}$ with opposite weight to $\lambda_{A}$ (the same weight as $\tilde{\lambda}_{A^{\prime}}$ ). Thus we co-ordinatise the momentum space super-light-cone by $\left(\lambda_{A}, \tilde{\lambda}_{A^{\prime}}, \eta^{a}\right)$ defined up to the scaling

$$
\left(\lambda_{A}, \tilde{\lambda}_{A^{\prime}}, \eta^{a}\right) \sim\left(r \lambda_{A}, r^{-1} \tilde{\lambda}_{A^{\prime}}, r^{-1} \eta^{a}\right), \quad r \in \mathbb{R} .
$$

On this super light-cone $\left(p_{A A^{\prime}}, \xi_{A}^{a}\right)=\left(\lambda_{A} \tilde{\lambda}_{A^{\prime}}, \lambda_{A} \eta^{a}\right)$ and a wavefunction may be written as

$$
\tilde{\phi}_{A^{\prime} B^{\prime}}(p, \xi)=\delta\left(p^{2}\right) \delta^{(0 \mid 4)}\left(\xi_{A}^{a} \lambda^{A}\right) \tilde{\lambda}_{A^{\prime}} \tilde{\lambda}_{B^{\prime}} \Phi_{-2}(\lambda, \tilde{\lambda}, \eta) .
$$

Combining equations (B.30) \& (B.32), and following the same argument as before, but now performing the integral over both the $\chi^{A^{\prime}}$ of (B.8) and the $\zeta^{a}$ of $\theta_{a}^{A}=\left(\nu_{a} \alpha^{A}+\zeta_{a} \lambda^{\prime A}\right) /\langle\alpha \lambda\rangle$ yields the extra delta function $\delta^{(0 \mid 4)}\left(\xi_{A}^{a} \lambda^{A}\right)$. Omitting the details, we obtain the formula

$$
\Phi_{-2}(\lambda, \tilde{\lambda}, \eta)=\frac{1}{(2 \pi)^{2}} \int \mathrm{d}^{2 \mid 4} \mu \mathrm{e}^{-\mathrm{i} \llbracket \mu \tilde{\lambda} \rrbracket} A(W, \chi)
$$

where we use the supersymmetric notation

$$
\llbracket \mu \tilde{\lambda} \rrbracket:=[\mu \tilde{\lambda}]+\chi_{a} \eta^{a} .
$$

Thus the on-shell momentum space SYM multiplet (B.29) is simply the half Fourier transform (taken over both $\mu^{A^{\prime}}$ and $\chi_{a}$ ) of the twistor supermultiplet. 
The same arguments applies for all $\mathcal{N}$, and in particular for an $\mathcal{N}=8$ supergravity multiplet: According to the X-ray or Penrose transform, a linearized graviton of helicity +2 may be represented on dual twistor space by a twistor function $h_{2}(W)$ of homogeneity +2 , while a graviton of helicity -2 is represented by a twistor function $h_{-6}(W)$ of weight -6 . For $\mathcal{N}=8$ supertwistor space, both graviton helicities are contained in the single twistor supermultiplet

$$
H(W, \chi)=h_{2}(W)+\chi_{a} \gamma^{a}(W)+\cdots+(\chi)^{8} h_{-6}(W) .
$$

The above extends straightforwardly to a proof that this multiplet corresponds to an onshell $\mathcal{N}=8$ supergravity multiplet in momentum space via the half Fourier transform

$$
\Phi_{-4}(\lambda, \tilde{\lambda}, \eta)=\frac{1}{(2 \pi)^{2}} \int \mathrm{d}^{2 \mid 8} \mu \mathrm{e}^{-\mathrm{i} \llbracket \mu \tilde{\lambda} \rrbracket} H(W, \chi)
$$

and again $\Phi_{-4}(\lambda, \tilde{\lambda}, \eta)$ is the supermultiplet used in the BCFW rules for $\mathcal{N}=8 \mathrm{SG}[48,53]$.

We finally remark that the half Fourier transform yields the supersymmetric substitutions

$$
\begin{aligned}
\tilde{\lambda}_{A^{\prime}} & \rightarrow \mathrm{i} \frac{\partial}{\partial \mu^{A^{\prime}}} \quad \frac{\partial}{\partial \tilde{\lambda}_{A^{\prime}}} \rightarrow-\mathrm{i} \mu^{A^{\prime}} \\
\eta^{a} & \rightarrow \mathrm{i} \frac{\partial}{\partial \chi_{a}}, \quad \frac{\partial}{\partial \eta^{a}} \rightarrow-\mathrm{i} \chi_{a}
\end{aligned}
$$

This relates the dual twistor space superconformal generators of (B.26) to

$$
\begin{aligned}
Q_{A a} & =\lambda_{A} \frac{\partial}{\partial \eta^{a}} \quad \tilde{Q}_{A^{\prime}}^{b}=\tilde{\lambda}_{A^{\prime}} \eta^{b} \\
S^{A b} & =\eta^{b} \frac{\partial}{\partial \lambda_{A}} \quad \tilde{S}_{a}^{A^{\prime}}=\frac{\partial^{2}}{\partial \eta^{a} \partial \tilde{\lambda}_{A^{\prime}}}
\end{aligned}
$$

together with the R-symmetry generator

$$
R_{b}^{a}=\eta^{a} \frac{\partial}{\partial \eta^{b}}-\frac{1}{\mathcal{N}} \delta^{a}{ }_{b} \eta^{c} \frac{\partial}{\partial \eta^{c}}
$$

on the momentum super light-cone.

\section{The half Fourier transform of seed amplitudes}

In this appendix we explicitly perform the half Fourier transform of the 3-particle MHV and $\overline{\mathrm{MHV}}$, and the $n$-particle MHV SYM amplitudes to (dual) twistor space.

\section{C.1 The 3-point MHV amplitude}

The three-particle MHV amplitude in on-shell momentum superspace is

$$
A_{\mathrm{MHV}}\left(p_{1}, p_{2}, p_{3}\right)=\frac{\delta^{(4 \mid 8)}\left(\sum_{i=1}^{3}|i\rangle \llbracket i \|\right)}{\langle 12\rangle\langle 23\rangle\langle 31\rangle}
$$


where again $\llbracket i \|=\left(\left[i \mid, \eta_{i}\right)\right.$. We will re-express the momentum $\delta$-functions as spinor $\delta$ functions to expedite the half Fourier transforms. For the three-point MHV amplitude we may assume $\langle 12\rangle \neq 0$ and so can expand unprimed spinors in the basis $\{|1\rangle,|2\rangle\}$. This gives

$$
\sum_{i=1}^{3}|i\rangle \llbracket i \|=|1\rangle\left(\llbracket 1\left\|+\frac{\langle 32\rangle}{\langle 12\rangle} \llbracket 3\right\|\right)+|2\rangle\left(\llbracket 2\left\|+\frac{\langle 13\rangle}{\langle 12\rangle} \llbracket 3\right\|\right)
$$

and since $|1\rangle$ and $|2\rangle$ are linearly independent, the $\delta$-function becomes

$$
\delta^{(4 \mid 8)}\left(\sum_{i=1}^{3}|i\rangle \llbracket i \|\right)=\langle 12\rangle^{2} \delta^{(2 \mid 4)}\left(\llbracket 1\left\|+\frac{\langle 32\rangle}{\langle 12\rangle} \llbracket 3\right\|\right) \delta^{(2 \mid 4)}\left(\llbracket 2\left\|+\frac{\langle 13\rangle}{\langle 12\rangle} \llbracket 3\right\|\right)
$$

The half Fourier transform of states 1 and 2 can be done straightforwardly using these $\delta$-functions, yielding

$$
\mathcal{A}_{\mathrm{MHV}}\left(\mathrm{W}_{1}, \mathrm{~W}_{2}, \mathrm{~W}_{3}\right)=\frac{\delta^{(2 \mid 4)}\left(\mu_{1}\langle 23\rangle+\mu_{2}\langle 31\rangle+\mu_{3}\langle 12\rangle\right)}{\langle 12\rangle\langle 23\rangle\langle 31\rangle}
$$

as in equation (3.2). This has homogeneity degree zero in each of the three supertwistors, as required. It has support precisely where $\mathrm{W}_{1}, \mathrm{~W}_{2}$ and $\mathrm{W}_{3}$ are collinear and is appropriately antisymmetric under permutations of $1,2,3$.

We can elucidate the behaviour of (C.4) under conformal transformation by relating it to the superconformally invariant $\tilde{\delta}$-function

$$
\tilde{\delta}^{(2 \mid 4)}\left(\mathrm{W}_{1} ; \mathrm{W}_{2}, \mathrm{~W}_{3}\right)=\int \frac{\mathrm{d} s}{s} \frac{\mathrm{d} t}{t} \delta^{(4 \mid 4)}\left(\mathrm{W}_{1}-s \mathrm{~W}_{2}-t \mathrm{~W}_{3}\right)
$$

Notice that this $\tilde{\delta}$-function is manifestly symmetric under the exchange $2 \leftrightarrow 3$. By using the $|\lambda\rangle$ spinor components to perform the integrals we obtain

$$
\int \frac{\mathrm{d} s}{s} \frac{\mathrm{d} t}{t} \delta^{(2)}\left(\lambda_{1}-s \lambda_{2}-t \lambda_{3}\right)=\frac{|\langle 23\rangle|}{\langle 12\rangle\langle 31\rangle} ; \quad s=\frac{\langle 31\rangle}{\langle 21\rangle}, \quad t=\frac{\langle 21\rangle}{\langle 32\rangle},
$$

where the modulus sign arises from a Jacobian in the delta functions, generalising the standard scaling $\delta(a x)=|a|^{-1} \delta(x)$. Combining this with the $\| \mu \rrbracket$ components of $\tilde{\delta}^{(2 \mid 4)}\left(\mathrm{W}_{1}, \mathrm{~W}_{2}, \mathrm{~W}_{3}\right)$ yields

$$
\tilde{\delta}^{(2 \mid 4)}\left(\mathrm{W}_{1}, \mathrm{~W}_{2}, \mathrm{~W}_{3}\right)=\operatorname{sgn}(\langle 23\rangle) \frac{\delta^{(2 \mid 4)}\left(\mu_{1}\langle 23\rangle+\mu_{2}\langle 31\rangle+\mu_{3}\langle 12\rangle\right)}{\langle 12\rangle\langle 23\rangle\langle 31\rangle}
$$

Thus we see that

$$
\mathcal{A}_{\mathrm{MHV}}\left(\mathrm{W}_{1}, \mathrm{~W}_{2}, \mathrm{~W}_{3}\right)=\operatorname{sgn}\left(\left\langle\mathrm{W}_{2} \mathrm{~W}_{3}\right\rangle\right) \tilde{\delta}^{(2 \mid 4)}\left(\mathrm{W}_{1} ; \mathrm{W}_{2}, \mathrm{~W}_{3}\right)
$$

as in equation (3.28). 


\section{C.2 The 3-point $\overline{\mathrm{MHV}}$ amplitude}

The three-particle $\overline{\mathrm{MHV}}$ amplitude for $\mathcal{N}=4 \mathrm{SYM}$ in on-shell momentum superspace is given by [34]

$$
A_{\overline{\mathrm{MHV}}}\left(p_{1}, p_{2}, p_{3}\right)=\frac{\delta^{(4)}\left(p_{1}+p_{2}+p_{3}\right) \delta^{(4)}\left(\eta_{1}[23]+\eta_{2}[31]+\eta_{3}[12]\right)}{[12][23][31]} .
$$

Momentum conservation here implies proportionality of the unprimed spinors, so to construct a basis for this space we must introduce an arbitrary independent spinor $|\alpha\rangle$ with $\langle 1 \alpha\rangle \neq 0$. We then express $|2\rangle$ and $|3\rangle$, say, in the $\{|1\rangle,|\alpha\rangle\}$ basis, leading to

$$
\begin{aligned}
\delta^{(4)}\left(p_{1}+p_{2}+p_{3}\right) & \left.\left.\left.\left.\left.=\frac{1}{\langle 1 \alpha\rangle^{2}} \delta^{(2)}(\mid 1]+\mid 2\right] \frac{\langle 2 \alpha\rangle}{\langle 1 \alpha\rangle}+\mid 3\right] \frac{\langle 3 \alpha\rangle}{\langle 1 \alpha\rangle}\right) \delta^{(2)}(\mid 2] \frac{\langle 21\rangle}{\langle\alpha 1\rangle}+\mid 3\right] \frac{\langle 31\rangle}{\langle\alpha 1\rangle}\right) \\
& \left.\left.\left.=\frac{1}{|[23]|} \delta^{(2)}(\mid 1]+\mid 2\right] \frac{\langle 2 \alpha\rangle}{\langle 1 \alpha\rangle}+\mid 3\right] \frac{\langle 3 \alpha\rangle}{\langle 1 \alpha\rangle}\right) \delta(\langle 12\rangle) \delta(\langle 13\rangle),
\end{aligned}
$$

The second line follows from the fact that $\mid 2]$ and $\mid 3]$ are linearly independent in the $\overline{\mathrm{MHV}}$ amplitude and the pre-factors $1 /\langle 1 \alpha\rangle^{2}$ in the first line and $1 /|[23]|$ in the second come from Jacobians. The modulus sign in the second Jacobian again arises from the scaling relation $\delta(a x)=|a|^{-1} \delta(x)$ for the standard 1-dimensional $\delta$-function. On the support of the $\delta^{(2)}$-functions for $\left.\mid 1\right]$, the remaining unprimed spinor factors in (C.9) become

$$
\frac{\delta^{(4)}\left(\eta_{1}[23]+\eta_{2}[31]+\eta_{3}[12]\right)}{[12][23][31]}=\frac{[23]\langle 1 \alpha\rangle^{2}}{\langle 2 \alpha\rangle\langle 3 \alpha\rangle} \delta^{(4)}\left(\eta_{1}+\eta_{2} \frac{\langle 2 \alpha\rangle}{\langle 1 \alpha\rangle}+\eta_{3} \frac{\langle 3 \alpha\rangle}{\langle 1 \alpha\rangle}\right) .
$$

Notice that the factor of [23] in the numerator here cancels the overall Jacobian in (C.10) only up to its sign.

The twistor $\overline{\mathrm{MHV}}$ amplitude is the Fourier transform

$$
\begin{aligned}
\mathcal{A}_{\overline{\mathrm{MHV}}}\left(\mathrm{W}_{1}, \mathrm{~W}_{2}, \mathrm{~W}_{3}\right)=\delta(\langle 12\rangle) \delta(\langle 13\rangle) \frac{\langle 1 \alpha\rangle^{2}}{\langle 2 \alpha\rangle\langle 3 \alpha\rangle} \\
\quad \times \int \prod_{j=1}^{3} \mathrm{~d}^{2 \mid 4} \tilde{\lambda}_{j} \mathrm{e}^{\mathrm{i} \llbracket \mu_{j} \tilde{\lambda}_{j} \rrbracket} \operatorname{sgn}([23]) \delta^{(2 \mid 4)}\left(\llbracket 1\|+\llbracket 2\| \frac{\langle 2 \alpha\rangle}{\langle 1 \alpha\rangle}+\llbracket 3 \| \frac{\langle 3 \alpha\rangle}{\langle 1 \alpha\rangle}\right)
\end{aligned}
$$

The $\mathrm{d}^{2 \mid 4} \tilde{\lambda}_{1}$ integral may be done immediately using the delta functions, yielding

$$
\begin{aligned}
\int & \prod_{j=2,3}\left\{\mathrm{~d}^{2 \mid 4} \tilde{\lambda}_{j} \mathrm{e}^{\mathrm{i} \llbracket \mu_{j} \tilde{\lambda}_{j} \rrbracket-\mathrm{i} \frac{\langle j \alpha\rangle}{\langle 1 \alpha\rangle} \llbracket \mu_{1} \tilde{\lambda}_{j} \rrbracket} \delta(\langle 1 j\rangle) \frac{\langle 1 \alpha\rangle}{\langle j \alpha\rangle}\right\} \operatorname{sgn}[23] \\
& =\int \prod_{j=2,3}\left\{\mathrm{~d}^{2 \mid 4} \mu_{j}^{\prime} \mathrm{d}^{2 \mid 4} \tilde{\lambda}_{j} \mathrm{e}^{\mathrm{i} \llbracket \mu_{i} \tilde{\lambda}_{i} \rrbracket-\mathrm{i} \llbracket \mu_{i}^{\prime} \tilde{\lambda}_{i} \rrbracket} \delta^{(2 \mid 4)}\left(\mu_{1}-\mu_{j}^{\prime} \frac{\langle 1 \alpha\rangle}{\langle j \alpha\rangle}\right) \frac{\langle j \alpha\rangle}{\langle 1 \alpha\rangle} \delta(\langle 1 j\rangle)\right\} \operatorname{sgn}[23] .
\end{aligned}
$$

In the second line here, we have introduced integrals over dummy variables $\mu_{j}^{\prime}$ together with weighted $\delta$ functions which re-enforce $\mu_{j}^{\prime}=\frac{\langle j \alpha\rangle}{\langle 1 \alpha\rangle} \mu_{1}$. The virtue of this step is that, since we can also write

$$
\frac{\langle j \alpha\rangle}{\langle 1 \alpha\rangle} \delta(\langle 1 j\rangle)=\int_{-\infty}^{\infty} \frac{\mathrm{d} t}{t} \delta^{(2)}(|1\rangle-t|j\rangle)
$$


the $\delta$-functions combine into our superconformally invariant, but tilded $\delta$-functions

$$
\delta^{(2 \mid 4)}\left(\mu_{1}-\mu_{j}^{\prime} \frac{\langle 1 \alpha\rangle}{\langle j \alpha\rangle}\right) \frac{\langle j \alpha\rangle}{\langle 1 \alpha\rangle} \delta(\langle 1 j\rangle)=\int \frac{\mathrm{d} t}{t} \delta^{(4 \mid 4)}\left(\mathrm{W}_{1}-t \mathrm{~W}_{j}^{\prime}\right)=: \tilde{\delta}^{(3 \mid 4)}\left(\mathrm{W}_{1}, \mathrm{~W}_{j}^{\prime}\right),
$$

where $\mathrm{W}_{j}^{\prime}$ is the supertwistor $\left(\lambda_{j}, \mu_{j}^{\prime}, \chi_{j}^{\prime}\right)$ for $j=2,3$.

As in the BCFW recursion relations, we now replace the factor sgn[23] by the formal operator

$$
\operatorname{sgn}[23]=\operatorname{sgn}\left[\frac{\partial}{\partial \mu_{2}^{\prime}} \frac{\partial}{\partial \mu_{3}^{\prime}}\right]=\operatorname{sgn}\left[\frac{\partial}{\partial \mathrm{W}_{2}^{\prime}} \frac{\partial}{\partial \mathrm{W}_{3}^{\prime}}\right]
$$

acting inside the $\mathrm{d}^{2 \mid 4} \tilde{\lambda}_{j} \mathrm{~d}^{2 \mid 4} \mu_{j}^{\prime}$ integrals. Performing these integrals then simply replaces $\mathrm{W}_{j}^{\prime}$ by $\mathrm{W}_{j}$. Overall, the dual twistor form of the three-point $\overline{\mathrm{MHV}}$ super-amplitude in $\mathcal{N}=4 \mathrm{SYM}$ is

$$
\mathcal{A}_{\overline{\mathrm{MHV}}}\left(\mathrm{W}_{1}, \mathrm{~W}_{2}, \mathrm{~W}_{3}\right)=\operatorname{sgn}\left(\left[\frac{\partial}{\partial \mathrm{W}_{2}} \frac{\partial}{\partial \mathrm{W}_{3}}\right]\right) \tilde{\delta}^{(3 \mid 4)}\left(\mathrm{W}_{1}, \mathrm{~W}_{2}\right) \tilde{\delta}^{(3 \mid 4)}\left(\mathrm{W}_{1}, \mathrm{~W}_{3}\right)
$$

as in equation (3.32). In fact, it is easy to show that $\mathcal{A}_{\overline{\mathrm{MHV}}}\left(\mathrm{W}_{1}, \mathrm{~W}_{2}, \mathrm{~W}_{3}\right)$ is given by the explicit formula

$$
\begin{aligned}
& \mathcal{A}_{\overline{\mathrm{MHV}}}\left(\mathrm{W}_{1}, \mathrm{~W}_{2}, \mathrm{~W}_{3}\right)=\frac{\lambda_{1}^{2}}{\lambda_{2} \lambda_{3}} \delta(\langle 12\rangle) \delta(\langle 13\rangle) \times \\
& \times \delta^{\prime}\left(\left(\mu_{2}^{A^{\prime}}-\frac{\lambda_{2}}{\lambda_{1}} \mu_{1}^{A^{\prime}}\right)\left(\mu_{3 A^{\prime}}-\frac{\lambda_{3}}{\lambda_{1}} \mu_{1 A^{\prime}}\right)\right) \delta^{(0 \mid 4)}\left(\chi_{2}-\frac{\lambda_{2}}{\lambda_{1}} \chi_{1}\right) \delta^{(0 \mid 4)}\left(\chi_{3}-\frac{\lambda_{3}}{\lambda_{1}} \chi_{1}\right),
\end{aligned}
$$

where the ratios $\lambda_{2} / \lambda_{1}$ and $\lambda_{3} / \lambda_{1}$ are meaningful on the support of $\delta(\langle 12\rangle) \delta(\langle 13\rangle)$.

\section{C.3 The n-point MHV amplitude}

The $n$-point Parke-Taylor super-amplitude is

$$
A_{\mathrm{MHV}}\left(p_{1}, \ldots, p_{n}\right)=\frac{\delta^{(4 \mid 8)}\left(\sum_{i=1}^{n}|i\rangle \llbracket i \|\right)}{\langle 12\rangle\langle 23\rangle \cdots\langle n 1\rangle} .
$$

To transform (C.19) to dual twistor space, we use a straightforward extension of (C.3) for the momentum $\delta$-function, using the unprimed spin basis $\{|1\rangle,|2\rangle\}$ to rewrite it as

$$
\delta^{(4 \mid 8)}\left(\sum_{i=1}^{n}|i\rangle \llbracket i \|\right)=\langle 12\rangle^{2} \delta^{(2 \mid 4)}\left(\llbracket 1\left\|+\sum_{i=3}^{n} \frac{\langle i 2\rangle}{\langle 12\rangle} \llbracket i\right\|\right) \delta^{(2 \mid 4)}\left(\llbracket 2\left\|+\sum_{i=3}^{n} \frac{\langle i 1\rangle}{\langle 21\rangle} \llbracket i\right\|\right) .
$$

Insert this into the the half Fourier transform and immediately performing the $\llbracket 1 \|$ and $\llbracket 2 \|$ integrals one obtains

$$
\begin{aligned}
\mathcal{A}_{\mathrm{MHV}}\left(\mathrm{W}_{1}, \ldots, \mathrm{W}_{n}\right) & =\frac{\langle 12\rangle}{\langle n 1\rangle} \prod_{i=3}^{n} \frac{1}{\langle i-1 i\rangle} \delta^{(2 \mid 4)}\left(\mu_{i}+\frac{\langle i 1\rangle}{\langle 12\rangle} \mu_{2}-\frac{\langle i 2\rangle}{\langle 12\rangle} \mu_{1}\right) \\
& =\frac{\langle 12\rangle}{\langle n 1\rangle} \prod_{i=3}^{n} \frac{1}{\langle i-1 i\rangle\langle 12\rangle^{2}} \delta^{(2 \mid 4)}\left(\mu_{1}\langle 2 i\rangle+\mu_{2}\langle i 1\rangle+\mu_{i}\langle 12\rangle\right)
\end{aligned}
$$


Using the identity

$$
\begin{aligned}
& \delta^{(2 \mid 4)}\left(\mu_{1}\langle 2 i\rangle+\mu_{2}\langle i 1\rangle+\mu_{i}\langle 12\rangle\right) \delta^{(2 \mid 4)}\left(\mu_{1}\langle 2 j\rangle+\mu_{2}\langle j 1\rangle+\mu_{j}\langle 12\rangle\right) \\
& =\frac{\langle 12\rangle^{2}}{\langle 1 i\rangle^{2}} \delta^{(2 \mid 4)}\left(\mu_{1}\langle 2 i\rangle+\mu_{2}\langle i 1\rangle+\mu_{i}\langle 12\rangle\right) \delta^{(2 \mid 4)}\left(\mu_{1}\langle i j\rangle+\mu_{i}\langle j 1\rangle+\mu_{j}\langle 1 i\rangle\right),
\end{aligned}
$$

one can show

$$
\mathcal{A}_{\mathrm{MHV}}\left(\mathrm{W}_{1}, \ldots, \mathrm{W}_{n}\right)=(-1)^{n-3} \prod_{i=3}^{n} \mathcal{A}_{\mathrm{MHV}}\left(\mathrm{W}_{1}, \mathrm{~W}_{i-1}, \mathrm{~W}_{i}\right)
$$

as in equation (3.29).

Open Access. This article is distributed under the terms of the Creative Commons Attribution Noncommercial License which permits any noncommercial use, distribution, and reproduction in any medium, provided the original author(s) and source are credited.

\section{References}

[1] E. Witten, Perturbative gauge theory as a string theory in twistor space, Commun. Math. Phys. 252 (2004) 189 [hep-th/0312171] [SPIRES].

[2] R. Roiban, M. Spradlin and A. Volovich, On the tree-level S-matrix of Yang-Mills theory, Phys. Rev. D 70 (2004) 026009 [hep-th/0403190] [SPIRES].

[3] F. Cachazo, P. Svrček and E. Witten, MHV vertices and tree amplitudes in gauge theory, JHEP 09 (2004) 006 [hep-th/0403047] [SPIRES].

[4] R. Britto, F. Cachazo and B. Feng, New recursion relations for tree amplitudes of gluons, Nucl. Phys. B 715 (2005) 499 [hep-th/0412308] [SPIRES].

[5] R. Britto, F. Cachazo, B. Feng and E. Witten, Direct proof of tree-level recursion relation in Yang-Mills theory, Phys. Rev. Lett. 94 (2005) 181602 [hep-th/0501052] [SPIRES].

[6] R. Britto, F. Cachazo and B. Feng, Generalized unitarity and one-loop amplitudes in $N=4$ super-Yang-Mills, Nucl. Phys. B 725 (2005) 275 [hep-th/0412103] [SPIRES].

[7] Z. Bern, L.J. Dixon and V.A. Smirnov, Iteration of planar amplitudes in maximally supersymmetric Yang-Mills theory at three loops and beyond, Phys. Rev. D 72 (2005) 085001 [hep-th/0505205] [SPIRES].

[8] Z. Bern, M. Czakon, L.J. Dixon, D.A. Kosower and V.A. Smirnov, The four-loop planar amplitude and cusp anomalous dimension in maximally supersymmetric Yang-Mills theory, Phys. Rev. D 75 (2007) 085010 [hep-th/0610248] [SPIRES].

[9] Z. Bern, J.J.M. Carrasco, H. Johansson and D.A. Kosower, Maximally supersymmetric planar Yang-Mills amplitudes at five loops, Phys. Rev. D 76 (2007) 125020 [arXiv:0705.1864] [SPIRES].

[10] E.I. Buchbinder and F. Cachazo, Two-loop amplitudes of gluons and octa-cuts in $N=4$ super Yang-Mills, JHEP 11 (2005) 036 [hep-th/0506126] [SPIRES].

[11] F. Cachazo and D. Skinner, On the structure of scattering amplitudes in $N=4$ super Yang-Mills and $N=8$ supergravity, arXiv:0801.4574 [SPIRES]. 
[12] F. Cachazo, Sharpening the leading singularity, arXiv:0803.1988 [SPIRES].

[13] F. Cachazo, M. Spradlin and A. Volovich, Leading singularities of the two-loop six-particle MHV amplitude, Phys. Rev. D 78 (2008) 105022 [arXiv:0805.4832] [SPIRES].

[14] J.M. Drummond, J. Henn, G.P. Korchemsky and E. Sokatchev, Generalized unitarity for $N=4$ super-amplitudes, arXiv:0808.0491 [SPIRES].

[15] F. Cachazo, P. Svrček and E. Witten, Twistor space structure of one-loop amplitudes in gauge theory, JHEP 10 (2004) 074 [hep-th/0406177] [SPIRES].

[16] F. Cachazo, P. Svrček and E. Witten, Gauge theory amplitudes in twistor space and holomorphic anomaly, JHEP 10 (2004) 077 [hep-th/0409245] [SPIRES].

[17] I. Bena, Z. Bern and D.A. Kosower, Twistor-space recursive formulation of gauge theory amplitudes, Phys. Rev. D 71 (2005) 045008 [hep-th/0406133] [SPIRES].

[18] I. Bena, Z. Bern, D.A. Kosower and R. Roiban, Loops in twistor space, Phys. Rev. D 71 (2005) 106010 [hep-th/0410054] [SPIRES].

[19] J. Bedford, A. Brandhuber, B.J. Spence and G. Travaglini, A twistor approach to one-loop amplitudes in $N=1$ supersymmetric Yang-Mills theory, Nucl. Phys. B 706 (2005) 100 [hep-th/0410280] [SPIRES].

[20] R. Britto, F. Cachazo and B. Feng, Coplanarity in twistor space of $N=4$ next-to- $M H V$ one-loop amplitude coefficients, Phys. Lett. B 611 (2005) 167 [hep-th/0411107] [SPIRES].

[21] S.J. Bidder, N.E.J. Bjerrum-Bohr, D.C. Dunbar and W.B. Perkins, Twistor space structure of the box coefficients of $N=1$ one-loop amplitudes, Phys. Lett. B 608 (2005) 151 [hep-th/0412023] [SPIRES].

[22] E.T. Newman, Heaven and its properties, Gen. Rel. Grav. 7 (1976) 107 [SPIRES].

[23] R. Penrose, Non-linear gravitons and curved twistor theory, Gen. Rel. Grav. 7 (1976) 31 [SPIRES].

[24] R.S. Ward, On self-dual gauge fields, Phys. Lett. A 61 (1977) 81 [SPIRES].

[25] L.J. Mason and N.M.J. Woodhouse, Integrability, self-duality and twistor theory, London Mathematical Society Monographs, new series 15, Oxford University Press, Oxford U.K. (1996) [SPIRES].

[26] L.J. Mason, Twistor actions for non-self-dual fields: a derivation of twistor-string theory, JHEP 10 (2005) 009 [hep-th/0507269] [SPIRES].

[27] R. Boels, L. Mason and D. Skinner, Supersymmetric gauge theories in twistor space, JHEP 02 (2007) 014 [hep-th/0604040] [SPIRES].

[28] L.J. Mason and M. Wolf, A twistor action for $N=8$ self-dual supergravity, Commun. Math. Phys. 288 (2009) 97 [arXiv:0706.1941] [SPIRES].

[29] L. Mason and D. Skinner, Gravity, twistors and the MHV formalism, arXiv:0808.3907 [SPIRES].

[30] R. Boels, L. Mason and D. Skinner, From twistor actions to MHV diagrams, Phys. Lett. B 648 (2007) 90 [hep-th/0702035] [SPIRES].

[31] J.M. Drummond, J.M. Henn and J. Plefka, Yangian symmetry of scattering amplitudes in $N=4$ super Yang-Mills theory, JHEP 05 (2009) 046 [arXiv:0902.2987] [SPIRES]. 
[32] J.M. Drummond, J. Henn, G.P. Korchemsky and E. Sokatchev, Conformal Ward identities for Wilson loops and a test of the duality with gluon amplitudes, Nucl. Phys. B 826 (2010) 337 [arXiv:0712.1223] [SPIRES].

[33] J.M. Drummond, J. Henn, G.P. Korchemsky and E. Sokatchev, Dual superconformal symmetry of scattering amplitudes in $N=4$ super-Yang-Mills theory, Nucl. Phys. B 828 (2010) 317 [arXiv:0807.1095] [SPIRES].

[34] A. Brandhuber, P. Heslop and G. Travaglini, A note on dual superconformal symmetry of the $N=4$ super Yang-Mills S-matrix, Phys. Rev. D 78 (2008) 125005 [arXiv:0807.4097] [SPIRES].

[35] J.M. Drummond and J.M. Henn, All tree-level amplitudes in $N=4 S Y M$, JHEP 04 (2009) 018 [arXiv: 0808.2475] [SPIRES].

[36] Z. Bern et al., The two-loop six-gluon MHV amplitude in maximally supersymmetric Yang-Mills theory, Phys. Rev. D 78 (2008) 045007 [arXiv:0803.1465] [SPIRES].

[37] J.M. Drummond, J. Henn, G.P. Korchemsky and E. Sokatchev, Hexagon Wilson loop = six-gluon MHV amplitude, Nucl. Phys. B 815 (2009) 142 [arXiv:0803.1466] [SPIRES].

[38] R. Penrose, The central programme of twistor theory, Chaos Solitons Fractals 10 (1999) 581 [SPIRES].

[39] R. Penrose and M.A.H. MacCallum, Twistor theory: an approach to the quantization of fields and space-time, Phys. Rept. 6 (1972) 241 [SPIRES].

[40] A.P. Hodges and S. Huggett, Twistor diagrams, Surveys High Energ. Phys. 1 (1980) 333 [SPIRES].

[41] F. John, The ultra-hyperbolic differential equation with four independent variables, Duke Math. J. 4 (1938) 300 reprinted in 75 years of the Radon transform (Vienna Austria 1992), Conf. Proc. Lecture Notes Math. Phys. 4 (1994) 301, International Press, U.S.A. (1994).

[42] M.F. Atiyah, Geometry of Yang-Mills fields, Accademia Nazionale dei Lincei Scuola Normale Superiore, Lezione Fermiane, Pisa Italy (1979).

[43] A.P. Hodges, Twistor diagram recursion for all gauge-theoretic tree amplitudes, hep-th/0503060 [SPIRES].

[44] A.P. Hodges, Twistor diagrams for all tree amplitudes in gauge theory: a helicity-independent formalism, hep-th/0512336 [SPIRES].

[45] A.P. Hodges, Scattering amplitudes for eight gauge fields, hep-th/0603101 [SPIRES].

[46] N. Arkani-Hamed, F. Cachazo, C. Cheung and J. Kaplan, The S-matrix in twistor space, arXiv: 0903.2110 [SPIRES].

[47] P. Benincasa, C. Boucher-Veronneau and F. Cachazo, Taming tree amplitudes in general relativity, JHEP 11 (2007) 057 [hep-th/0702032] [SPIRES].

[48] N. Arkani-Hamed, F. Cachazo and J. Kaplan, What is the simplest quantum field theory?, arXiv:0808.1446 [SPIRES].

[49] R. Boels, A quantization of twistor Yang-Mills theory through the background field method, Phys. Rev. D 76 (2007) 105027 [hep-th/0703080] [SPIRES]. 
[50] A. Brandhuber, B.J. Spence and G. Travaglini, One-loop gauge theory amplitudes in $N=4$ super Yang-Mills from MHV vertices, Nucl. Phys. B 706 (2005) 150 [hep-th/0407214] [SPIRES].

[51] Z. Bern, L.J. Dixon and D.A. Kosower, All next-to-maximally helicity-violating one-loop gluon amplitudes in $N=4$ super-Yang-Mills theory, Phys. Rev. D 72 (2005) 045014 [hep-th/0412210] [SPIRES].

[52] G.P. Korchemsky and E. Sokatchev, Twistor transform of all tree amplitudes in $N=4$ SYM theory, arXiv: 0907.4107 [SPIRES].

[53] J.M. Drummond, M. Spradlin, A. Volovich and C. Wen, Tree-level amplitudes in $N=8$ supergravity, Phys. Rev. D 79 (2009) 105018 [arXiv:0901.2363] [SPIRES].

[54] N. Arkani-Hamed, F. Cachazo, C. Cheung and J. Kaplan, A duality for the S matrix, arXiv:0907. 5418 [SPIRES].

[55] A. Hodges, Eliminating spurious poles from gauge-theoretic amplitudes, arXiv:0905.1473 [SPIRES].

[56] L. Mason and D. Skinner, Dual superconformal invariance, momentum twistors and Grassmannians, JHEP 11 (2009) 045 [arXiv:0909.0250] [SPIRES].

[57] N. Arkani-Hamed, F. Cachazo and C. Cheung, The Grassmannian origin of dual superconformal invariance, arXiv:0909.0483 [SPIRES].

[58] M. Bullimore, L. Mason and D. Skinner, Twistor-strings, Grassmannians and leading singularities, arXiv:0912.0539 [SPIRES].

[59] J. Kaplan, Unraveling $L_{n, k}$ : Grassmannian kinematics, arXiv:0912.0957 [SPIRES].

[60] J.M. Drummond, J. Henn, G.P. Korchemsky and E. Sokatchev, On planar gluon amplitudes/Wilson loops duality, Nucl. Phys. B 795 (2008) 52 [arXiv:0709.2368] [SPIRES].

[61] L.F. Alday and J.M. Maldacena, Gluon scattering amplitudes at strong coupling, JHEP 06 (2007) 064 [arXiv: 0705.0303] [SPIRES].

[62] H. Kawai, D.C. Lewellen and S.H. Henry Tye, A relation between tree amplitudes of closed and open strings, Nucl. Phys. B 269 (1986) 1 [SPIRES].

[63] H. Elvang and D.Z. Freedman, Note on graviton MHV amplitudes, JHEP 05 (2008) 096 [arXiv:0710.1270] [SPIRES].

[64] L.J. Mason and D. Skinner, An ambitwistor Yang-Mills Lagrangian, Phys. Lett. B 636 (2006) 60 [hep-th/0510262] [SPIRES].

[65] K. Risager, A direct proof of the CSW rules, JHEP 12 (2005) 003 [hep-th/0508206] [SPIRES].

[66] H. Elvang, D.Z. Freedman and M. Kiermaier, Proof of the MHV vertex expansion for all tree amplitudes in $N=4$ SYM theory, JHEP 06 (2009) 068 [arXiv:0811.3624] [SPIRES].

[67] M. Kiermaier and S.G. Naculich, A super MHV vertex expansion for $N=4 S Y M$ theory, JHEP 05 (2009) 072 [arXiv: 0903.0377] [SPIRES].

[68] V.P. Nair, A current algebra for some gauge theory amplitudes, Phys. Lett. B 214 (1988) 215 [SPIRES].

[69] I.M. Gel'fand and G.E. Shilov, Generalised functions, Academic Press, U.S.A. (1964) [ISBN:0122795016]. 
[70] M.B. Green, J.H. Schwarz and L. Brink, $N=4$ Yang-Mills and $N=8$ supergravity as limits of string theories, Nucl. Phys. B 198 (1982) 474 [SPIRES].

[71] Z. Bern, L.J. Dixon, D.C. Dunbar and D.A. Kosower, One-loop n-point gauge theory amplitudes, unitarity and collinear limits, Nucl. Phys. B 425 (1994) 217 [hep-ph/9403226] [SPIRES].

[72] R. Penrose, The universal bracket factor, in Advances in Twistor Theory, Pitman Research Notes in Maths 37, U.S.A. (1979).

[73] R. Penrose and W. Rindler, Spinors and space-time, volume I, Cambridge University Press, Cambridge U.K. (1984).

[74] R. Penrose and W. Rindler, Spinors and space-time, volume II, Cambridge University Press, Cambridge U.K. (1986).

[75] A. Hodges, Elemental states, section 1.5.11 in Further advances in twistor theory, volume I, L.J. Mason and L.P. Hughston eds., Pitman Research Notes in Math. 231, U.S.A. (1986).

[76] M.G. Eastwood, R. Penrose and R.O. Wells, Cohomology and massless fields, Commun. Math. Phys. 78 (1981) 305 [SPIRES].

[77] T.N. Bailey, M.G. Eastwood, R. Gover and L.J. Mason, Complex analysis and the Funk transform, J. Korean Math. Soc. 40 (2003) 577.

[78] W. Fulton and R. Pandharipande, Notes on stable maps and quantum cohomology, in Proc. Sympos. Pure Math. 62 Part 2 (1997) [alg-geom/9608011].

[79] S. Gukov, L. Motl and A. Neitzke, Equivalence of twistor prescriptions for super Yang-Mills, Adv. Theor. Math. Phys. 11 (2007) 199 [hep-th/0404085] [SPIRES].

[80] C. Vergu, On the factorisation of the connected prescription for Yang-Mills amplitudes, Phys. Rev. D 75 (2007) 025028 [hep-th/0612250] [SPIRES].

[81] L. Dolan, C.R. Nappi and E. Witten, Yangian symmetry in $D=4$ superconformal Yang-Mills theory, hep-th/0401243 [SPIRES].

[82] I. Bena, J. Polchinski and R. Roiban, Hidden symmetries of the $A d S_{5} \times S^{5}$ superstring, Phys. Rev. D 69 (2004) 046002 [hep-th/0305116] [SPIRES].

[83] N. Beisert, R. Ricci, A.A. Tseytlin and M. Wolf, Dual superconformal symmetry from $A d S_{5} \times S^{5}$ superstring integrability, Phys. Rev. D 78 (2008) 126004 [arXiv:0807.3228] [SPIRES].

[84] L.J. Mason, Global anti-self-dual Yang-Mills fields in split signature and their scattering, math-ph/0505039 [SPIRES].

[85] L.J. Mason, Global solutions of the self-duality equations in split signature, in Further Advances In twistor Theory, volume II, Pitman Research Notes in Maths 232, U.S.A. (1995), pg. 39 [SPIRES].

[86] G.A. Sparling, Inversion for the radon line transform in higher dimensions, Phil. Trans. Roy. Soc. Ser. A 356 (1998) 3041.

[87] N.M.J. Woodhouse, Contour integrals for the ultra-hyperbolic wave equation, Proc. Roy. Soc. London A 438 (1992) 197.

[88] T.N. Bailey, M.G. Eastwood, R. Gover and L.J. Mason, The Funk transform as a Penrose transform, Math. Proc. Camb. Phil. Soc. 125 (1999) 67. 
[89] T.N. Bailey and M.G. Eastwood, Twistor results for integral transforms, Contemp. Math. 278 (2001) 77.

[90] C. LeBrun and L.J. Mason, Zoll manifolds and complex surfaces, J. Diff. Geom. 61 (2002) 453.

[91] C. LeBrun and L.J. Mason, Nonlinear gravitons, null geodesics and holomorphic disks, math/0504582 [SPIRES].

[92] C. LeBrun and L.J. Mason, The Einstein-Weyl equations, scattering maps and holomorphic disks, arXiv:0806.3761 [SPIRES].

[93] A. Ferber, Supertwistors and conformal supersymmetry, Nucl. Phys. B 132 (1978) 55 [SPIRES].

[94] I.L. Buchbinder and S.M. Kuzenko, Ideas and methods of supersymmetry and supergravity: or a walk through superspace, IOP, Bristol U.K. (1998). 